\title{
A General Strategy for Ligation of Organic and Biological Molecules to Polyoxotungstates
}

\author{
Kevin Micoine, Bernold Hasenknopf, Serge Thorimbert, Emmanuel Lacôte, and Max \\ Malacria
}

\section{Supporting information}

63 pages

General remarks: Reagents and chemicals were purchased from commercial sources and used as received. Functionnalized polyoxometalates $\operatorname{TBA}_{4}\left[\alpha-\mathrm{PW}_{11} \mathrm{O}_{39}\left\{\mathrm{SnCH}_{2} \mathrm{CH}_{2} \mathrm{CO}_{2} \mathrm{H}\right\}\right]$, $\mathrm{TBA}_{6} \mathrm{H}\left[\alpha_{1}-\mathrm{P}_{2} \mathrm{~W}_{17} \mathrm{O}_{61}\left\{\mathrm{SnCH}_{2} \mathrm{CH}_{2} \mathrm{CO}_{2} \mathrm{H}\right\}\right]$, and $\mathrm{TBA}_{6} \mathrm{~K}\left[\alpha_{2}-\mathrm{P}_{2} \mathrm{~W}_{17} \mathrm{O}_{61}\left\{\mathrm{SnCH}_{2} \mathrm{CH}_{2} \mathrm{CO}_{2} \mathrm{H}\right\}\right]$ were prepared as reported in the literature. ${ }^{1}$ Unless otherwise noted, reactions were carried out under air atmosphere with magnetic stirring. $\mathrm{CH}_{3} \mathrm{CN}$ was dried and distilled from $\mathrm{CaH}_{2}$ for amide coupling. Thin-layer chromatography (TLC) was performed on Merck $60 \mathrm{~F}_{254}$ silica gel. Merck Geduran SI $60 \AA$ silica gel $(40-63 \mu \mathrm{m})$ was used for column chromatography. IR spectra were recorded from a Bruker Tensor 27 ATR diamond PIKE spectrophotometer. ${ }^{1} \mathrm{H}$ NMR $\left[{ }^{13} \mathrm{C}\right.$ NMR] spectra were recorded at room temperature with a $400 \mathrm{MHz}[100 \mathrm{MHz}]$ Bruker AVANCE 400 spectrometer or a $200 \mathrm{MHz}$ [50 MHz] Bruker AVANCE 200. Chemical shifts are given in ppm, referenced to the residual resonances of the solvents $(\delta=1.94$ or 118.3 , for $\left.\mathrm{CD}_{3} \mathrm{CN}\right)$. Coupling constants $\left(\mathcal{J}\right.$ ) are given in Hertz $(\mathrm{Hz}) .{ }^{31} \mathrm{P}$ NMR spectra were obtained at $298 \mathrm{~K}$ in $5 \mathrm{~mm}$ o.d. tube at $162 \mathrm{MHz}$, by use of a Bruker AVANCE 400 at a concentration of $100 \mathrm{mg} / 0.5 \mathrm{~mL}$. External $85 \% \mathrm{H}_{3} \mathrm{PO}_{4}$ in coaxial tube was used as reference. Mass spectrometry experiments have been carried out on an electrospray-ion trap instrument (Bruker, Esquire 3000). The $50 \mathrm{pmol} \cdot \mu \mathrm{L}^{-1}$ solutions of POMs were infused using a syringe pump $\left(160 \mu \mathrm{L} \cdot \min ^{-1}\right)$. The negative ion mode was used with capillary high voltage $3500 \mathrm{~V}$. The orifice/skimmer voltage difference was set to $45 \mathrm{~V}$ to avoid decomposition of the POMs. The low-mass-cutoff (LMCO) of the ion trap was set to $80 \mathrm{Th}$. $(\mathrm{K}),\left(\alpha_{1}\right),\left(\alpha_{2}\right)$ represent respectively the formulas of lacunary Keggin polyanion $\left(\left[\mathrm{PW}_{11} \mathrm{O}_{39}\right]^{7-}\right)$, lacunary Dawson polyanions $\left(\left[\mathrm{P}_{2} \mathrm{~W}_{17} \mathrm{O}_{61}\right]^{10-}, \alpha_{1}\right.$ and $\alpha_{2}$ isomers). Elemental analyses were carried out by the "Service Central d'Analyse", CNRS, Vernaison, France or by the ICSN, CNRS, Gif, France.

\section{General procedure for coupling to Dawson polyoxometalates (GPA).}

To a solution of $\mathrm{TBA}_{5}\left[\mathrm{Et}_{3} \mathrm{NH}^{+}\right]_{2}\left[\mathrm{P}_{2} \mathrm{~W}_{17} \mathrm{O}_{61}\left\{\mathrm{SnCH}_{2} \mathrm{CH}_{2} \mathrm{C}(=\mathrm{O}) \mathrm{NHCH}_{2} \mathrm{C} \equiv \mathrm{CH}\right\}\right]$ (1 or 3), or $\mathrm{TBA}_{5}\left[\mathrm{Et}_{3} \mathrm{NH}^{+}\right]_{2}\left[\mathrm{P}_{2} \mathrm{~W}_{17} \mathrm{O}_{61}\left\{\mathrm{SnCH}_{2} \mathrm{CH}_{2} \mathrm{C}(=\mathrm{O}) \mathrm{NH}\left(\mathrm{CH}_{2}\right)_{3} \mathrm{~N}_{3}\right\}\right]$ (2 or $4,0.026 \mathrm{mmol}, 150 \mathrm{mg}$ ) in acetonitrile $(1 \mathrm{~mL})$ was added the azide or the alkyne $(0.052 \mathrm{mmol}, 2$ equiv). A solution of $\mathrm{CuSO}_{4} \cdot 5 \mathrm{H}_{2} \mathrm{O}(0.026 \mathrm{mmol}, 1$ equiv, $6.5 \mathrm{mg})$ in water $(1 \mathrm{~mL})$ and a solution of sodium ascorbate $(0.26 \mathrm{mmol}, 10$ equiv, $52 \mathrm{mg})$ in water $(1 \mathrm{~mL})$ were then added. The mixture was stirred at room temp for 24 hours. A cation-exchange resin (Amberlyst 15, 16-50 mesh, TBA ${ }^{+}$ form) was added, followed by acetone $(10 \mathrm{~mL})$ and the mixture was stirred for 1 hour or until disappearance of the precipitate (if any appeared). The resin was filtered off and the filtrate was concentrated in vacuo. Dichloromethane $(30 \mathrm{~mL})$, and water $(30 \mathrm{~mL})$ were added to the crude residue. The organic phase was washed with water $(30 \mathrm{~mL})$ and concentrated in vacuo to deliver a white solid. This solid was dissolved in acetone $(2 \mathrm{~mL})$, and precipitated upon addition of $\mathrm{EtOH} / \mathrm{Et}_{2} \mathrm{O}(2 \mathrm{~mL} / 30 \mathrm{~mL})$. The solid was isolated by centrifugation, washed with $\mathrm{Et}_{2} \mathrm{O}$ and dried in vacuo to afford the desired POM.

\footnotetext{
${ }^{1}$ S. Bareyt, S. Piligkos, B. Hasenknopf, P. Gouzerh, E. Lacôte, S. Thorimbert, M. Malacria, J. Am. Chem. Soc. 2005, 127, 6788-6794.
} 
General procedure for couplings to Keggin polyoxometalates (GPB).

To a solution of $\mathrm{TBA}_{3}\left[\mathrm{Et}_{3} \mathrm{NH}^{+}\right]\left[\mathrm{PW}_{11} \mathrm{O}_{39}\left\{\mathrm{SnCH}_{2} \mathrm{CH}_{2} \mathrm{C}(=\mathrm{O}) \mathrm{NHCH}_{2} \mathrm{C} \equiv \mathrm{CH}\right\}\right]$ (5) or $\mathrm{TBA}_{3}\left[\mathrm{Et}_{3} \mathrm{NH}^{+}\right]\left[\mathrm{PW}_{11} \mathrm{O}_{39}\left\{\mathrm{SnCH}_{2} \mathrm{CH}_{2} \mathrm{C}(=\mathrm{O}) \mathrm{NH}\left(\mathrm{CH}_{2}\right)_{3} \mathrm{~N}_{3}\right\}\right](6)(0.04 \mathrm{mmol}, 150 \mathrm{mg})$ in acetonitrile $(1 \mathrm{~mL})$ was added the azide or the alkyne $\left(0.08 \mathrm{mmol}, 2\right.$ equiv). A solution of $\mathrm{CuSO}_{4} \cdot 5 \mathrm{H}_{2} \mathrm{O}$ $(0.04 \mathrm{mmol}, 1$ equiv, $10 \mathrm{mg})$ in water $(1 \mathrm{~mL})$ and a solution of sodium ascorbate $(1.6 \mathrm{mmol}$, 40 equiv, $316 \mathrm{mg}$ ) in water $(1 \mathrm{~mL})$ were then added. The mixture was stirred at room temp for 24 hours. A cation-exchange resin (Amberlyst 15, 16-50 mesh, TBA ${ }^{+}$form) was added, followed by acetone $(10 \mathrm{~mL})$ and the mixture was stirred for 1 hour or until disappearance of the precipitate (if any appeared). The resin was filtered off and the filtrate was concentrated in vacuo. The white powder residue was washed twice with water $(10 \mathrm{~mL})$ and isolated by centrifugation. After drying in vacuo, it was dissolved in acetone $(2 \mathrm{~mL})$ and precipitated by adding $\mathrm{CH}_{2} \mathrm{Cl}_{2} / \mathrm{Et}_{2} \mathrm{O}(2 \mathrm{~mL} / 30 \mathrm{~mL})$. The new solid was isolated by centrifugation, washed with $\mathrm{Et}_{2} \mathrm{O}$ and dried in vacuo to afford the desired POM.

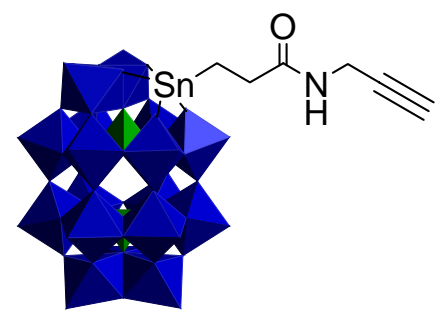

$\mathbf{T B A}_{5}\left[\mathbf{E t}_{3} \mathbf{N H}^{+}\right]_{2}\left[\left(\boldsymbol{\alpha}_{2}\right) \mathbf{S n C H}_{2} \mathbf{C H}_{2} \mathbf{C}(=\mathbf{O}) \mathbf{N H C H}_{2} \mathbf{C} \equiv \mathbf{C H}\right]$. Following our previously reported procedure, ${ }^{1} 1$ was obtained in 85\% yield. White solid. IR: $\tilde{v}=2961(\mathrm{~m}), 2934(\mathrm{w}), 2872(\mathrm{~m}), 1639$ (w), 1483 (m), 1379 (w), 1088 (s), 947 (s), 901 (s), 776 (vs) cm ${ }^{-1}$; ${ }^{1} \mathrm{H}$ NMR (400 MHz, CD ${ }_{3} \mathrm{CN}$ ): $\delta 0.98\left(\mathrm{t}, J=7.1 \mathrm{~Hz}, 60 \mathrm{H}, \mathrm{N}\left[\left(\mathrm{CH}_{2}\right)_{3} M e\right]_{4}\right), 1.10\left(\mathrm{t}, J=8.0 \mathrm{~Hz}, 2 \mathrm{H}, \mathrm{SnCH}_{2}\right), 1.38-1.44(\mathrm{~m}, 58 \mathrm{H}$, $\left.\mathrm{N}\left(\mathrm{CH}_{2} \mathrm{CH}_{2} \mathrm{CH}_{2} \mathrm{Me}\right)_{4}+\mathrm{HN}\left(\mathrm{CH}_{2} \mathrm{Me}\right)_{3}\right), 1.55-1.70\left(\mathrm{~m}, 40 \mathrm{H}, \mathrm{N}\left(\mathrm{CH}_{2} \mathrm{CH}_{2} \mathrm{CH}_{2} \mathrm{Me}\right)_{4}\right), 2.40$ (t, $J=2.4 \mathrm{~Hz}$, $1 \mathrm{H}, \mathrm{C} \equiv \mathrm{CH}), 2.50\left(\mathrm{t}, J=8.0 \mathrm{~Hz}, 2 \mathrm{H}, \mathrm{CH}_{2} \mathrm{C}=\mathrm{O}\right), 3.08-3.25\left(\mathrm{~m}, 40 \mathrm{H}, \mathrm{N}\left(\mathrm{CH}_{2} \mathrm{Pr}\right)_{4}\right), 3.35-3.52(\mathrm{~m}, 12 \mathrm{H}$, $\left.\mathrm{HN}\left(\mathrm{CH}_{2} \mathrm{CH}_{3}\right)_{3}\right), 3.95\left(\mathrm{dd}, J=5.6,2.4 \mathrm{~Hz}, 2 \mathrm{H}, \mathrm{CONHCH}_{2}\right), 7.02(\mathrm{t}, J=6 \mathrm{~Hz}, 1 \mathrm{H}, \mathrm{NH}) ;{ }^{13} \mathrm{C} \mathrm{NMR}$

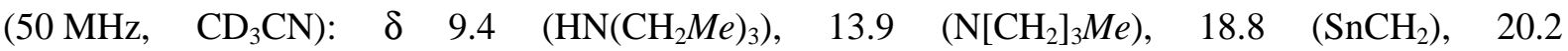
$\left(\mathrm{N}\left(\mathrm{CH}_{2} \mathrm{CH}_{2} \mathrm{CH}_{2} \mathrm{Me}\right)_{4}\right), \quad 24.2 \quad\left(\mathrm{~N}\left(\mathrm{CH}_{2} \mathrm{CH}_{2} \mathrm{CH}_{2} \mathrm{Me}\right)_{4}\right), \quad 29.2 \quad\left(\mathrm{NHCH}_{2}\right), \quad 31.8 \quad\left(\mathrm{CH}_{2} \mathrm{C}=\mathrm{O}\right), \quad 47.9$ $\left(\mathrm{HN}\left(\mathrm{CH}_{2} \mathrm{CH}_{3}\right)_{3}\right), 58.9\left(\mathrm{~N}\left(\mathrm{CH}_{2} \mathrm{Pr}\right)_{4}\right), 71.4\left(\mathrm{CH}_{2} \mathrm{C} \equiv \mathrm{CH}\right), 81.6\left(\mathrm{CH}_{2} \mathrm{C} \equiv \mathrm{CH}\right), 175.1(\mathrm{C}=\mathrm{O}) ;{ }^{31} \mathrm{P}$ NMR $\left(162 \mathrm{MHz}, \mathrm{CD}_{3} \mathrm{CN}\right): \delta-9.3\left(\mathrm{~s}+\mathrm{d}, 1 \mathrm{P}, J_{S n P}=25.9 \mathrm{~Hz}\right),-12.6(\mathrm{~s}, 1 \mathrm{P})$.

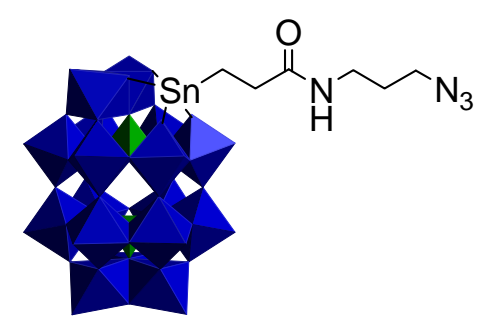

$\mathbf{T B A}_{5}\left[\mathbf{E t}_{3} \mathbf{N H}^{+}\right]_{2}\left[\left(\boldsymbol{\alpha}_{2}\right) \mathbf{S n C H}_{2} \mathbf{C H}_{2} \mathbf{C}(=\mathbf{O}) \mathbf{N H}\left(\mathrm{CH}_{2}\right)_{3} \mathbf{N}_{3}\right]$. Following our previously reported procedure, ${ }^{1}$ 2 was obtained in 88\% yield. IR: $\tilde{\boldsymbol{v}}=2961(\mathrm{~m}), 2934(\mathrm{w}), 2873(\mathrm{~m}), 2095(\mathrm{~m}), 1634(\mathrm{w}), 1482(\mathrm{~m})$, 1379 (w), 1086 (s), 945 (s), 898 (s), 764 (vs) cm ${ }^{-1} ;{ }^{1} \mathrm{H} \mathrm{NMR} \mathrm{(400} \mathrm{MHz,} \mathrm{CD}_{3} \mathrm{CN}$ ): $\delta 0.98$ (t, $J=7.1 \mathrm{~Hz}$, $\left.60 \mathrm{H}, \mathrm{N}\left[\left(\mathrm{CH}_{2}\right)_{3} \mathrm{Me}\right]_{4}\right), 1.11\left(\mathrm{t}, J=8.0 \mathrm{~Hz}, 2 \mathrm{H}, \mathrm{SnCH}_{2}\right), 1.38-1.44\left(\mathrm{~m}, 58 \mathrm{H}, \mathrm{N}\left(\mathrm{CH}_{2} \mathrm{CH}_{2} \mathrm{CH}_{2} \mathrm{Me}\right)_{4}+\right.$ $\left.\mathrm{HN}\left(\mathrm{CH}_{2} \mathrm{CH}_{3}\right)_{3}\right), 1.55-1.70\left(\mathrm{~m}, 40 \mathrm{H}, \mathrm{N}\left(\mathrm{CH}_{2} \mathrm{CH}_{2} \mathrm{CH}_{2} \mathrm{Me}\right)_{4}\right), 1.75-1.79$ (m, $\left.2 \mathrm{H}, \mathrm{CH}_{2} \mathrm{CH}_{2} \mathrm{CH}_{2}\right), 2.49$ (t, $\left.J=8.0 \mathrm{~Hz}, 2 \mathrm{H}, \mathrm{CH}_{2} \mathrm{C}=\mathrm{O}\right), 3.08-3.25\left(\mathrm{~m}, 42 \mathrm{H}, \mathrm{N}\left(\mathrm{CH}_{2} \mathrm{CH}_{2} \mathrm{CH}_{2} \mathrm{Me}\right)_{4}+\mathrm{NHCH}_{2}\right), 3.35-3.52(\mathrm{~m}, 14 \mathrm{H}$, $\left.\mathrm{HN}\left(\mathrm{CH}_{2} \mathrm{CH}_{3}\right)_{3}+\mathrm{CH}_{2} \mathrm{~N}_{3}\right), 6.90(\mathrm{t}, J=6 \mathrm{~Hz}, 1 \mathrm{H}, \mathrm{NH}) ;{ }^{13} \mathrm{C} \mathrm{NMR}\left(50 \mathrm{MHz}, \mathrm{CD}_{3} \mathrm{CN}+5 \% \mathrm{D}_{2} \mathrm{O}\right.$;

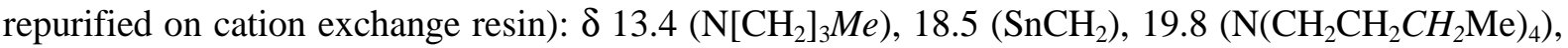
$23.8\left(\mathrm{~N}\left(\mathrm{CH}_{2} \mathrm{CH}_{2} \mathrm{CH}_{2} \mathrm{Me}\right)_{4}\right), 28.6\left(\mathrm{CH}_{2} \mathrm{CH}_{2} \mathrm{CH}_{2}\right), 31.3\left(\mathrm{CH}_{2} \mathrm{C}=\mathrm{O}\right), 36.9\left(\mathrm{NHCH}_{2}\right), 49.3\left(\mathrm{CH}_{2} \mathrm{~N}_{3}\right), 58.6$ $\left(\mathrm{N}\left(\mathrm{CH}_{2} \mathrm{Pr}\right)_{4}\right), 176.1(\mathrm{C}=\mathrm{O}) ;{ }^{31} \mathrm{P}$ NMR $\left(162 \mathrm{MHz}, \mathrm{CD}_{3} \mathrm{CN}\right): \delta-9.2\left(\mathrm{~s}+\mathrm{d}, 1 \mathrm{P}, J_{S n P}=22.7 \mathrm{~Hz}\right),-12.6(\mathrm{~s}$, $1 \mathrm{P})$. 


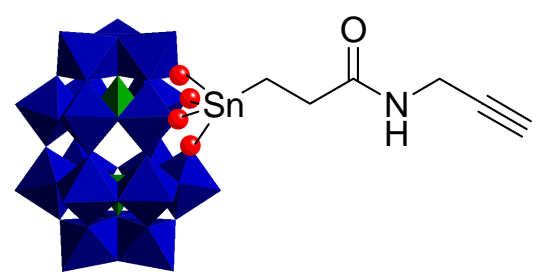

$\mathbf{T B A}_{5}\left[\mathbf{E t}_{3} \mathbf{N H}^{+}\right]_{2}\left[\left(\boldsymbol{\alpha}_{1}\right) \mathbf{S n C H}_{2} \mathbf{C H}_{2} \mathbf{C}(=\mathbf{O}) \mathbf{N H C H}_{2} \mathbf{C} \equiv \mathbf{C H}\right]$. Following our previously reported procedure, ${ }^{1} \mathbf{3}$ was obtained in $80 \%$ yield. IR: $\tilde{\boldsymbol{v}}=2961(\mathrm{~m}), 2934(\mathrm{w}), 2873(\mathrm{~m}), 1638(\mathrm{w}), 1465(\mathrm{~m})$, 1380 (w), 1082 (s), 946 (s), 904 (s), 771(vs) cm ${ }^{-1} ;{ }^{1} \mathrm{H}$ NMR (400 MHz, $\mathrm{CD}_{3} \mathrm{CN}$ ): $\delta 0.98$ (t, J=7.1 Hz, $\left.60 \mathrm{H}, \mathrm{N}\left(\mathrm{CH}_{2} \mathrm{CH}_{2} \mathrm{CH}_{2} \mathrm{Me}\right)_{4}\right), 1.04-1.10\left(\mathrm{~m}, 2 \mathrm{H}, \mathrm{SnCH}_{2}\right), 1.38-1.44\left(\mathrm{~m}, 58 \mathrm{H}, \mathrm{N}\left(\mathrm{CH}_{2} \mathrm{CH}_{2} \mathrm{CH}_{2} \mathrm{Me}\right)_{4}+\right.$ $\left.\mathrm{HN}\left(\mathrm{CH}_{2} \mathrm{Me}\right)_{3}\right), 1.55-1.70\left(\mathrm{~m}, 40 \mathrm{H}, \mathrm{N}\left(\mathrm{CH}_{2} \mathrm{CH}_{2} \mathrm{CH}_{2} \mathrm{Me}\right)_{4}\right), 2.36(\mathrm{t}, J=2.4 \mathrm{~Hz}, 1 \mathrm{H}, \mathrm{C} \equiv \mathrm{CH}), 2.56-2.72$ $\left(\mathrm{m}, 2 \mathrm{H}, \mathrm{CH}_{2} \mathrm{C}=\mathrm{O}\right), 3.08-3.25\left(\mathrm{~m}, 40 \mathrm{H}, \mathrm{N}\left(\mathrm{CH}_{2} \mathrm{CH}_{2} \mathrm{CH}_{2} \mathrm{Me}\right)_{4}\right), 3.35-3.52\left(\mathrm{~m}, 12 \mathrm{H}, \mathrm{HN}\left(\mathrm{CH}_{2} \mathrm{Me}\right)_{3}\right)$, 4.02 (bs, $\left.2 \mathrm{H}, \mathrm{NHCH}_{2}\right), 7.17$ (t, $\left.J=5.8 \mathrm{~Hz}, 1 \mathrm{H}, \mathrm{NH}\right) ;{ }^{31} \mathrm{P} \mathrm{NMR}\left(162 \mathrm{MHz}, \mathrm{CD}_{3} \mathrm{CN}\right): \delta-6.6(\mathrm{~s}+\mathrm{d}, 1 \mathrm{P}$, $\left.J_{S n P}=40.5 \mathrm{~Hz}\right),-12.1(\mathrm{~s}, 1 \mathrm{P})$.

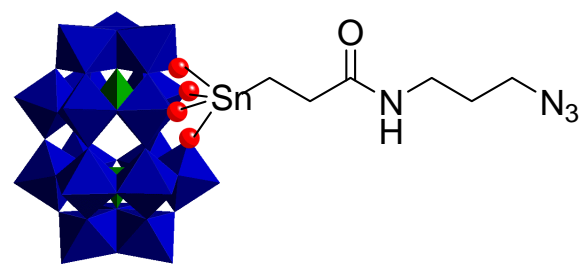

$\mathbf{T B A}_{4} \mathbf{H}\left[\mathbf{E t}_{3} \mathbf{N H}^{+}\right]_{2}\left[\left(\boldsymbol{\alpha}_{1}\right) \mathbf{S n C H}_{2} \mathbf{C H}_{2} \mathbf{C}(=\mathbf{O}) \mathbf{N H}\left(\mathbf{C H}_{2}\right)_{3} \mathbf{N}_{3}\right]$. Following our previously reported procedure, ${ }^{1} 4$ was obtained in $80 \%$ yield. IR: $\tilde{\boldsymbol{v}}=2962(\mathrm{~m}), 2934(\mathrm{w}), 2873(\mathrm{~m}), 2096(\mathrm{~m}), 1631(\mathrm{w})$, 1483 (m), 1380 (w), 1084 (s), 949 (s), 907 (s), 776 (vs) cm ${ }^{-1} ;{ }^{1} \mathrm{H}$ NMR (400 MHz, CD ${ }_{3} \mathrm{CN}$ ): $\delta 0.98$ (t, $\left.J=7.1 \mathrm{~Hz}, \quad 48 \mathrm{H}, \quad \mathrm{N}\left(\mathrm{CH}_{2} \mathrm{CH}_{2} \mathrm{CH}_{2} \mathrm{Me}\right)_{4}\right), \quad 1.04-1.10 \quad\left(\mathrm{~m}, 2 \mathrm{H}, \quad \mathrm{SnCH}_{2}\right), \quad 1.38-1.44 \quad(\mathrm{~m}, 50 \mathrm{H}$, $\left.\mathrm{N}\left(\mathrm{CH}_{2} \mathrm{CH}_{2} \mathrm{CH}_{2} \mathrm{Me}\right)_{4}+\mathrm{HN}\left(\mathrm{CH}_{2} \mathrm{CH}_{3}\right)_{3}\right), 1.55-1.70$ (m, $\left.32 \mathrm{H}, \mathrm{N}\left(\mathrm{CH}_{2} \mathrm{CH}_{2} \mathrm{CH}_{2} \mathrm{Me}\right)_{4}\right), 1.81$ (quint., $\left.J=6.6 \mathrm{~Hz}, 2 \mathrm{H}, \mathrm{CH}_{2} \mathrm{CH}_{2} \mathrm{CH}_{2}\right), 2.64\left(\mathrm{t}, J=7.6 \mathrm{~Hz}, 2 \mathrm{H}, \mathrm{CH}_{2} \mathrm{C}=\mathrm{O}\right), 3.08-3.25\left(\mathrm{~m}, 34 \mathrm{H}, \mathrm{N}\left(\mathrm{CH}_{2} \mathrm{Pr}\right)_{4}+\right.$ $\left.\mathrm{NHCH}_{2}\right), 3.27-3.33\left(\mathrm{~m}, 2 \mathrm{H}, \mathrm{CH}_{2} \mathrm{~N}_{3}\right), 3.35-3.52\left(\mathrm{~m}, 12 \mathrm{H}, \mathrm{HN}\left(\mathrm{CH}_{2} \mathrm{Me}\right)_{3}\right), 7.04$ (bs, $\left.1 \mathrm{H}, \mathrm{NH}\right) ;{ }^{31} \mathrm{P}$ NMR $\left(162 \mathrm{MHz}, \mathrm{CD}_{3} \mathrm{CN}\right): \delta-6.6\left(\mathrm{~s}+\mathrm{d}, 1 \mathrm{P}, J_{S n P}=38.9 \mathrm{~Hz}\right),-12.1(\mathrm{~s}, 1 \mathrm{P})$.

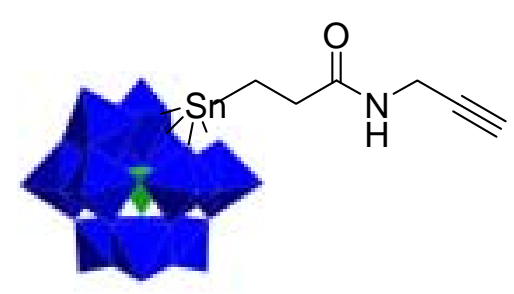

$\mathbf{T B A}_{3}\left(\mathbf{E t}_{3} \mathbf{N H}^{+}\right)\left[(\mathbf{K}) \mathbf{S n C H}_{2} \mathbf{C H}_{2} \mathbf{C}(=\mathbf{O}) \mathbf{N H C H}_{2} \mathbf{C} \equiv \mathbf{C H}\right]$. Following our previously reported procedure, ${ }^{1}$ 5 was obtained quantitatively. IR: $\tilde{\boldsymbol{v}}=2960(\mathrm{~m}), 2934(\mathrm{w}), 2873(\mathrm{~m}), 1670(\mathrm{w}), 1483(\mathrm{~m}), 1380(\mathrm{w})$, 1064 (s), 959 (s), 881 (s), 792 (vs) cm ${ }^{-1} ;{ }^{1} \mathrm{H}$ NMR (400 MHz, CD ${ }_{3} \mathrm{CN}$ ): $\delta 0.99$ (t, $J=7.1 \mathrm{~Hz}, 38 \mathrm{H}$, $\left.\mathrm{N}\left(\mathrm{CH}_{2} \mathrm{CH}_{2} \mathrm{CH}_{2} \mathrm{Me}\right)_{4}+\mathrm{SnCH}_{2}\right), 1.38-1.44\left(\mathrm{~m}, 33 \mathrm{H}, \mathrm{N}\left(\mathrm{CH}_{2} \mathrm{CH}_{2} \mathrm{CH}_{2} \mathrm{Me}\right)_{4}+\mathrm{HN}\left(\mathrm{CH}_{2} \mathrm{Me}\right)_{3}\right), 1.55-1.70$ $\left(\mathrm{m}, 24 \mathrm{H}, \mathrm{N}\left(\mathrm{CH}_{2} \mathrm{CH}_{2} \mathrm{CH}_{2} \mathrm{Me}\right)_{4}\right), 2.40(\mathrm{t}, J=2.4 \mathrm{~Hz}, 1 \mathrm{H}, \mathrm{C} \equiv \mathrm{CH}), 2.53\left(\mathrm{t}, J=8.3 \mathrm{~Hz}, 2 \mathrm{H}, \mathrm{CH}_{2} \mathrm{C}=\mathrm{O}\right)$, 3.08-3.25 (m, $\left.30 \mathrm{H}, \mathrm{N}\left(\mathrm{CH}_{2} \mathrm{Pr}\right)_{4}+\mathrm{HN}\left(\mathrm{CH}_{2} \mathrm{CH}_{3}\right)_{3}\right), 3.95$ (dd, $\left.J=5.6,2.4 \mathrm{~Hz}, 2 \mathrm{H}, \mathrm{NHCH}_{2}\right), 6.84$ (bs,

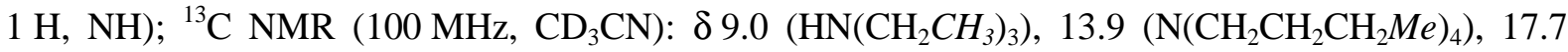

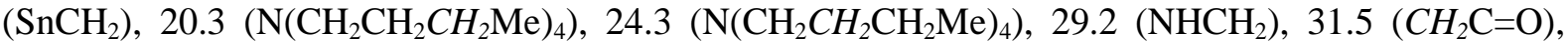
$47.0\left(\mathrm{HN}\left(\mathrm{CH}_{2} \mathrm{CH}_{3}\right)_{3}\right), 59.1\left(\mathrm{~N}\left(\mathrm{CH}_{2} \mathrm{Pr}\right)_{4}\right), 71.5(\mathrm{C} \equiv \mathrm{CH}), 81.6(\mathrm{C} \equiv \mathrm{CH}), 174.0(\mathrm{C}=\mathrm{O}) ;{ }^{31} \mathrm{P} \mathrm{NMR}$ $\left(162 \mathrm{MHz}, \mathrm{CD}_{3} \mathrm{CN}\right): \delta-10.9\left(\mathrm{~s}+\mathrm{d}, 1 \mathrm{P}, J_{S n P}=18.9 \mathrm{~Hz}\right)$. 


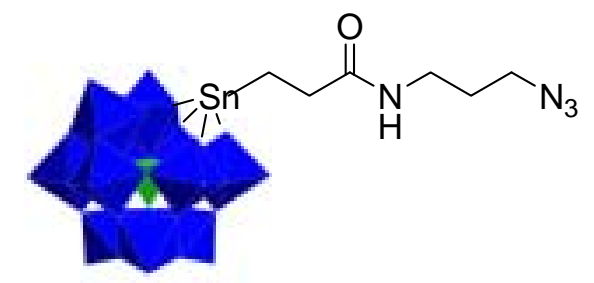

$\mathrm{TBA}_{4}\left[(\mathbf{K}) \mathrm{SnCH}_{2} \mathrm{CH}_{2} \mathrm{C}(=\mathbf{O}) \mathbf{N H}\left(\mathrm{CH}_{2}\right)_{3} \mathbf{N}_{3}\right]$. Following our previously reported procedure, ${ }^{1} \mathbf{6}$ was obtained quantitatively. IR: $\tilde{\boldsymbol{v}}=$ 2960(m), $2934(\mathrm{w}), 2873(\mathrm{~m}), 2095(\mathrm{~m}), 1724(\mathrm{w}), 1662(\mathrm{w}), 1482$ (m), 1380 (w), 1064 (s), 959 (s), 881 (s), 790 (vs) cm ${ }^{-1}$; ${ }^{1} \mathrm{H}$ NMR $\left(400 \mathrm{MHz}, \mathrm{CD}_{3} \mathrm{CN}\right): \delta 0.98$ (t, $\left.J=7.1 \mathrm{~Hz}, 48 \mathrm{H}, \mathrm{N}\left(\mathrm{CH}_{2} \mathrm{CH}_{2} \mathrm{CH}_{2} \mathrm{Me}\right)_{4}\right), 1.38-1.44\left(\mathrm{~m}, 34 \mathrm{H}, \mathrm{N}\left(\mathrm{CH}_{2} \mathrm{CH}_{2} \mathrm{CH}_{2} \mathrm{Me}\right)_{4}+\mathrm{SnCH}_{2}\right), 1.55-1.75$ (m, $\left.34 \mathrm{H}, \mathrm{N}\left(\mathrm{CH}_{2} \mathrm{CH}_{2} \mathrm{CH}_{2} \mathrm{Me}\right)_{4}+\mathrm{CH}_{2} \mathrm{CH}_{2} \mathrm{CH}_{2}\right), 2.48\left(\mathrm{t}, J=8.2 \mathrm{~Hz}, 2 \mathrm{H}, \mathrm{CH}_{2} \mathrm{C}=\mathrm{O}\right), 3.08-3.25(\mathrm{~m}$, $\left.34 \mathrm{H}, \mathrm{N}\left(\mathrm{CH}_{2} \mathrm{CH}_{2} \mathrm{CH}_{2} \mathrm{Me}\right)_{4}+\mathrm{NHCH}_{2}\right), 3.37\left(\mathrm{t}, J=6.8 \mathrm{~Hz}, 2 \mathrm{H}, \mathrm{CH}_{2} \mathrm{~N}_{3}\right), 6.68(\mathrm{~s}, 1 \mathrm{H}, \mathrm{NH}) ;{ }^{31} \mathrm{P} \mathrm{NMR}$ $\left(162 \mathrm{MHz}, \mathrm{CD}_{3} \mathrm{CN}\right): \delta-10.9\left(\mathrm{~s}+\mathrm{d}, 1 \mathrm{P}, J_{S n P}=19.4 \mathrm{~Hz}\right)$. 


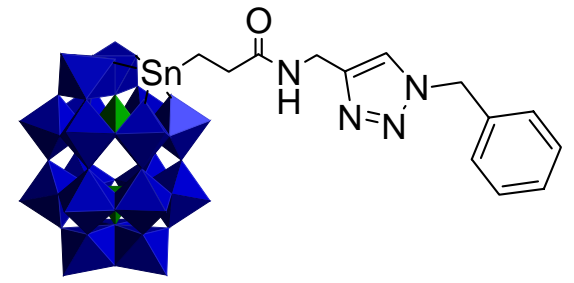

7: $\mathbf{T B A}_{6} \mathbf{H}\left[\left(\boldsymbol{\alpha}_{2}\right) \operatorname{Sn}\left(\mathbf{C H}_{2}\right)_{2} \mathbf{C O N H C H}_{2}\left(\mathbf{C}_{2} \mathbf{H N}_{3}\right)\right.$ Bn]. Following GPA, $\mathbf{7}$ was isolated as a white powder (149 mg, $92 \%)$. IR: $\tilde{v}=2961(\mathrm{~m}), 2934(\mathrm{w}), 2872(\mathrm{~m}), 1663(\mathrm{w}), 1483(\mathrm{~m}), 1379(\mathrm{w}), 1089(\mathrm{~s}), 951$ (s), 900 (s), 776 (vs) cm ${ }^{-1} ;{ }^{1} \mathrm{H}$ NMR $\left(400 \mathrm{MHz}, \mathrm{CD}_{3} \mathrm{CN}\right): \delta 0.98 \quad(\mathrm{t}, J=7.1 \mathrm{~Hz}, \quad 74 \mathrm{H}$, $\left.\mathrm{N}\left(\mathrm{CH}_{2} \mathrm{CH}_{2} \mathrm{CH}_{2} \mathrm{Me}\right)_{4}+\mathrm{SnCH}_{2}\right), 1.38-1.44\left(\mathrm{~m}, 48 \mathrm{H}, \mathrm{N}\left(\mathrm{CH}_{2} \mathrm{CH}_{2} \mathrm{CH}_{2} \mathrm{Me}\right)_{4}\right), 1.55-1.70$ (m, $48 \mathrm{H}$, $\left.\mathrm{N}\left(\mathrm{CH}_{2} \mathrm{CH}_{2} \mathrm{CH}_{2} \mathrm{Me}\right)_{4}\right), 2.52$ (bs, $\left.2 \mathrm{H}, \mathrm{CH}_{2} \mathrm{C}=\mathrm{O}\right), 3.08-3.25$ (m, $\left.48 \mathrm{H}, \mathrm{N}\left(\mathrm{CH}_{2} \mathrm{Pr}\right)_{4}\right), 4.39$ (bs, $2 \mathrm{H}$, $\left.\mathrm{NHCH}_{2}\right), 5.62\left(\mathrm{~s}, 2 \mathrm{H}, \mathrm{NCH}_{2} \mathrm{Ph}\right), 7.24-7.48(\mathrm{~m}, 5 \mathrm{H}$, arom.), 7.84 (bs, $1 \mathrm{H}, \mathrm{NH}), 8.19$ (s, $1 \mathrm{H}$,

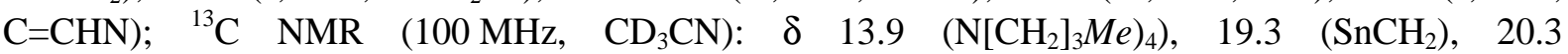
$\left(\mathrm{N}\left(\mathrm{CH}_{2} \mathrm{CH}_{2} \mathrm{CH}_{2} \mathrm{Me}\right)_{4}\right), 24.3\left(\mathrm{~N}\left(\mathrm{CH}_{2} \mathrm{CH}_{2} \mathrm{CH}_{2} \mathrm{Me}\right)_{4}\right), 32.5\left(\mathrm{CH}_{2} \mathrm{C}=\mathrm{O}\right), 36.3\left(\mathrm{NHCH}_{2}\right), 54.2\left(\mathrm{NCH}_{2} \mathrm{Ph}\right)$, $59.1\left(\mathrm{~N}\left(\mathrm{CH}_{2} \mathrm{CH}_{2} \mathrm{CH}_{2} \mathrm{Me}\right)_{4}\right), 124.5(\mathrm{C}=\mathrm{CHN}), 128.8$ (arom.), 129.5 (arom.), 129.7 (arom.), 137.4 (arom.), $147.0 \quad(\mathrm{C}=\mathrm{CHN}), 175.0 \quad(\mathrm{C}=\mathrm{O}) ;{ }^{31} \mathrm{P} \quad \mathrm{NMR} \quad\left(162 \mathrm{MHz}, \mathrm{CD}_{3} \mathrm{CN}\right): \delta-9.44 \quad(\mathrm{~s}+\mathrm{d}, \quad 1 \mathrm{P}$, $\left.\left.J_{S n P}=27.7 \mathrm{~Hz}\right),-12.64-\mathrm{s}, 1 \mathrm{P}\right)$; elemental analysis $(\%)$ for $\mathrm{C}_{109} \mathrm{H}_{232} \mathrm{~N}_{10} \mathrm{O}_{62} \mathrm{P}_{2} \mathrm{SnW}_{17}\left(5981.17 \mathrm{~g} \cdot \mathrm{mol}^{-1}\right)$ : calc. C 21.89, H 3.91, N 2.34; found C 21.57, H 3.66, N 2.21; ESI/MS: see below for full details.

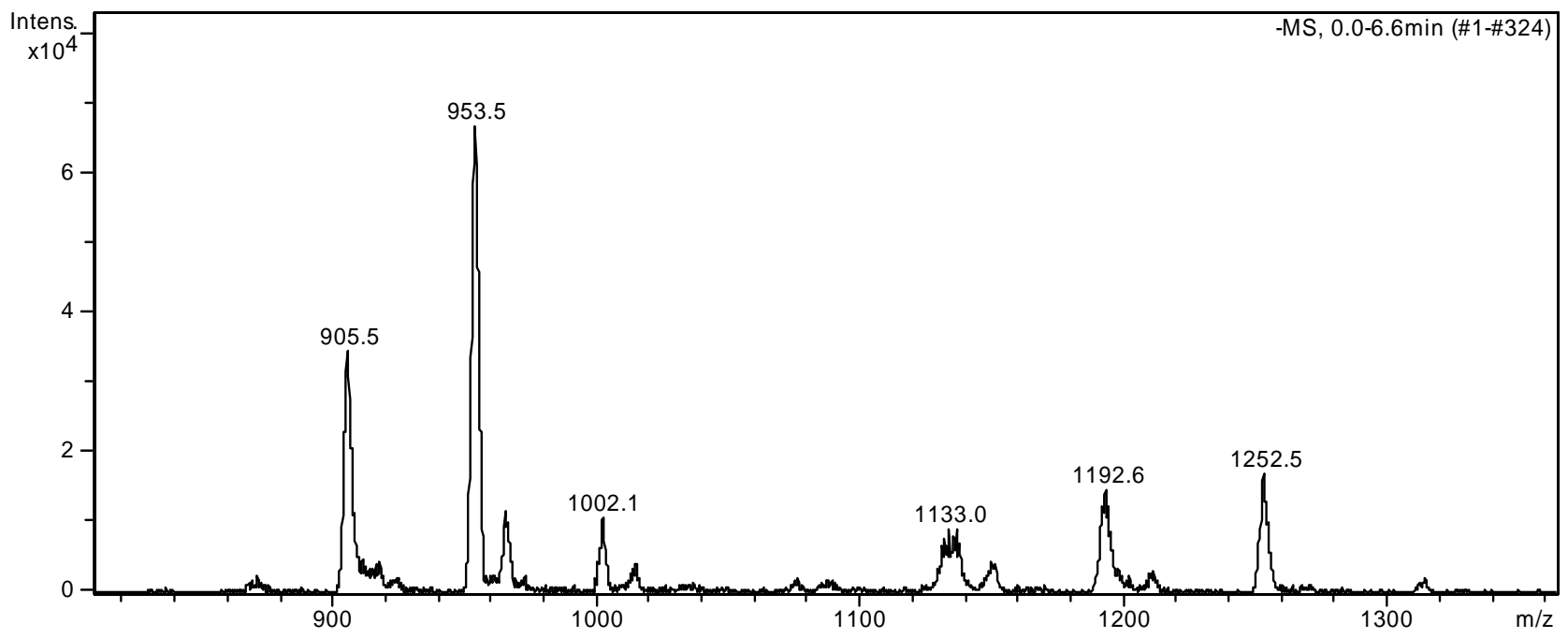

\begin{tabular}{cccccc}
\hline Entry & Charge & $\begin{array}{c}\text { Simulated } \\
\mathrm{m} / \mathrm{z}\end{array}$ & $\begin{array}{c}\text { Observed } \\
\mathrm{m} / \mathrm{z}\end{array}$ & $\begin{array}{c}\text { Relative } \\
\text { intensity }\end{array}$ & Composition \\
\hline 1 & $4-$ & 1252.9 & 1252.5 & 24.2 & $\mathrm{TBA}_{2} \mathrm{H}\left[\left(\alpha_{2}\right) \mathrm{Sn}\left(\mathrm{CH}_{2}\right)_{2} \mathrm{CONHCH}_{2}\left(\mathrm{C}_{2} \mathrm{HN}_{3}\right) \mathrm{Bn}\right]$ \\
2 & $4-$ & 1192.4 & 1192.6 & 20.4 & $\mathrm{TBAH}_{2}\left[\left(\alpha_{2}\right) \mathrm{Sn}\left(\mathrm{CH}_{2}\right)_{2} \mathrm{CONHCH}_{2}\left(\mathrm{C}_{2} \mathrm{HN}_{3}\right) \mathrm{Bn}\right]$ \\
3 & $4-$ & 1132.1 & 1133.0 & 12.1 & $\mathrm{H}_{3}\left[\left(\alpha_{2}\right) \mathrm{Sn}\left(\mathrm{CH}_{2}\right)_{2} \mathrm{CONHCH}_{2}\left(\mathrm{C}_{2} \mathrm{HN}_{3}\right) \mathrm{Bn}\right]$ \\
4 & $5-$ & 1002.1 & 1002.1 & 15.5 & $\mathrm{TBA}_{2}\left[\left(\alpha_{2}\right) \mathrm{Sn}\left(\mathrm{CH}_{2}\right)_{2} \mathrm{CONHCH}_{2}\left(\mathrm{C}_{2} \mathrm{HN}_{3}\right) \mathrm{Bn}\right]$ \\
5 & $5-$ & 953.6 & 953.5 & 100 & $\left.\mathrm{TBAH}_{(}\left(\alpha_{2}\right) \mathrm{Sn}\left(\mathrm{CH}_{2}\right)_{2} \mathrm{CONHCH}_{2}\left(\mathrm{C}_{2} \mathrm{HN}_{3}\right) \mathrm{Bn}\right]$ \\
6 & $5-$ & 905.4 & 905.5 & 48.2 & $\mathrm{H}_{2}\left[\left(\alpha_{2}\right) \operatorname{Sn}\left(\mathrm{CH}_{2}\right)_{2} \mathrm{CONHCH}_{2}\left(\mathrm{C}_{2} \mathrm{HN}_{3}\right) \mathrm{Bn}\right]$ \\
\hline
\end{tabular}




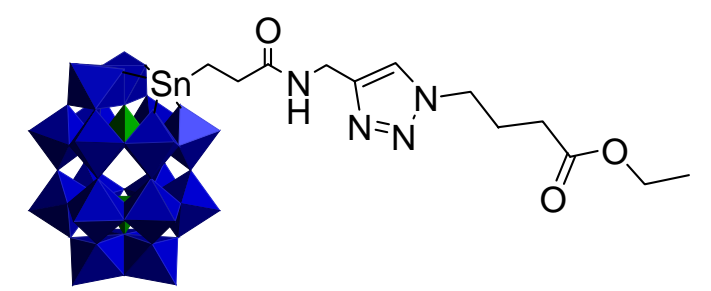

8: $\mathrm{TBA}_{6.4} \mathrm{H}_{0.6}\left[\left(\boldsymbol{\alpha}_{2}\right) \operatorname{Sn}\left(\mathrm{CH}_{2}\right)_{2} \mathrm{CONHCH}_{2}\left(\mathrm{C}_{2} \mathrm{HN}_{3}\right)\left(\mathrm{CH}_{2}\right)_{3} \mathrm{CO}_{2} \mathrm{Et}\right]$. Following GPA, 8 was isolated as a white powder (142 mg, $90 \%)$. IR: $\tilde{\boldsymbol{v}}=2959(\mathrm{~m}), 2934(\mathrm{w}), 2871(\mathrm{~m}), 1649(\mathrm{w}), 1483(\mathrm{~m}), 1378(\mathrm{w})$, 1087 (s), 945 (s), 898 (s), 770 (vs) cm ${ }^{-1} ;{ }^{1} \mathrm{H}$ NMR (400 MHz, $\mathrm{CD}_{3} \mathrm{CN}$ ): $\delta 0.96$ (t, $J=7.1 \mathrm{~Hz}, 79 \mathrm{H}$, $\left.\mathrm{N}\left(\mathrm{CH}_{2} \mathrm{CH}_{2} \mathrm{CH}_{2} \mathrm{Me}\right)_{4}+\mathrm{SnCH}_{2}\right), 1.19\left(\mathrm{t}, J=7.2 \mathrm{~Hz}, 3 \mathrm{H}, M e \mathrm{CH}_{2} \mathrm{O}\right), 1.38-1.44(\mathrm{~m}, 51 \mathrm{H}$, $\left.\mathrm{N}\left(\mathrm{CH}_{2} \mathrm{CH}_{2} \mathrm{CH}_{2} \mathrm{Me}\right)_{4}\right), 1.55-1.70\left(\mathrm{~m}, 51 \mathrm{H}, \mathrm{N}\left(\mathrm{CH}_{2} \mathrm{CH}_{2} \mathrm{CH}_{2} \mathrm{Me}\right)_{4}\right), 2.10-2.15$ (m, $\left.2 \mathrm{H}, \mathrm{CH}_{2} \mathrm{CH}_{2} \mathrm{CH}_{2}\right)$, $2.28\left(\mathrm{t}, J=7.6 \mathrm{~Hz}, 2 \mathrm{H}, \mathrm{CH}_{2} \mathrm{C}(=\mathrm{O}) \mathrm{O}\right), 2.53\left(\mathrm{t}, J=6.8 \mathrm{~Hz}, 2 \mathrm{H}, \mathrm{CH}_{2} \mathrm{C}(=\mathrm{O}) \mathrm{N}\right), 3.08-3.25(\mathrm{~m}, 51 \mathrm{H}$, $\left.\mathrm{N}\left(\mathrm{CH}_{2} \mathrm{Pr}\right)_{4}\right), 4.05\left(\mathrm{q}, J=6.8 \mathrm{~Hz}, 2 \mathrm{H}, \mathrm{OCH}_{2}\right), 4.39\left(\mathrm{~d}, J=5.6 \mathrm{~Hz}, 2 \mathrm{H}, \mathrm{NHCH}_{2}\right), 4.48(\mathrm{t}, J=6.4 \mathrm{~Hz}$, $\left.2 \mathrm{H}, \mathrm{NCH}_{2}\right), 8.08$ (bs, $\left.\left.1 \mathrm{H}, \mathrm{C}=\mathrm{CHN}\right) ;{ }^{13} \mathrm{C} \mathrm{NMR}\left(100 \mathrm{MHz}, \mathrm{CD}_{3} \mathrm{CN}\right): \delta 13.9\left(\mathrm{~N}^{2}\left[\mathrm{CH}_{2}\right]_{3} \mathrm{Me}\right)_{4}\right), 14.4$ $\left.\left.\left(\mathrm{MeCH} \mathrm{CH}_{2} \mathrm{O}\right), 19.3\left(\mathrm{SnCH}_{2}\right), 20.3\left(\mathrm{~N}^{2} \mathrm{CH}_{2}\right]_{2} \mathrm{CH}_{2} \mathrm{Me}\right)_{4}\right), 24.3\left(\mathrm{~N}\left(\mathrm{CH}_{2} \mathrm{CH}_{2} \mathrm{CH}_{2} \mathrm{Me}\right)_{4}\right), 26.4\left(\mathrm{CH}_{2} \mathrm{CH}_{2} \mathrm{CH}_{2}\right)$, $31.5\left(\mathrm{CH}_{2} \mathrm{C}(=\mathrm{O}) \mathrm{O}\right), 32.4\left(\mathrm{CH}_{2} \mathrm{C}(=\mathrm{O}) \mathrm{N}\right), 36.3\left(\mathrm{NHCH}_{2}\right), 49.6\left(\mathrm{NCH}_{2}\right), 59.0\left(\mathrm{~N}\left(\mathrm{CH}_{2} \mathrm{Pr}\right)_{4}\right), 60.9\left(\mathrm{OCH}_{2}\right)$, $124.4(\mathrm{C}=C H \mathrm{~N}), 146.8(C=\mathrm{CHN}), 173.4(\mathrm{C}=\mathrm{O}) \mathrm{O}), 175.1(\mathrm{C}(=\mathrm{O}) \mathrm{NH}) ;{ }^{31} \mathrm{P} \mathrm{NMR}\left(162 \mathrm{MHz}, \mathrm{CD}_{3} \mathrm{CN}\right)$ : $\delta$-9.5 $\left(\mathrm{s}+\mathrm{d}, 1 \mathrm{P}, J_{S n P}=25.9 \mathrm{~Hz}\right),-12.6(\mathrm{bs}, 1 \mathrm{P})$; elemental analysis $(\%)$ for $\mathrm{C}_{114.4} \mathrm{H}_{250} \mathrm{~N}_{10.4} \mathrm{O}_{64} \mathrm{P}_{2} \mathrm{SnW}_{17}$ $\left(6101.77 \mathrm{~g} \cdot \mathrm{mol}^{-1}\right)$ : calc. C 22.52, H 4.13 N 2.39; found C 22.52, H 4.05 N 2.34.

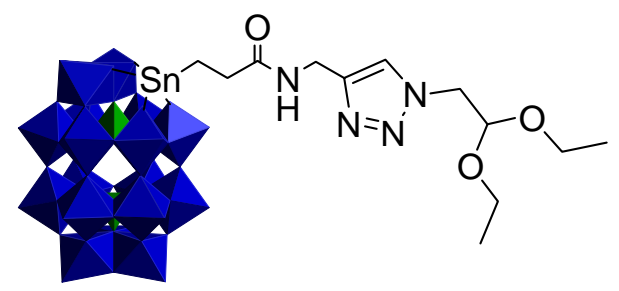

9: $\mathrm{TBA}_{6.2} \mathrm{H}_{0.8}\left[\left(\alpha_{2}\right) \operatorname{Sn}\left(\mathrm{CH}_{2}\right)_{2} \mathrm{CONHCH}_{2}\left(\mathrm{C}_{2} \mathrm{HN}_{3}\right) \mathrm{CH}_{2} \mathrm{CH}(\mathrm{OEt})_{2}\right]$. Following GPA, 9 was isolated as a white powder (146 mg, $93 \%)$. IR: $\tilde{\boldsymbol{v}}=2962(\mathrm{~m}), 2934(\mathrm{w}), 2873(\mathrm{~m}), 1634(\mathrm{w}), 1484(\mathrm{~m}), 1379(\mathrm{w})$, 1090 (s), 948 (s), 902 (s), 777 (vs) cm ${ }^{-1} ;{ }^{1} \mathrm{H}$ NMR (400 MHz, CD ${ }_{3} \mathrm{CN}$ ): $\delta 0.96$ (t, $J=7.1 \mathrm{~Hz}, 75 \mathrm{H}$, $\left.\mathrm{N}\left[\left(\mathrm{CH}_{2}\right)_{3} \mathrm{Me}\right]_{4}\right), \quad 1.09\left(\mathrm{t}, \quad J=7.2 \mathrm{~Hz}, \quad 8 \mathrm{H}, \quad M e \mathrm{CH}_{2} \mathrm{O}+\mathrm{SnCH}_{2}\right), \quad 1.38-1.44 \quad(\mathrm{~m}, \quad 50 \mathrm{H}$, $\left.\mathrm{N}\left(\mathrm{CH}_{2} \mathrm{CH}_{2} \mathrm{CH}_{2} \mathrm{Me}\right)_{4}\right), 1.55-1.70\left(\mathrm{~m}, 50 \mathrm{H}, \mathrm{N}\left(\mathrm{CH}_{2} \mathrm{CH}_{2} \mathrm{CH}_{2} \mathrm{Me}\right)_{4}\right), 2.53\left(\mathrm{t}, J=7.2 \mathrm{~Hz}, 2 \mathrm{H}, \mathrm{CH}_{2} \mathrm{C}=\mathrm{O}\right)$, 3.08-3.25 (m, $\left.50 \mathrm{H}, \mathrm{N}\left(\mathrm{CH}_{2} \mathrm{CH}_{2} \mathrm{CH}_{2} \mathrm{Me}\right)_{4}\right), 3.49\left(\mathrm{~m}, 2 \mathrm{H}, \mathrm{OCH}_{2} \mathrm{Me}\right), 3.72\left(\mathrm{~m}, 2 \mathrm{H}, \mathrm{OCH}_{2} \mathrm{Me}\right), 4.40(\mathrm{~d}$, $\left.J=5.8 \mathrm{~Hz}, 2 \mathrm{H}, \mathrm{NHCH}_{2}\right), 4.43\left(\mathrm{~d}, J=5.6 \mathrm{~Hz}, 2 \mathrm{H}, \mathrm{CH}_{2} \mathrm{CH}\right), 4.94\left(\mathrm{t}, J=5.6 \mathrm{~Hz}, 1 \mathrm{H}, \mathrm{CH}_{2} \mathrm{CH}\right), 7.95$

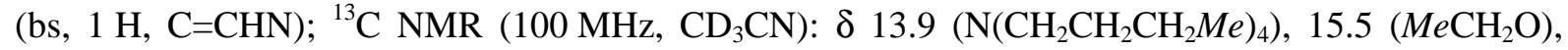

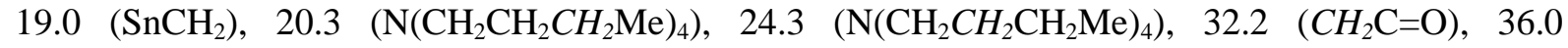
$\left(\mathrm{NHCH}_{2}\right), 52.9\left(\mathrm{NCH}_{2} \mathrm{CH}\right), 59.0\left(\mathrm{~N}\left(\mathrm{CH}_{2} \mathrm{Pr}\right)_{4}\right), 63.6\left(\mathrm{OCH}_{2} \mathrm{Me}\right), 101.2\left(\mathrm{CH}_{2} \mathrm{CH}\right), 124.5(\mathrm{C}=\mathrm{CHN})$, $146.5(C=\mathrm{CHN}), 175.2(\mathrm{C}=\mathrm{O}) ;{ }^{31} \mathrm{P}$ NMR $\left(162 \mathrm{MHz}, \mathrm{CD}_{3} \mathrm{CN}\right): \delta-9.49\left(\mathrm{~s}+\mathrm{d}, 1 \mathrm{P}, J_{S n P}=29.0 \mathrm{~Hz}\right)$, 12.44 (bs, $1 \mathrm{P}$ ); elemental analysis (\%) for $\mathrm{C}_{111.2} \mathrm{H}_{245} \mathrm{~N}_{10.2} \mathrm{O}_{64} \mathrm{P}_{2} \mathrm{SnW}_{17}\left(6055.50 \mathrm{~g} \cdot \mathrm{mol}^{-1}\right)$ : calc. C 22.06, H 4.08, N 2.36; found C 22.16, H 3.99, N 2.26. 


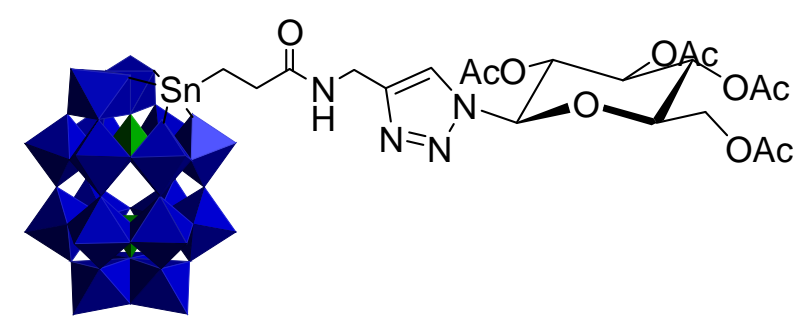

10: $\mathbf{T B A}_{6.5} \mathbf{H}_{0.5}\left[\left(\boldsymbol{\alpha}_{2}\right) \operatorname{Sn}\left(\mathrm{CH}_{2}\right)_{2} \mathrm{CONHCH}_{2}\left(\mathrm{C}_{2} \mathbf{H N}_{3}\right) \mathbf{G l c}(\mathrm{OAc})_{4}\right]$. Following GPA, 10 was isolated as a white powder $(138 \mathrm{mg}, 85 \%)$. IR: $\tilde{\boldsymbol{v}}=2961(\mathrm{~m}), 2932(\mathrm{w}), 2872(\mathrm{~m}), 1753(\mathrm{~m}), 1646(\mathrm{w}), 1508(\mathrm{~m})$, 1378 (w), 1226 (m), 1088 (s), 948 (s), 900 (s), 777 (vs) cm ${ }^{-1} ;{ }^{1} \mathrm{H}$ NMR (400 MHz, $\mathrm{CD}_{3} \mathrm{CN}$ ): $\delta 0.96$ (t, $\left.J=7.1 \mathrm{~Hz}, 84 \mathrm{H}, \mathrm{N}\left(\mathrm{CH}_{2} \mathrm{CH}_{2} \mathrm{CH}_{2} \mathrm{Me}\right)_{4}\right), 1.38-1.44$ (m, $\left.58 \mathrm{H}, \mathrm{N}\left(\mathrm{CH}_{2} \mathrm{CH}_{2} \mathrm{CH}_{2} \mathrm{Me}\right)_{4}+\mathrm{SnCH}_{2}\right), 1.55-1.70$ (m, $\left.56 \mathrm{H}, \mathrm{N}\left(\mathrm{CH}_{2} \mathrm{CH}_{2} \mathrm{CH}_{2} \mathrm{Me} \text { ) }\right)_{4}\right), 1.80$ (s, $3 \mathrm{H}, \mathrm{OAc}$ ), 1.93 (s, $\left.3 \mathrm{H}, \mathrm{OAc}\right), 1.98$ (s, $3 \mathrm{H}, \mathrm{OAc}$ ), 1.99 (s, $3 \mathrm{H}, \mathrm{OAc}), 2.55\left(\mathrm{t}, J=6.4 \mathrm{~Hz}, 2 \mathrm{H}, \mathrm{CH}_{2} \mathrm{C}=\mathrm{O}\right), 3.08-3.25\left(\mathrm{~m}, 56 \mathrm{H}, \mathrm{N}\left(\mathrm{CH}_{2} \mathrm{Pr}\right)_{4}\right), 4.13(\mathrm{~d}, J=12.6 \mathrm{~Hz}$, $1 \mathrm{H}, C H \mathrm{OAc}$ ), 4.20-4.23 (m, $1 \mathrm{H}, C H \mathrm{OAc}$ ), 4.26-4.30 (m, $1 \mathrm{H}, C H \mathrm{OAc}$ ), 4.35 (B of ABX, $J=12.4$, $5.8 \mathrm{~Hz}, 1 \mathrm{H}, \mathrm{CHHOAc}$ ), 4.45 (A of ABX, $J=12.4,5.8 \mathrm{~Hz}, 1 \mathrm{H}, \mathrm{CHHOAc}), 5.20(\mathrm{t}, J=9.6 \mathrm{~Hz}, 1 \mathrm{H}$, $C H \mathrm{OAc}), 5.37(\mathrm{t}, J=9.6 \mathrm{~Hz}, 1 \mathrm{H}, C H \mathrm{OAc}), 5.70(\mathrm{t}, J=9.6 \mathrm{~Hz}, 1 \mathrm{H}, C H \mathrm{OAc}), 6.20(\mathrm{~d}, J=8.4 \mathrm{~Hz}$, $1 \mathrm{H}, \quad \mathrm{NCHO}), 8.21(\mathrm{~s}, 1 \mathrm{H}, \mathrm{C}=\mathrm{CHN}) ;{ }^{13} \mathrm{C} \mathrm{NMR}\left(50 \mathrm{MHz}, \mathrm{CD}_{3} \mathrm{CN}+5 \% \mathrm{D}_{2} \mathrm{O}\right): \delta 13.6$

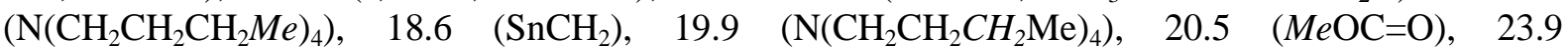
$\left(\mathrm{N}\left(\mathrm{CH}_{2} \mathrm{CH}_{2} \mathrm{CH}_{2} \mathrm{Me}\right)_{4}\right), 31.5\left(\mathrm{CH}_{2} \mathrm{C}=\mathrm{O}\right), 35.4\left(\mathrm{NHCH}_{2}\right), 58.7\left(\mathrm{~N}\left(\mathrm{CH}_{2} \mathrm{CH}_{2} \mathrm{CH}_{2} \mathrm{Me}\right)_{4}\right), 62.0\left(\mathrm{CH}_{2} \mathrm{OAc}\right)$, $68.1(\mathrm{CHO}), 70.5(\mathrm{CHO}), 73.4(\mathrm{CHO}), 74.7(\mathrm{CHO}), 85.0(\mathrm{NCHO}), 124.1(\mathrm{C}=\mathrm{CHN}), 146.6(\mathrm{C}=\mathrm{CHN})$, $169.9(\mathrm{Me} C=\mathrm{O}), 170.5(\mathrm{Me} C=\mathrm{O}), 170.7(\mathrm{Me} C=\mathrm{O}), 171.4(\mathrm{Me} C=\mathrm{O}), 176.0(\mathrm{C}(=\mathrm{O}) \mathrm{NH}) ;{ }^{31} \mathrm{P}$ NMR $\left(162 \mathrm{MHz}, \mathrm{CD}_{3} \mathrm{CN}+5 \% \mathrm{D}_{2} \mathrm{O}\right): \delta-9.4\left(\mathrm{~s}+\mathrm{d}, 1 \mathrm{P}, J_{S n P}=24.3 \mathrm{~Hz}\right),-12.4(\mathrm{~s}, 1 \mathrm{P}) ;[\alpha]_{\mathrm{D}}{ }^{20}-0.7$ (c 2.5, Acetone). ; elemental analysis (\%) for $\mathrm{C}_{124} \mathrm{H}_{261.5} \mathrm{~N}_{10.5} \mathrm{O}_{71} \mathrm{P}_{2} \mathrm{SnW}_{17}\left(6342.07 \mathrm{~g} \cdot \mathrm{mol}^{-1}\right)$ : calc. C 23.48, $\mathrm{H}$ 4.16, N 2.32; found C 23.59, H 4.37, N 2.14.

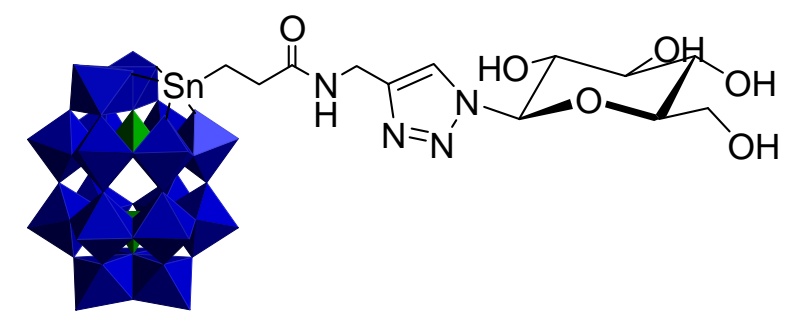

11: $\mathbf{T B A}_{6.5} \mathbf{H}_{0.5}\left[\left(\boldsymbol{\alpha}_{2}\right) \operatorname{Sn}\left(\mathbf{C H}_{2}\right)_{2} \mathbf{C O N H C H}_{2}\left(\mathbf{C}_{2} \mathbf{H N}_{3}\right)\right.$ Glc]. Following GPA with 30 equiv. of sodium ascorbate, 11 was isolated after $48 \mathrm{~h}$ as a white powder $(134 \mathrm{mg}, 85 \%)$. IR: $\tilde{\boldsymbol{v}}=2961(\mathrm{~m}), 2933(\mathrm{w})$, 2872 (m), 1646 (w), 1484 (m), 1379 (w), 1088 (s), 947 (s), 899 (s), 777 (vs) cm ${ }^{-1}$; ${ }^{1} \mathrm{H}$ NMR (400 MHz, $\left.\mathrm{CD}_{3} \mathrm{CN}+5 \% \mathrm{D}_{2} \mathrm{O}\right): \delta 0.96\left(\mathrm{t}, J=7.1 \mathrm{~Hz}, 84 \mathrm{H}, \mathrm{N}\left(\mathrm{CH}_{2} \mathrm{CH}_{2} \mathrm{CH}_{2} \mathrm{Me}\right)_{4}\right), 1.15(\mathrm{t}, J=6.8 \mathrm{~Hz}, 2 \mathrm{H}$, $\left.\mathrm{SnCH}_{2}\right), 1.38-1.44\left(\mathrm{~m}, 56 \mathrm{H}, \mathrm{N}\left(\mathrm{CH}_{2} \mathrm{CH}_{2} \mathrm{CH}_{2} \mathrm{Me}\right)_{4}\right), 1.55-1.70\left(\mathrm{~m}, 56 \mathrm{H}, \mathrm{N}\left(\mathrm{CH}_{2} \mathrm{CH}_{2} \mathrm{CH}_{2} \mathrm{Me}\right)_{4}\right), 2.57$ (t, $\left.J=6.4 \mathrm{~Hz}, 2 \mathrm{H}, \mathrm{CH}_{2} \mathrm{C}=\mathrm{O}\right), 3.08-3.25\left(\mathrm{~m}, 56 \mathrm{H}, \mathrm{N}\left(\mathrm{CH}_{2} \mathrm{Pr}\right)_{4}\right), 3.49-3.77\left(\mathrm{~m}, 5 \mathrm{H}, \mathrm{CHOH}+\mathrm{CHCH}_{2} \mathrm{OH}\right.$ $\left.+\mathrm{CHCH}_{2} \mathrm{OH}\right), 3.89(\mathrm{t}, J=9.2 \mathrm{~Hz}, 1 \mathrm{H}, \mathrm{CHOH}), 4.42\left(\mathrm{AB}, 2 \mathrm{H}, \mathrm{NHCH}_{2}\right), 5.70(\mathrm{t}, J=9.2 \mathrm{~Hz}, 1 \mathrm{H}$, $\mathrm{NCHO}), \quad 8.20 \quad(\mathrm{~s}, \quad 1 \mathrm{H}, \quad \mathrm{C}=\mathrm{CHN}) ; \quad \mathrm{RMN} \quad{ }^{13} \mathrm{C} \quad\left(50 \mathrm{MHz}, \quad \mathrm{CD}_{3} \mathrm{CN}+5 \% \quad \mathrm{D}_{2} \mathrm{O}\right): \delta 13.6$

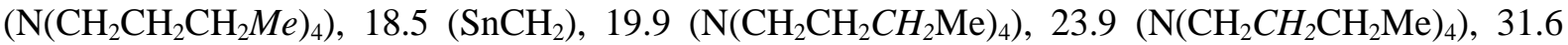
$\left(\mathrm{CH}_{2} \mathrm{C}=\mathrm{O}\right), 35.6\left(\mathrm{NHCH}_{2}\right), 58.7\left(\mathrm{~N}\left(\mathrm{CH}_{2} \mathrm{Pr}\right)_{4}\right), 61.5\left(\mathrm{CH}_{2} \mathrm{O}\right), 70.0(\mathrm{CHO}), 73.1(\mathrm{CHO}), 77.3(\mathrm{CHO})$, $79.7(\mathrm{CHO}), 88.2(\mathrm{NCHO}), 123.4(\mathrm{C}=\mathrm{CHN}), 146.0(\mathrm{C}=\mathrm{CHN}), 176.1 \quad(\mathrm{C}(=\mathrm{O}) \mathrm{NH}) ;{ }^{31} \mathrm{P} \mathrm{NMR}$ $\left(162 \mathrm{MHz}, \mathrm{CD}_{3} \mathrm{CN}+5 \% \mathrm{D}_{2} \mathrm{O}\right): \delta-9.4\left(\mathrm{~s}+\mathrm{d}, 1 \mathrm{P}, J_{S n P}=22.7 \mathrm{~Hz}\right),-12.5(\mathrm{~s}, 1 \mathrm{P})$; elemental analysis $(\%)$ for $\mathrm{C}_{116} \mathrm{H}_{253.5} \mathrm{~N}_{10.5} \mathrm{O}_{67} \mathrm{P}_{2} \mathrm{SnW}_{17}\left(6173.9 \mathrm{~g} \cdot \mathrm{mol}^{-1}\right)$ : calc. C 22.57, H 4.14, N 2.38, P 1.00, W 50.63, Sn 1.92; found C 22.60, H 4.36, N 2.15, P 0.92, W 46.33, Sn 1.92; ESI/MS: see below for full details. 


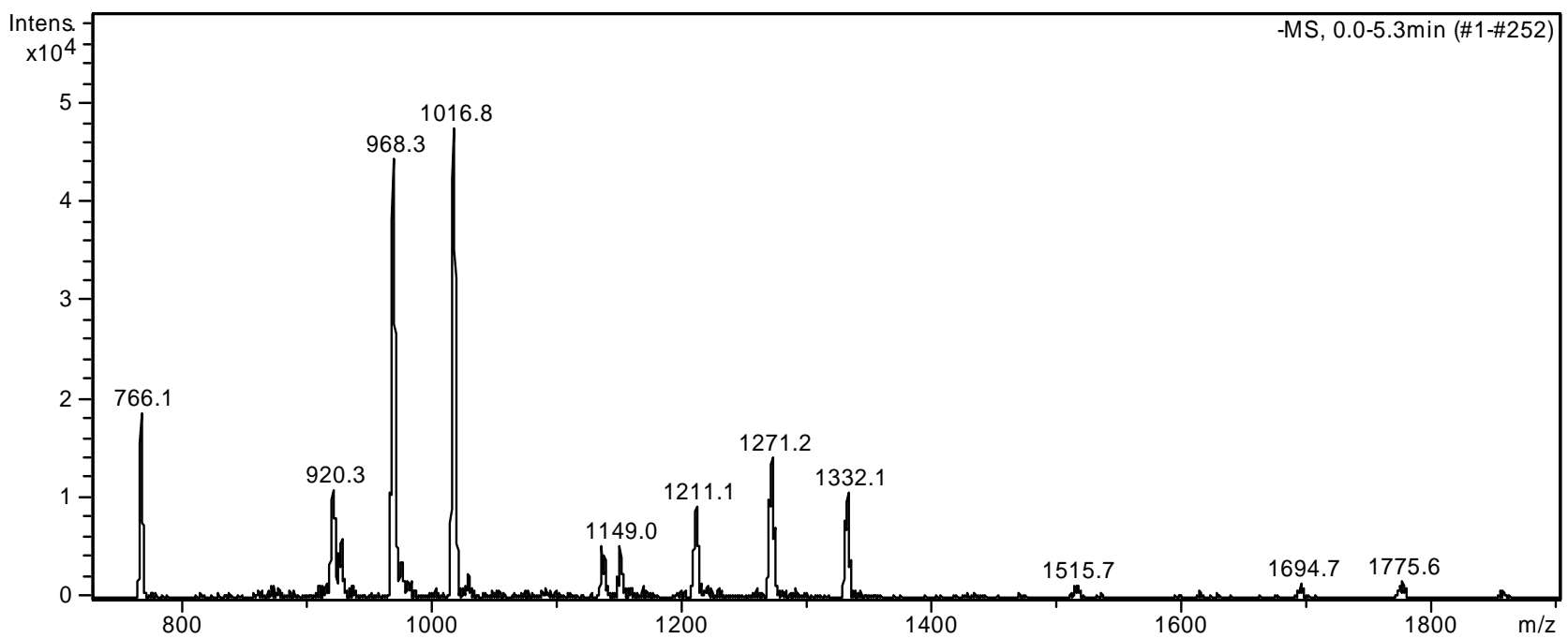

\begin{tabular}{|c|c|c|c|c|c|}
\hline Entry & Charge & $\begin{array}{c}\text { Simulated } \\
\mathrm{m} / \mathrm{z}\end{array}$ & $\begin{array}{c}\text { Observed } \\
\mathrm{m} / \mathrm{z}\end{array}$ & $\begin{array}{l}\text { Relative } \\
\text { intensity }\end{array}$ & Composition \\
\hline 1 & $3-$ & 1855.6 & 1854.7 & 1.9 & $\mathrm{TBA}_{4}\left[\left(\alpha_{2}\right) \operatorname{Sn}\left(\mathrm{CH}_{2}\right)_{2} \mathrm{CONHCH}_{2}\left(\mathrm{C}_{2} \mathrm{HN}_{3}\right) \mathrm{Glc}\right]$ \\
\hline 2 & $3-$ & 1775.3 & 1775.6 & 3.8 & $\mathrm{TBA}_{3} \mathrm{H}\left[\left(\alpha_{2}\right) \mathrm{Sn}\left(\mathrm{CH}_{2}\right)_{2} \mathrm{CONHCH}_{2}\left(\mathrm{C}_{2} \mathrm{HN}_{3}\right) \mathrm{Glc}\right]$ \\
\hline 3 & $3-$ & 1694.8 & 1694.7 & 3.1 & $\mathrm{TBA}_{2} \mathrm{H}_{2}\left[\left(\alpha_{2}\right) \mathrm{Sn}\left(\mathrm{CH}_{2}\right)_{2} \mathrm{CONHCH}_{2}\left(\mathrm{C}_{2} \mathrm{HN}_{3}\right) \mathrm{Glc}\right]$ \\
\hline 4 & 4- & 1331.2 & 1332.1 & 22.4 & $\mathrm{TBA}_{3}\left[\left(\alpha_{2}\right) \mathrm{Sn}\left(\mathrm{CH}_{2}\right)_{2} \mathrm{CONHCH}_{2}\left(\mathrm{C}_{2} \mathrm{HN}_{3}\right) \mathrm{Glc}\right]$ \\
\hline 5 & 4- & 1270.9 & 1271.2 & 30.1 & $\mathrm{TBA}_{2} \mathrm{H}\left[\left(\alpha_{2}\right) \mathrm{Sn}\left(\mathrm{CH}_{2}\right)_{2} \mathrm{CONHCH}_{2}\left(\mathrm{C}_{2} \mathrm{HN}_{3}\right) \mathrm{Glc}\right]$ \\
\hline 6 & 4- & 1210.3 & 1211.1 & 19.5 & $\mathrm{TBAH}_{2}\left[\left(\alpha_{2}\right) \mathrm{Sn}\left(\mathrm{CH}_{2}\right)_{2} \mathrm{CONHCH}_{2}\left(\mathrm{C}_{2} \mathrm{HN}_{3}\right) \mathrm{Glc}\right]$ \\
\hline 7 & $4-$ & 1150.0 & 1149.0 & 10.2 & $\mathrm{H}_{3}\left[\left(\alpha_{2}\right) \mathrm{Sn}\left(\mathrm{CH}_{2}\right)_{2} \mathrm{CONHCH}_{2}\left(\mathrm{C}_{2} \mathrm{HN}_{3}\right) \mathrm{Glc}\right]$ \\
\hline 8 & $5-$ & 1016.5 & 1016.8 & 100 & $\mathrm{TBA}_{2}\left[\left(\alpha_{2}\right) \mathrm{Sn}\left(\mathrm{CH}_{2}\right)_{2} \mathrm{CONHCH}_{2}\left(\mathrm{C}_{2} \mathrm{HN}_{3}\right) \mathrm{Glc}\right]$ \\
\hline 9 & $5-$ & 968.1 & 968.3 & 93.5 & $\operatorname{TBAH}\left[\left(\alpha_{2}\right) \operatorname{Sn}\left(\mathrm{CH}_{2}\right)_{2} \mathrm{CONHCH}_{2}\left(\mathrm{C}_{2} \mathrm{HN}_{3}\right) \mathrm{Glc}\right]$ \\
\hline 10 & $5-$ & 919.9 & 920.3 & 23.4 & $\mathrm{H}_{2}\left[\left(\alpha_{2}\right) \operatorname{Sn}\left(\mathrm{CH}_{2}\right)_{2} \mathrm{CONHCH}_{2}\left(\mathrm{C}_{2} \mathrm{HN}_{3}\right) \mathrm{Glc}\right]$ \\
\hline 11 & $6-$ & 766.3 & 766.1 & 39.7 & $\mathrm{H}\left[\left(\alpha_{2}\right) \mathrm{Sn}\left(\mathrm{CH}_{2}\right)_{2} \mathrm{CONHCH}_{2}\left(\mathrm{C}_{2} \mathrm{HN}_{3}\right) \mathrm{Glc}\right]$ \\
\hline
\end{tabular}

$\mathbf{K}_{6.5} \mathbf{T M A}_{0.5}\left[\left(\boldsymbol{\alpha}_{2}\right) \operatorname{Sn}\left(\mathrm{CH}_{2}\right)_{2} \mathrm{CONHCH}_{2}\left(\mathbf{C}_{2} \mathbf{H N}_{3}\right) \mathbf{G l c}\right]$. To a solution of $11(0.022 \mathrm{mmol}, 134 \mathrm{mg})$ in $\mathrm{CH}_{3} \mathrm{CN}(2 \mathrm{~mL})$ was added a saturated solution of $\mathrm{TMABr}$ in $\mathrm{CH}_{3} \mathrm{CN}(5-10 \mathrm{~mL})$. A white precipitate appeared, which was isolated by centrifugation. The solid was dissolved in water and treated with cation-exchange resin (Amberlyst, 16-50 mesh, $\mathrm{K}^{+}$form). Filtration and removal of water in vacuo afforded K-11 as a white powder (72 mg, $67 \%) .{ }^{1} \mathrm{H}$ NMR $\left(400 \mathrm{MHz}, \mathrm{D}_{2} \mathrm{O}\right): \delta 1.61(\mathrm{t}, J=7.3 \mathrm{~Hz}, 2 \mathrm{H}$, $\mathrm{SnCH}_{2}$ ), 2.87 (t, $J=7.1 \mathrm{~Hz}, 2 \mathrm{H}, \mathrm{CH}_{2} \mathrm{C}=\mathrm{O}$ ), 3.31 (s, $6 \mathrm{H}, \mathrm{NMe}_{4}$ ), 3.77 (quint., $J=9.4 \mathrm{~Hz}, 2 \mathrm{H}, \mathrm{CH}_{2} \mathrm{O}$ ), 3.85-3.94 (m, $2 \mathrm{H}, 2 \times \mathrm{CHOH}), 4.03(\mathrm{~d}, J=11.9 \mathrm{~Hz}, 1 \mathrm{H}, \mathrm{CHOH}), 4.10(\mathrm{t}, J=8.1 \mathrm{~Hz}, 1 \mathrm{H}, C H \mathrm{OH})$, $4.64\left(\mathrm{~s}, 2 \mathrm{H}, \mathrm{NCH}_{2}\right), 5.90(\mathrm{~d}, J=9.1 \mathrm{~Hz}, \mathrm{NCH}), 8.33(\mathrm{~s}, 1 \mathrm{H}, \mathrm{C}=\mathrm{CHN}) ;{ }^{31} \mathrm{P} \mathrm{NMR}\left(162 \mathrm{MHz}, \mathrm{D}_{2} \mathrm{O}\right): \delta$ $8.5\left(\mathrm{~s}+\mathrm{d}, 1 \mathrm{P}, J_{S n P}=9.3 \mathrm{~Hz}\right),-12.4(\mathrm{~s}, 1 \mathrm{P}) .[\alpha]_{\mathrm{D}}{ }^{20}+2.2\left(\mathrm{c} 0.85, \mathrm{H}_{2} \mathrm{O}\right)$. 


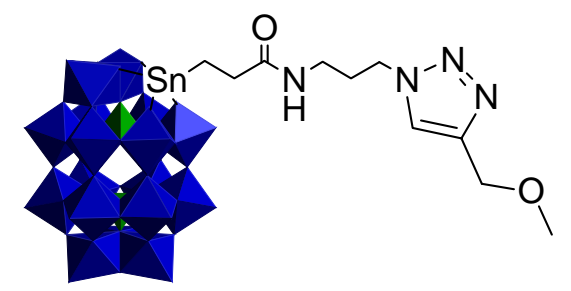

12: $\mathrm{TBA}_{6.3} \mathrm{H}_{0.7}\left[\left(\boldsymbol{\alpha}_{2}\right) \operatorname{Sn}\left(\mathrm{CH}_{2}\right)_{2} \mathrm{CONH}\left(\mathrm{CH}_{2}\right)_{3}\left(\mathrm{~N}_{3} \mathrm{C}_{2} \mathrm{H}\right) \mathrm{CH}_{2} \mathrm{OMe}\right]$. Following GPA, 12 was isolated as a white powder (126 mg, $81 \%)$. IR: $\tilde{\boldsymbol{v}}=2962(\mathrm{~m}), 2934(\mathrm{w}), 2873(\mathrm{~m}), 1653(\mathrm{w}), 1484(\mathrm{~m}), 1379(\mathrm{w})$, 10890 (s), 950 (s), 902 (s), 781 (vs) $\mathrm{cm}^{-1}$; ${ }^{1} \mathrm{H} \mathrm{NMR}$ (400 MHz, $\mathrm{CD}_{3} \mathrm{CN}+5 \% \mathrm{D}_{2} \mathrm{O}$ ): $\delta 0.96$ (t, $\left.J=7.1 \mathrm{~Hz}, \quad 76 \mathrm{H}, \quad \mathrm{N}\left(\mathrm{CH}_{2} \mathrm{CH}_{2} \mathrm{CH}_{2} \mathrm{Me}\right)_{4}\right), \quad 1.05-1.12 \quad\left(\mathrm{~m}, \quad 2 \mathrm{H}, \quad \mathrm{SnCH}_{2}\right), \quad 1.38-1.44 \quad(\mathrm{~m}, \quad 50 \mathrm{H}$, $\left.\mathrm{N}\left(\mathrm{CH}_{2} \mathrm{CH}_{2} \mathrm{CH}_{2} \mathrm{Me}\right)_{4}\right), 1.55-1.70\left(\mathrm{~m}, 50 \mathrm{H}, \mathrm{N}\left(\mathrm{CH}_{2} \mathrm{CH}_{2} \mathrm{CH}_{2} \mathrm{Me}\right)_{4}\right), 2.17-2.20\left(\mathrm{~m}, 2 \mathrm{H}, \mathrm{CH}_{2} \mathrm{CH}_{2} \mathrm{CH}_{2}\right)$, $2.51\left(\mathrm{t}, J=7.2 \mathrm{~Hz}, 2 \mathrm{H}, \mathrm{CH}_{2} \mathrm{C}=\mathrm{O}\right), 3.08-3.25\left(\mathrm{~m}, 52 \mathrm{H}, \mathrm{N}\left(\mathrm{CH}_{2} \mathrm{CH}_{2} \mathrm{CH}_{2} \mathrm{Me}\right)_{4}+\mathrm{NHCH}_{2} \mathrm{CH}_{2}\right), 3.30(\mathrm{~s}$, $3 \mathrm{H}, \mathrm{OMe}), 4.46\left(\mathrm{~s}, 2 \mathrm{H}, \mathrm{CH}_{2} \mathrm{O}\right), 4.50\left(\mathrm{t}, J=6 \mathrm{~Hz}, 2 \mathrm{H}, \mathrm{CH}_{2} \mathrm{~N}\right), 8.03(\mathrm{~s}, 1 \mathrm{H}, \mathrm{C}=\mathrm{CHN}) ;{ }^{13} \mathrm{C} \mathrm{NMR}$

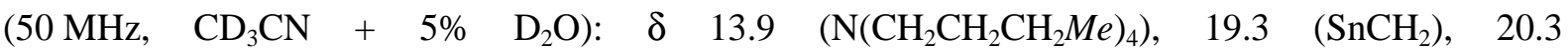
$\left(\mathrm{N}\left(\mathrm{CH}_{2} \mathrm{CH}_{2} \mathrm{CH}_{2} \mathrm{Me}\right)_{4}\right), 24.3 \quad\left(\mathrm{~N}\left(\mathrm{CH}_{2} \mathrm{CH}_{2} \mathrm{CH}_{2} \mathrm{Me}\right)_{4}\right), \quad 30.4 \quad\left(\mathrm{CH}_{2} \mathrm{CH}_{2} \mathrm{CH}_{2}\right), 32.5 \quad\left(\mathrm{CH}_{2} \mathrm{C}=\mathrm{O}\right), \quad 36.9$ $\left(\mathrm{NHCH}_{2}\right), 48.5\left(\mathrm{CH}_{2} \mathrm{CH}_{2} \mathrm{~N}\right), 57.9(\mathrm{MeO}), 59.0\left(\mathrm{~N}\left(\mathrm{CH}_{2} \mathrm{CH}_{2} \mathrm{CH}_{2} \mathrm{Me}\right)_{4}\right), 66.0\left(\mathrm{CH}_{2} \mathrm{O}\right), 125.4(\mathrm{C}=\mathrm{CHN})$, $144.6(C=\mathrm{CHN}), 175.3(\mathrm{C}=\mathrm{O}) ;{ }^{31} \mathrm{P}$ NMR $\left(162 \mathrm{MHz}, \mathrm{CD}_{3} \mathrm{CN}+5 \% \mathrm{D}_{2} \mathrm{O}\right): \delta-9.43(\mathrm{~s}+\mathrm{d}, 1 \mathrm{P}$, $\left.J_{S n P}=24.3 \mathrm{~Hz}\right),-12.50(\mathrm{~s}, 1 \mathrm{P})$; elemental analysis $(\%)$ for $\mathrm{C}_{110.8} \mathrm{H}_{244.5} \mathrm{~N}_{10.3} \mathrm{O}_{63} \mathrm{P}_{2} \mathrm{SnW}_{17}\left(6035.59 \mathrm{~g} \cdot \mathrm{mol}^{-}\right.$ $\left.{ }^{1}\right)$ : calc. C 22.05, H 4.08 N 2.39; found C 22.08, H 3.95, N 2.22.

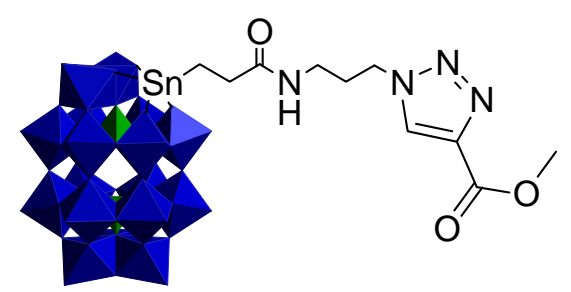

13: $\mathrm{TBA}_{6.6} \mathrm{H}_{0.4}\left[\left(\alpha_{2}\right) \operatorname{Sn}\left(\mathrm{CH}_{2}\right)_{2} \mathbf{C O N H}\left(\mathrm{CH}_{2}\right)_{3}\left(\mathbf{N}_{3} \mathrm{C}_{2} \mathrm{H}\right) \mathrm{CO}_{2} \mathrm{Me}\right]$. Following GPA, 13 was isolated as a white powder (135 mg, $87 \%)$. IR: $\tilde{v}=2960(\mathrm{~m}), 2935(\mathrm{w}), 2871(\mathrm{~m}), 1727(\mathrm{w}), 1648(\mathrm{w}), 1483(\mathrm{~m})$, 1378 (w), 1088 (s), 946 (s), 899 (s), 775 (vs) cm ${ }^{-1}$; ${ }^{1} \mathrm{H}$ NMR (400 MHz, $\mathrm{CD}_{3} \mathrm{CN}+5 \% \mathrm{D}_{2} \mathrm{O}$ ): $\delta 0.96$ (t, $\left.J=7.1 \mathrm{~Hz}, 79 \mathrm{H}, \mathrm{N}\left(\mathrm{CH}_{2} \mathrm{CH}_{2} \mathrm{CH}_{2} \mathrm{Me}\right)_{4}\right), 1.17\left(\mathrm{t}, J=7.6 \mathrm{~Hz}, 2 \mathrm{H}, \quad \mathrm{SnCH}_{2}\right), 1.38-1.44(\mathrm{~m}, 53 \mathrm{H}$, $\left.\mathrm{N}\left(\mathrm{CH}_{2} \mathrm{CH}_{2} \mathrm{CH}_{2} \mathrm{Me}\right)_{4}\right), 1.55-1.70\left(\mathrm{~m}, 53 \mathrm{H}, \mathrm{N}\left(\mathrm{CH}_{2} \mathrm{CH}_{2} \mathrm{CH}_{2} \mathrm{Me}\right)_{4}\right), 2.19\left(\mathrm{~m}, 2 \mathrm{H}, \mathrm{CH}_{2} \mathrm{CH}_{2} \mathrm{CH}_{2}\right), 2.53$ (t, $\left.J=7.6 \mathrm{~Hz}, 2 \mathrm{H}, \mathrm{CH}_{2} \mathrm{C}=\mathrm{O}\right), 3.08-3.25\left(\mathrm{~m}, 55 \mathrm{H}, \mathrm{N}\left(\mathrm{CH}_{2} \mathrm{CH}_{2} \mathrm{CH}_{2} \mathrm{Me}\right)_{4}+\mathrm{NHCH}_{2}\right), 3.85$ (s, $\left.3 \mathrm{H}, \mathrm{OMe}\right)$, $4.55\left(\mathrm{t}, J=6.8 \mathrm{~Hz}, 2 \mathrm{H}, \mathrm{CH}_{2} \mathrm{~N}\right), 8.53(\mathrm{~s}, 1 \mathrm{H}, \mathrm{C}=\mathrm{CHN}) ;{ }^{13} \mathrm{C} \mathrm{NMR}\left(50 \mathrm{MHz}, \mathrm{CD}_{3} \mathrm{CN}+5 \% \mathrm{D}_{2} \mathrm{O}\right): \delta$

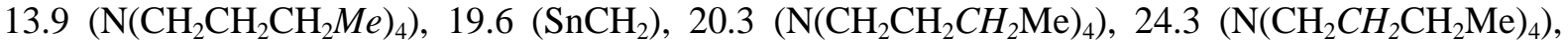
$30.1 \quad\left(\mathrm{CH}_{2} \mathrm{CH}_{2} \mathrm{CH}_{2}\right), 32.7\left(\mathrm{CH}_{2} \mathrm{C}=\mathrm{O}\right), 36.3\left(\mathrm{NHCH}_{2}\right), 48.9 \quad\left(\mathrm{CH}_{2} \mathrm{CH}_{2} \mathrm{~N}\right), 52.2(\mathrm{MeO}), 59.0$ $\left(\mathrm{N}\left(\mathrm{CH}_{2} \mathrm{CH}_{2} \mathrm{CH}_{2} \mathrm{Me}\right)_{4}\right), 130.6(\mathrm{C}=\mathrm{CHN}), 139.4(\mathrm{C}=\mathrm{CHN}), 162.2(\mathrm{C}(=\mathrm{O}) \mathrm{O}), 175.2(\mathrm{C}(=\mathrm{O}) \mathrm{NH}) ;{ }^{31} \mathrm{P}$ NMR $\left(162 \mathrm{MHz}, \mathrm{CD}_{3} \mathrm{CN}+5 \% \mathrm{D}_{2} \mathrm{O}\right): \delta-9.36\left(\mathrm{~s}+\mathrm{d}, 1 \mathrm{P}, J_{S n P}=25.9 \mathrm{~Hz}\right),-12.52(\mathrm{~s}, 1 \mathrm{P})$; elemental analysis (\%) for $\mathrm{C}_{115.6} \mathrm{H}_{253} \mathrm{~N}_{10.6} \mathrm{O}_{64} \mathrm{P}_{2} \mathrm{SnW}_{17}\left(6122.01 \mathrm{~g} \cdot \mathrm{mol}^{-1}\right)$ : calc. $\mathrm{C} 22.68, \mathrm{H} 4.17, \mathrm{~N} 2.43$; found C 22.73, H 4.01, N 2.31. 


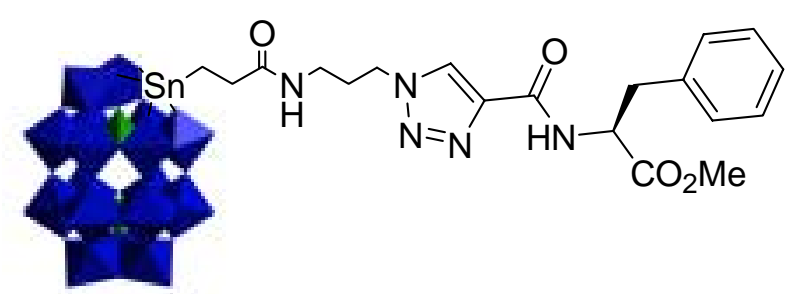

14: $\mathrm{TBA}_{6.7} \mathrm{H}_{0.3}\left[\left(\alpha_{2}\right) \operatorname{Sn}\left(\mathrm{CH}_{2}\right)_{2} \mathbf{C O N H}\left(\mathrm{CH}_{2}\right)_{3}\left(\mathrm{~N}_{3} \mathrm{C}_{2} \mathrm{H}\right) \mathrm{CO}\{\mathrm{Phe}\} \mathrm{OMe}\right]$. Following GPA, 14 was isolated as a white powder (122 mg, $77 \%)$. IR: $\tilde{\boldsymbol{v}}=2961(\mathrm{~m}), 2935(\mathrm{w}), 2873(\mathrm{~m}), 1736(\mathrm{w}), 1658(\mathrm{w}), 1484$ (m), 1379 (w), 1088 (s), 946 (s), 899 (s), 777 (vs) cm ${ }^{-1} ;{ }^{1} \mathrm{H}$ NMR (400 MHz, $\left.\mathrm{CD}_{3} \mathrm{CN}+5 \% \mathrm{D}_{2} \mathrm{O}\right): \delta$ $0.95\left(\mathrm{t}, J=7.1 \mathrm{~Hz}, 84 \mathrm{H}, \mathrm{N}\left(\mathrm{CH}_{2} \mathrm{CH}_{2} \mathrm{CH}_{2} M e\right)_{4}\right), 1.17\left(\mathrm{t}, J=7.6 \mathrm{~Hz}, 2 \mathrm{H}, \mathrm{SnCH}_{2}\right), 1.38-1.44(\mathrm{~m}, 56 \mathrm{H}$, $\left.\mathrm{N}\left(\mathrm{CH}_{2} \mathrm{CH}_{2} \mathrm{CH}_{2} \mathrm{Me}\right)_{4}\right), 1.55-1.70\left(\mathrm{~m}, 56 \mathrm{H}, \mathrm{N}\left(\mathrm{CH}_{2} \mathrm{CH}_{2} \mathrm{CH}_{2} \mathrm{Me}\right)_{4}\right), 2.17-2.20\left(\mathrm{~m}, 2 \mathrm{H}, \mathrm{CH}_{2} \mathrm{CH}_{2} \mathrm{CH}_{2}\right)$, $2.53\left(\mathrm{t}, J=7.6 \mathrm{~Hz}, 2 \mathrm{H}, \mathrm{CH}_{2} \mathrm{C}=\mathrm{O}\right), 3.08-3.25\left(\mathrm{~m}, 60 \mathrm{H}, \mathrm{N}\left(\mathrm{CH}_{2} \mathrm{CH}_{2} \mathrm{CH}_{2} \mathrm{Me}\right)_{4}+\mathrm{NHCH}_{2} \mathrm{CH}_{2}+\mathrm{CH}_{2} \mathrm{Ph}\right)$, $3.67(\mathrm{~s}, 3 \mathrm{H}, \mathrm{OMe}), 4.55\left(\mathrm{t}, J=6.8 \mathrm{~Hz}, 2 \mathrm{H}, \mathrm{CH}_{2} \mathrm{CH}_{2} \mathrm{~N}\right), 4.81-4.84(\mathrm{~m}, 1 \mathrm{H}, \mathrm{NHCHCO}), 7.20-7.29$ (m, $5 \mathrm{H}$ arom.), $7.78(\mathrm{~d}, J=8.1 \mathrm{~Hz}, 1 \mathrm{H}, \mathrm{NH}), 8.42(\mathrm{~s}, 1 \mathrm{H}, \mathrm{C}=\mathrm{CHN}) ;{ }^{13} \mathrm{C} \mathrm{NMR}\left(50 \mathrm{MHz}, \mathrm{CD}_{3} \mathrm{CN}+5 \%\right.$

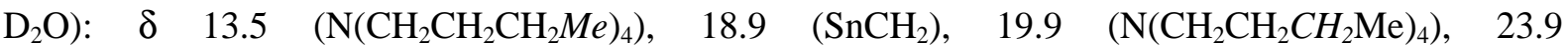
$\left(\mathrm{N}\left(\mathrm{CH}_{2} \mathrm{CH}_{2} \mathrm{CH}_{2} \mathrm{Me}\right)_{4}\right), 29.8\left(\mathrm{CH}_{2} \mathrm{CH}_{2} \mathrm{CH}_{2}\right), 32.0\left(\mathrm{CH}_{2} \mathrm{C}=\mathrm{O}\right), 36.1\left(\mathrm{NHCH}_{2}\right), 37.4\left(\mathrm{CH}_{2} \mathrm{Ph}\right), 48.6$ $\left(\mathrm{CH}_{2} \mathrm{CH}_{2} \mathrm{~N}\right), 52.6(\mathrm{MeO}$ or $\mathrm{NHCHC}=\mathrm{O}), 53.9(\mathrm{MeO}$ or $\mathrm{NHCHC}=\mathrm{O}), 58.6\left(\mathrm{~N}\left(\mathrm{CH}_{2} \mathrm{CH}_{2} \mathrm{CH}_{2} \mathrm{Me}\right)_{4}\right)$, $127.4(\mathrm{CH}$ arom. $), 127.7(\mathrm{C}=\mathrm{CHN}), 129.1(\mathrm{CH}$ arom. $), 129.8(\mathrm{C}$ arom. $), 137.3(\mathrm{CH}$ arom. $), 141.8$ $(C=\mathrm{CHN}), 160.9(\mathrm{C}(=\mathrm{O}) \mathrm{O}), 172.5(\mathrm{C}(=\mathrm{O}) \mathrm{NH}), 176.2(\mathrm{C}(=\mathrm{O}) \mathrm{NH}) ;{ }^{31} \mathrm{P} \mathrm{NMR}\left(162 \mathrm{MHz}, \mathrm{CD}_{3} \mathrm{CN}+5 \%\right.$ $\left.\mathrm{D}_{2} \mathrm{O}\right): \delta-9.4\left(\mathrm{~s}+\mathrm{d}, 1 \mathrm{P}, J_{S n P}=24.3 \mathrm{~Hz}\right),-12.5(\mathrm{~s}, 1 \mathrm{P}) ;[\alpha]_{\mathrm{D}}^{20}-0.8$ (c 2.5, Acetone); elemental analysis (\%) for $\mathrm{C}_{126.2} \mathrm{H}_{265.5} \mathrm{~N}_{11.7} \mathrm{O}_{65} \mathrm{P}_{2} \mathrm{SnW}_{17}\left(6293.33 \mathrm{~g} \cdot \mathrm{mol}^{-1}\right)$ : calc. C 24.09, H 4.25, N 2.60; found C 24.08, $\mathrm{H} 4.38, \mathrm{~N} 2.55$.

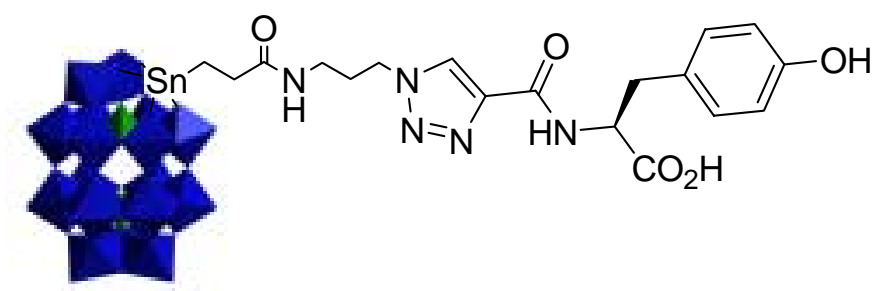

15: $\mathrm{TBA}_{6.9} \mathrm{H}_{0.1}\left[\left(\alpha_{2}\right) \operatorname{Sn}\left(\mathrm{CH}_{2}\right)_{2} \mathrm{CONH}\left(\mathrm{CH}_{2}\right)_{3}\left(\mathrm{~N}_{3} \mathrm{C}_{2} \mathrm{H}\right) \mathrm{CO}\{\mathrm{Tyr}\} \mathrm{OH}\right]$. Following GPA, 15 was isolated as a white powder $(111 \mathrm{mg}, 70 \%)$. IR: $\tilde{\boldsymbol{v}}=2961(\mathrm{~m}), 2935(\mathrm{w}), 2873(\mathrm{~m}), 1654(\mathrm{w}), 1484(\mathrm{~m}), 1379$ (w), 1089 (s), 950 (s), 901 (s), 778 (vs) cm ${ }^{-1}$; ${ }^{1} \mathrm{H}$ NMR (400 MHz, $\mathrm{CD}_{3} \mathrm{CN}+5 \% \mathrm{D}_{2} \mathrm{O}$ ): $\delta 0.95$ (t, $\left.J=7.1 \mathrm{~Hz}, 84 \mathrm{H}, \mathrm{N}\left(\mathrm{CH}_{2} \mathrm{CH}_{2} \mathrm{CH}_{2} \mathrm{Me}\right)_{4}\right), 1.12\left(\mathrm{t}, J=7.6 \mathrm{~Hz}, 2 \mathrm{H}, \mathrm{SnCH}_{2}\right), 1.38-1.44(\mathrm{~m}, 56 \mathrm{H}$, $\left.\mathrm{N}\left(\mathrm{CH}_{2} \mathrm{CH}_{2} \mathrm{CH}_{2} \mathrm{Me}\right)_{4}\right), 1.55-1.70\left(\mathrm{~m}, 56 \mathrm{H}, \mathrm{N}\left(\mathrm{CH}_{2} \mathrm{CH}_{2} \mathrm{CH}_{2} \mathrm{Me}\right)_{4}\right), 2.17-2.28\left(\mathrm{~m}, 2 \mathrm{H}, \mathrm{CH}_{2} \mathrm{CH}_{2} \mathrm{CH}_{2}\right)$, $2.51\left(\mathrm{t}, J=7.4 \mathrm{~Hz}, 2 \mathrm{H}, \mathrm{CH}_{2} \mathrm{C}=\mathrm{O}\right.$ ), $3.00\left(\mathrm{~B}\right.$ of $\left.\mathrm{ABX}, J=14.2,7.8 \mathrm{~Hz}, 1 \mathrm{H}, \mathrm{CHCH}_{2}\right), 3.08-3.25(\mathrm{~m}$, $59 \mathrm{H}, \mathrm{N}\left(\mathrm{CH}_{2} \mathrm{CH}_{2} \mathrm{CH}_{2} \mathrm{Me}\right)_{4}+\mathrm{NHCH}_{2} \mathrm{CH}_{2}+\mathrm{A}$ of $\left.\mathrm{ABX}, \mathrm{CHCH}_{2}\right), 4.59\left(\mathrm{t}, \mathrm{J}=6.3 \mathrm{~Hz}, 2 \mathrm{H}, \mathrm{CH}_{2} \mathrm{CH}_{2} \mathrm{~N}\right)$, $4.66(\mathrm{dd}, J=7.6,5.0 \mathrm{~Hz}, 1 \mathrm{H}, \mathrm{NHCHCO}), 6.72(\mathrm{~d}, J=8.4 \mathrm{~Hz}, 2 \mathrm{H}$, arom.), $7.06(\mathrm{~d}, J=8.6 \mathrm{~Hz}, 2 \mathrm{H}$,

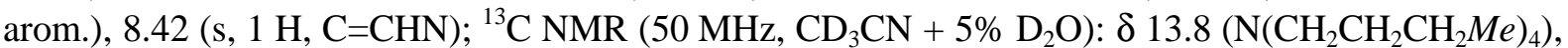

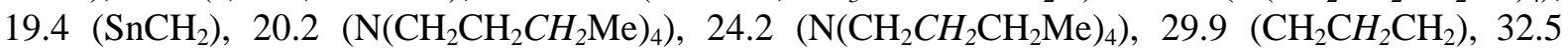

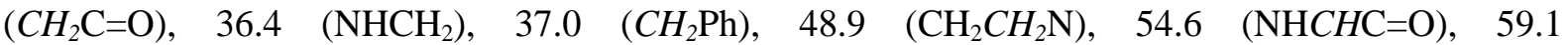
$\left(\mathrm{N}\left(\mathrm{CH}_{2} \mathrm{CH}_{2} \mathrm{CH}_{2} \mathrm{Me}\right)_{4}\right), 116.0(\mathrm{CH}$ arom. $), 127.8(\mathrm{C}=\mathrm{CHN}), 128.6(\mathrm{C}$ arom. $), 131.3(\mathrm{CH}$ arom. $), 142.4$ $(C=\mathrm{CHN}), 156.6(\mathrm{COH}$ arom. $), 161.0(\mathrm{C}=\mathrm{O}) \mathrm{O}), 173.6(\mathrm{C}(=\mathrm{O}) \mathrm{NH}), 176.1(\mathrm{C}(=\mathrm{O}) \mathrm{NH}) ;{ }^{31} \mathrm{P}$ NMR $\left(162 \mathrm{MHz}, \mathrm{CD}_{3} \mathrm{CN}+5 \% \mathrm{D}_{2} \mathrm{O}\right): \delta-9.4\left(\mathrm{~s}+\mathrm{d}, 1 \mathrm{P}, J_{S n P}=26.0 \mathrm{~Hz}\right),-12.7(\mathrm{~s}, 1 \mathrm{P}) ;[\alpha]_{\mathrm{D}}{ }^{20}+3.1(\mathrm{c} 4.75$, Acetone); elemental analysis (\%) for $\mathrm{C}_{128.4} \mathrm{H}_{270.5} \mathrm{~N}_{11.9} \mathrm{O}_{66} \mathrm{P}_{2} \mathrm{SnW}_{17}\left(6343.6 \mathrm{~g} \cdot \mathrm{mol}^{-1}\right)$ : calc. C 24.31, $\mathrm{H}$ 4.30, N 2.63; found C 24.35, H 4.21, N 2.51. 


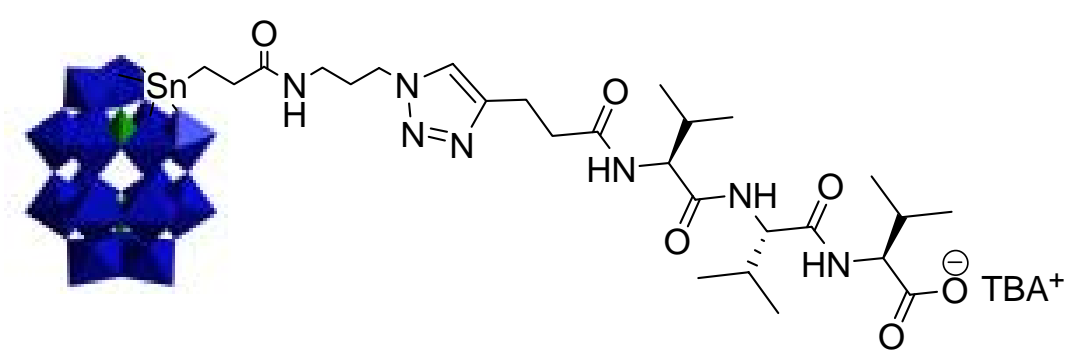

16: $\mathrm{TBA}_{7} \mathrm{H}\left[\left(\boldsymbol{\alpha}_{2}\right) \operatorname{Sn}\left(\mathrm{CH}_{2}\right)_{2} \mathrm{CONH}\left(\mathrm{CH}_{2}\right)_{3}\left(\mathrm{~N}_{3} \mathrm{C}_{2} \mathrm{H}\right)\left(\mathrm{CH}_{2}\right)_{2} \mathrm{CO}\{\right.$ ValValVal $\left.\} \mathrm{O}^{-}\right]$. To a solution of $\mathrm{TBA}_{5}\left[\mathrm{Et}_{3} \mathrm{NH}^{+}\right]_{2}\left[\mathrm{P}_{2} \mathrm{~W}_{17} \mathrm{O}_{61}\left\{\mathrm{SnCH}_{2} \mathrm{CH}_{2} \mathrm{C}(=\mathrm{O}) \mathrm{NH}\left(\mathrm{CH}_{2}\right)_{3} \mathrm{~N}_{3}\right\}\right](\mathbf{2}, 0.035 \mathrm{mmol}, 206 \mathrm{mg})$ in acetonitrile $(1 \mathrm{~mL})$ was added the alkyne $\left(0.070 \mathrm{mmol}, 2\right.$ equiv). A solution of $\mathrm{CuSO}_{4} \cdot 5 \mathrm{H}_{2} \mathrm{O}(0.026 \mathrm{mmol}, 4$ equiv, $35 \mathrm{mg})$ in water $(0.5 \mathrm{~mL})$ and a solution of sodium ascorbate $(0.26 \mathrm{mmol}, 40$ equiv, $277 \mathrm{mg})$ in water $(1.5 \mathrm{~mL})$ were then added. The mixture was stirred at room temp for 4 days. A cation-exchange resin (Amberlyst 15, 16-50 mesh, $\mathrm{TBA}^{+}$form) was added, followed by acetone $(10 \mathrm{~mL})$ and the mixture was stirred for 1 hour or until disappearance of the precipitate (if any appeared). The resin was filtered off and the filtrate was concentrated in vacuo. Dichloromethane $(30 \mathrm{~mL})$, and water $(30 \mathrm{~mL})$ were added to the crude residue. The organic phase was washed three times with water $(30 \mathrm{~mL})$ and concentrated in vacuo to deliver a white solid. This solid was dissolved in acetone $(2 \mathrm{~mL})$, and precipitated upon addition of $\mathrm{EtOH} / \mathrm{Et}_{2} \mathrm{O}(2 \mathrm{~mL} / 30 \mathrm{~mL})$. The solid was isolated by centrifugation, washed with $\mathrm{Et}_{2} \mathrm{O}$ and dried in vacuo to afford the desired POM 16 (155 mg, $68 \%$ ). IR: $\tilde{\boldsymbol{v}}=2961(\mathrm{~m}), 2935(\mathrm{w}), 2873(\mathrm{~m}), 1654(\mathrm{w}), 1484(\mathrm{~m}), 1379(\mathrm{w}), 1089(\mathrm{~s}), 950(\mathrm{~s}), 901(\mathrm{~s}), 778$ (vs) $\mathrm{cm}^{-1} ;{ }^{1} \mathrm{H}$ NMR $\left(400 \mathrm{MHz}, \mathrm{CD}_{3} \mathrm{CN}+5 \% \mathrm{D}_{2} \mathrm{O}\right): \delta 0.82-0.89(\mathrm{~m}, 18 \mathrm{H}, \mathrm{MeCH}), 0.95(\mathrm{t}, J=7.1 \mathrm{~Hz}$, $\left.84 \mathrm{H}, \quad \mathrm{N}\left(\mathrm{CH}_{2} \mathrm{CH}_{2} \mathrm{CH}_{2} \mathrm{Me}\right)_{4}\right), \quad 1.18 \quad\left(\mathrm{t}, \quad J=7.6 \mathrm{~Hz}, \quad 2 \mathrm{H}, \quad \mathrm{SnCH}_{2}\right), \quad 1.38-1.44 \quad(\mathrm{~m}, \quad 56 \mathrm{H}$, $\left.\mathrm{N}\left(\mathrm{CH}_{2} \mathrm{CH}_{2} \mathrm{CH}_{2} \mathrm{Me}\right)_{4}\right), 1.55-1.70\left(\mathrm{~m}, 56 \mathrm{H}, \mathrm{N}\left(\mathrm{CH}_{2} \mathrm{CH}_{2} \mathrm{CH}_{2} \mathrm{Me}\right)_{4}\right), 2.00-2.28\left(\mathrm{~m}, 5 \mathrm{H}, \mathrm{Me}_{2} \mathrm{CH}+\right.$ $\left.\mathrm{CH}_{2} \mathrm{CH}_{2} \mathrm{CH}_{2}\right), 2.52\left(\mathrm{t}, J=7.6 \mathrm{~Hz}, 2 \mathrm{H}, \mathrm{CH}_{2} \mathrm{C}=\mathrm{O}\right), 2.60\left(\mathrm{~m}, 2 \mathrm{H}, \mathrm{CH}_{2} \mathrm{C}=\mathrm{O}\right), 2.92(\mathrm{t}, J=6.8 \mathrm{~Hz}, 2 \mathrm{H}$, $\left.\mathrm{NHCHCH}_{2}\right)$ 3.08-3.25 (m, $\left.58 \mathrm{H}, \mathrm{N}\left(\mathrm{CH}_{2} \mathrm{CH}_{2} \mathrm{CH}_{2} \mathrm{Me}\right)_{4}+\mathrm{NHCH}_{2} \mathrm{CH}_{2}\right), 4.05$ (bs, $\left.1 \mathrm{H}, \mathrm{NHCH}\right), 4.18$ (m, $2 \mathrm{H}, \mathrm{NHCH}), 4.42\left(\mathrm{t}, J=6.6 \mathrm{~Hz}, 2 \mathrm{H}, \mathrm{CH}_{2} \mathrm{CH}_{2} \mathrm{~N}\right), 7.47(\mathrm{~d}, J=8.8 \mathrm{~Hz}, \mathrm{NH}), 7.51(\mathrm{~d}, J=8.1 \mathrm{~Hz}, \mathrm{NH})$,

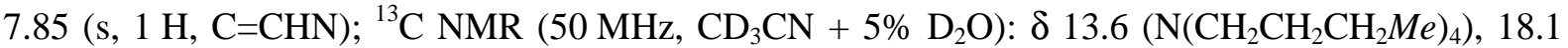
$(\mathrm{MeCH}), 18.4(\mathrm{MeCH}), 18.9(\mathrm{MeCH}), 19.1\left(\mathrm{SnCH}_{2}\right), 19.3(\mathrm{MeCH}), 20.0\left(\mathrm{~N}\left(\mathrm{CH}_{2} \mathrm{CH}_{2} \mathrm{CH}_{2} \mathrm{Me}\right)_{4}\right), 22.1$ $\left(\mathrm{CH}_{2} \mathrm{CH}_{2} \mathrm{CO}\right) 24.1\left(\mathrm{~N}\left(\mathrm{CH}_{2} \mathrm{CH}_{2} \mathrm{CH}_{2} \mathrm{Me}\right)_{4}\right), 30.0\left(\mathrm{CH}_{2} \mathrm{CH}_{2} \mathrm{CH}_{2}\right), 30.6\left(\mathrm{Me}_{2} \mathrm{CH}\right), 30.8\left(\mathrm{Me}_{2} \mathrm{CH}\right), 31.0$ $\left(\mathrm{Me}_{2} \mathrm{CH}\right), 32.0\left(\mathrm{CH}_{2} \mathrm{C}=\mathrm{O}\right), 35.7\left(\mathrm{CH}_{2} \mathrm{C}=\mathrm{O}\right), 36.9\left(\mathrm{NHCH}_{2}\right), 48.4\left(\mathrm{CH}_{2} \mathrm{CH}_{2} \mathrm{~N}\right), 58.4(\mathrm{NHCHC}=\mathrm{O}), 58.9$ $\left(\mathrm{N}\left(\mathrm{CH}_{2} \mathrm{CH}_{2} \mathrm{CH}_{2} \mathrm{Me}\right)_{4}\right), 59.4(\mathrm{NHCHC}=\mathrm{O}), 60.0(\mathrm{NHCHC}=\mathrm{O}), 123.7(\mathrm{C}=\mathrm{CHN}), 146.9(\mathrm{C}=\mathrm{CHN})$, $172.5(\mathrm{C}=\mathrm{O}), 172.8(\mathrm{C}=\mathrm{O}), 173.5(\mathrm{C}=\mathrm{O}), 174.0(\mathrm{C}=\mathrm{O}), 176.1(\mathrm{C}=\mathrm{O}) ;{ }^{31} \mathrm{P}$ NMR $\left(162 \mathrm{MHz}, \mathrm{CD}_{3} \mathrm{CN}+\right.$ $\left.5 \% \mathrm{D}_{2} \mathrm{O}\right): \delta-9.4\left(\mathrm{~s}+\mathrm{d}, 1 \mathrm{P}, J_{S n P}=24.3 \mathrm{~Hz}\right),-12.4(\mathrm{~s}, 1 \mathrm{P})$. 


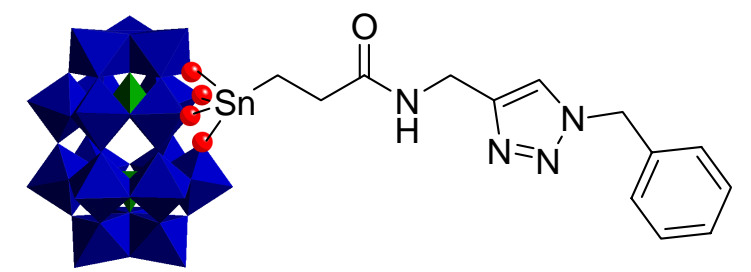

17: $\mathbf{T B A}_{5.8} \mathbf{H}_{1.2}\left[\left(\boldsymbol{\alpha}_{1}\right) \operatorname{Sn}\left(\mathrm{CH}_{2}\right)_{2} \mathrm{CONHCH}_{2}\left(\mathbf{C}_{2} \mathbf{H N}_{3}\right) \mathbf{B n}\right]$. Following GPA, 17 was isolated as a white powder $(127 \mathrm{mg}, 81 \%)$. IR: $\tilde{\boldsymbol{v}}=2961(\mathrm{~m}), 2933(\mathrm{w}), 2872(\mathrm{~m}), 1641(\mathrm{w}), 1484(\mathrm{~m}), 1380(\mathrm{w}), 1083$ (s), 947 (s), 906 (s), 776 (vs) cm ${ }^{-1} ;{ }^{1} \mathrm{H}$ NMR (400 MHz, $\left.\mathrm{CD}_{3} \mathrm{CN}+10 \% \mathrm{D}_{2} \mathrm{O}\right): \delta 0.93(\mathrm{t}, J=7.1 \mathrm{~Hz}$, $\left.84 \mathrm{H}, \mathrm{N}\left(\mathrm{CH}_{2} \mathrm{CH}_{2} \mathrm{CH}_{2} \mathrm{Me}\right)_{4}\right), 1.15-1.18\left(\mathrm{~m}, 2 \mathrm{H}, \mathrm{SnCH}_{2}\right), 1.38-1.44\left(\mathrm{~m}, 56 \mathrm{H}, \mathrm{N}\left(\mathrm{CH}_{2} \mathrm{CH}_{2} \mathrm{CH}_{2} \mathrm{Me}\right)_{4}\right)$, 1.55-1.70 (m, $\left.56 \mathrm{H}, \mathrm{N}\left(\mathrm{CH}_{2} \mathrm{CH}_{2} \mathrm{CH}_{2} \mathrm{Me}\right)_{4}\right), 2.64-2.67\left(\mathrm{~m}, 2 \mathrm{H}, \mathrm{CH}_{2} \mathrm{C}=\mathrm{O}\right), 3.08-3.25(\mathrm{~m}, 56 \mathrm{H}$, $\left.\mathrm{N}\left(\mathrm{CH}_{2} \mathrm{CH}_{2} \mathrm{CH}_{2} \mathrm{Me}\right)_{4}\right), 4.41\left(\mathrm{~s}, 2 \mathrm{H}, \mathrm{NHCH}_{2}\right), 5.50-5.52\left(\mathrm{AB}, 2 \mathrm{H}, \mathrm{CH}_{2} \mathrm{Ph}\right), 7.25-7.38$ (m, $5 \mathrm{H}$, arom.), $7.95(\mathrm{~s}, \quad 1 \mathrm{H}, \quad \mathrm{C}=\mathrm{CHN}) ;{ }^{13} \mathrm{C} \quad \mathrm{NMR}\left(100 \mathrm{MHz}, \mathrm{CD}_{3} \mathrm{CN}\right): \delta \quad 13.9 \quad\left(\mathrm{~N}\left(\mathrm{CH}_{2} \mathrm{CH}_{2} \mathrm{CH}_{2} \mathrm{Me}\right)_{4}\right), \quad 20.3$

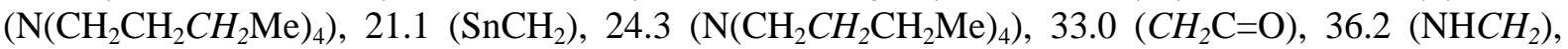
$54.2\left(\mathrm{CH}_{2} \mathrm{Ph}\right), 59.1\left(\mathrm{~N}\left(\mathrm{CH}_{2} \mathrm{CH}_{2} \mathrm{CH}_{2} \mathrm{Me}\right)_{4}\right), 124.3(\mathrm{C}=\mathrm{CHN}), 128.8(\mathrm{CH}$ arom. $), 129.5(\mathrm{CH}$ arom.), 129.6 (CH arom.), 137.4 (C arom.), $147.4(C=\mathrm{CHN}), 175.8(\mathrm{C}=\mathrm{O}) ;{ }^{31} \mathrm{P}$ NMR $\left(162 \mathrm{MHz}, \mathrm{CD}_{3} \mathrm{CN}+\right.$ $\left.10 \% \quad \mathrm{D}_{2} \mathrm{O}\right): \delta-6.6\left(\mathrm{~s}+\mathrm{d}, \quad 1 \mathrm{P}, \quad J_{S n P}=40.5 \mathrm{~Hz}\right),-11.9 \quad(\mathrm{~s}, \quad 1 \mathrm{P})$; elemental analysis $(\%)$ for $\mathrm{C}_{105.8} \mathrm{H}_{225} \mathrm{~N}_{9.8} \mathrm{O}_{62} \mathrm{P}_{2} \mathrm{SnW}_{17}\left(5932.88 \mathrm{~g} \cdot \mathrm{mol}^{-1}\right)$ : calc. C 21.42, H 3.82, N 2.31; found C 21.39, H 3.79, $\mathrm{N} 2.14$.

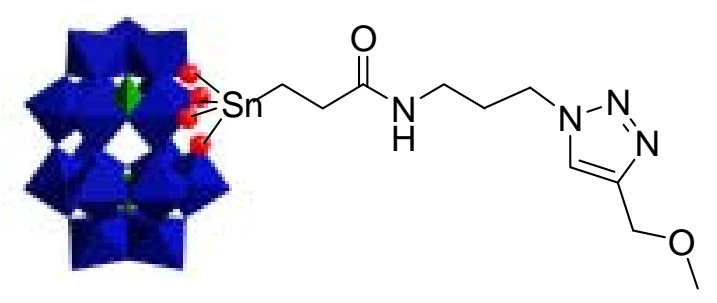

18: $\mathrm{TBA}_{6.6} \mathrm{H}_{0.4}\left[\left(\boldsymbol{\alpha}_{1}\right) \mathrm{Sn}\left(\mathrm{CH}_{2}\right)_{2} \mathbf{C O N H}\left(\mathrm{CH}_{2}\right)_{3}\left(\mathbf{N}_{3} \mathrm{C}_{2} \mathrm{H}\right) \mathrm{CH}_{2} \mathrm{OMe}\right]$. Following GPA, 18 was isolated as a white powder (111 mg, $72 \%)$. IR: $\tilde{\boldsymbol{v}}=2960(\mathrm{~m}), 2933(\mathrm{w}), 2872(\mathrm{~m}), 1641(\mathrm{w}), 1483(\mathrm{~m}), 1379(\mathrm{w})$, 1082 (s), 946 (s), 905 (s), 772 (vs) $\mathrm{cm}^{-1} ;{ }^{1} \mathrm{H}$ NMR (400 MHz, $\mathrm{CD}_{3} \mathrm{CN}$ ): $\delta$ 0.80-1.20 (m, $81 \mathrm{H}$, $\left.\mathrm{N}\left(\mathrm{CH}_{2} \mathrm{CH}_{2} \mathrm{CH}_{2} \mathrm{Me}\right)_{4}+\mathrm{SnCH}_{2}\right), 1.38-1.44\left(\mathrm{~m}, 53 \mathrm{H}, \mathrm{N}\left(\mathrm{CH}_{2} \mathrm{CH}_{2} \mathrm{CH}_{2} \mathrm{Me}\right)_{4}\right), 1.55-1.70$ (m, $53 \mathrm{H}$, $\left.\mathrm{N}\left(\mathrm{CH}_{2} \mathrm{CH}_{2} \mathrm{CH}_{2} \mathrm{Me}\right)_{4}\right), 2.17-2.20\left(\mathrm{~m}, 2 \mathrm{H}, \mathrm{CH}_{2} \mathrm{CH}_{2} \mathrm{CH}_{2}\right), 2.60-2.78\left(\mathrm{~m}, 2 \mathrm{H}, \mathrm{CH}_{2} \mathrm{C}=\mathrm{O}\right), 3.08-3.25(\mathrm{~m}$, $\left.55 \mathrm{H}, \mathrm{N}\left(\mathrm{CH}_{2} \mathrm{CH}_{2} \mathrm{CH}_{2} \mathrm{Me}\right)_{4}+\mathrm{NHCH}_{2} \mathrm{CH}_{2}\right), 3.31(\mathrm{~s}, 3 \mathrm{H}, \mathrm{OMe}), 4.46\left(\mathrm{~s}, 2 \mathrm{H}, \mathrm{OCH}_{2}\right), 4.52-4.58(\mathrm{~m}$, $\left.2 \mathrm{H}, \mathrm{CH}_{2} \mathrm{~N}\right), 7.49(\mathrm{~s}, 1 \mathrm{H}, \mathrm{NH}) 8.03(\mathrm{~s}, 1 \mathrm{H}, \mathrm{C}=\mathrm{CHN}) ;{ }^{13} \mathrm{C} \mathrm{NMR}\left(50 \mathrm{MHz}, \mathrm{CD}_{3} \mathrm{CN}\right): \delta 13.9$ $\left(\mathrm{N}\left(\mathrm{CH}_{2} \mathrm{CH}_{2} \mathrm{CH}_{2} \mathrm{Me}\right)_{4}\right), 20.3\left(\mathrm{~N}\left(\mathrm{CH}_{2} \mathrm{CH}_{2} \mathrm{CH}_{2} \mathrm{Me}\right)_{4}\right), 22.7\left(\mathrm{SnCH}_{2}\right), 24.3\left(\mathrm{~N}\left(\mathrm{CH}_{2} \mathrm{CH}_{2} \mathrm{CH}_{2} \mathrm{Me}\right)_{4}\right), 30.6$ $\left(\mathrm{CH}_{2} \mathrm{CH}_{2} \mathrm{CH}_{2}\right), 33.2\left(\mathrm{CH}_{2} \mathrm{C}=\mathrm{O}\right), 36.5\left(\mathrm{NHCH}_{2}\right), 48.5\left(\mathrm{CH}_{2} \mathrm{~N}\right), 57.9(\mathrm{MeO}), 59.0\left(\mathrm{~N}\left(\mathrm{CH}_{2} \mathrm{Pr}\right)_{4}\right), 66.1$ $\left(\mathrm{OCH}_{2}\right), 125.8(\mathrm{C}=C H \mathrm{~N}), 144.3(\mathrm{C}=\mathrm{CHN}), 175.8(\mathrm{C}=\mathrm{O}) ;{ }^{31} \mathrm{P}$ NMR $\left(162 \mathrm{MHz}, \mathrm{CD}_{3} \mathrm{CN}\right): \delta-6.6(\mathrm{~s}+\mathrm{d}$, $\left.1 \mathrm{P}, J_{S n P}=40.5 \mathrm{~Hz}\right),-12.2(\mathrm{~s}, 1 \mathrm{P})$; elemental analysis $(\%)$ for $\mathrm{C}_{115.6} \mathrm{H}_{255} \mathrm{~N}_{10.6} \mathrm{O}_{63} \mathrm{P}_{2} \mathrm{SnW}_{17}(6108.03$ $\mathrm{g} \cdot \mathrm{mol}^{-1}$ ): calc. C 22.73, H $4.21 \mathrm{~N} 2.43$; found C 22.85, H 4.31, N 2.21. 


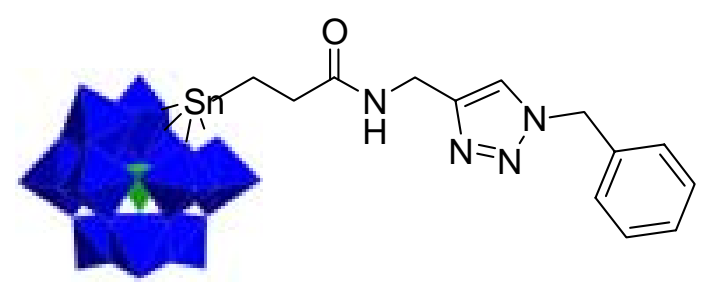

19: $\operatorname{TBA}_{4}\left[(\mathrm{~K}) \operatorname{Sn}\left(\mathrm{CH}_{2}\right)_{2} \mathrm{CONHCH}_{2}\left(\mathrm{C}_{2} \mathrm{HN}_{3}\right) \mathrm{Bn}\right]$. Following GPB, 19 was isolated as a white powder (114 mg, $71 \%)$. IR: $\tilde{v}=2961(\mathrm{~m}), 2934(\mathrm{w}), 2873(\mathrm{~m}), 1664(\mathrm{w}), 1483(\mathrm{~m}), 1379(\mathrm{w}), 1065$ (s), 960 (s), 882 (s), 796 (vs) $\mathrm{cm}^{-1} ;{ }^{1} \mathrm{H}$ NMR $\left(400 \mathrm{MHz}, \mathrm{CD}_{3} \mathrm{CN}\right): \delta 0.98 \quad(\mathrm{t}, \quad J=7.1 \mathrm{~Hz}, 48 \mathrm{H}$, $\left.\mathrm{N}\left(\mathrm{CH}_{2} \mathrm{CH}_{2} \mathrm{CH}_{2} \mathrm{Me}\right)_{4}\right), 1.38-1.44\left(\mathrm{~m}, 34 \mathrm{H}, \mathrm{N}\left(\mathrm{CH}_{2} \mathrm{CH}_{2} \mathrm{CH}_{2} \mathrm{Me}\right)_{4}+\mathrm{SnCH}_{2}\right), 1.55-1.70(\mathrm{~m}, 32 \mathrm{H}$, $\left.\mathrm{N}\left(\mathrm{CH}_{2} \mathrm{CH}_{2} \mathrm{CH}_{2} \mathrm{Me}\right)_{4}\right), 2.54\left(\mathrm{t}, J=7.8 \mathrm{~Hz}, 2 \mathrm{H}, \mathrm{CH}_{2} \mathrm{C}=\mathrm{O}\right), 3.08-3.25\left(\mathrm{~m}, 32 \mathrm{H}, \mathrm{N}\left(\mathrm{CH}_{2} \mathrm{Pr}\right)_{4}\right), 4.40$ (d, $J=5.2 \mathrm{~Hz}, 2 \mathrm{H}, \mathrm{NHCH}_{2}$ ), 5.53 (s, $2 \mathrm{H}, \mathrm{CH}_{2} \mathrm{Ph}$ ), 7.03 (bs, $1 \mathrm{H}, \mathrm{NH}$ ), 7.30-7.39 (m, $5 \mathrm{H}$ arom.), 7.83 (s,

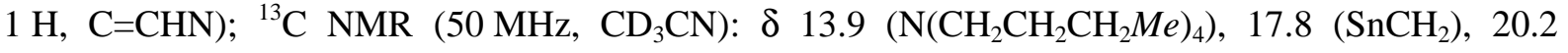

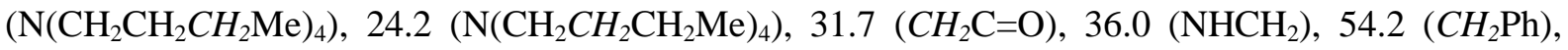

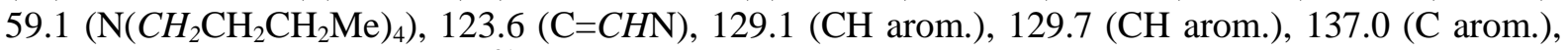
$146.9(C=\mathrm{CHN}), 173.9(\mathrm{C}=\mathrm{O}) ;{ }^{31} \mathrm{P}$ NMR $\left(162 \mathrm{MHz}, \mathrm{CD}_{3} \mathrm{CN}\right): \delta-10.9\left(\mathrm{~s}+\mathrm{d}, 1 \mathrm{P}, J_{S n P}=19.4 \mathrm{~Hz}\right)$; $\mathrm{ESI}^{-}$ /MS: see full details below.

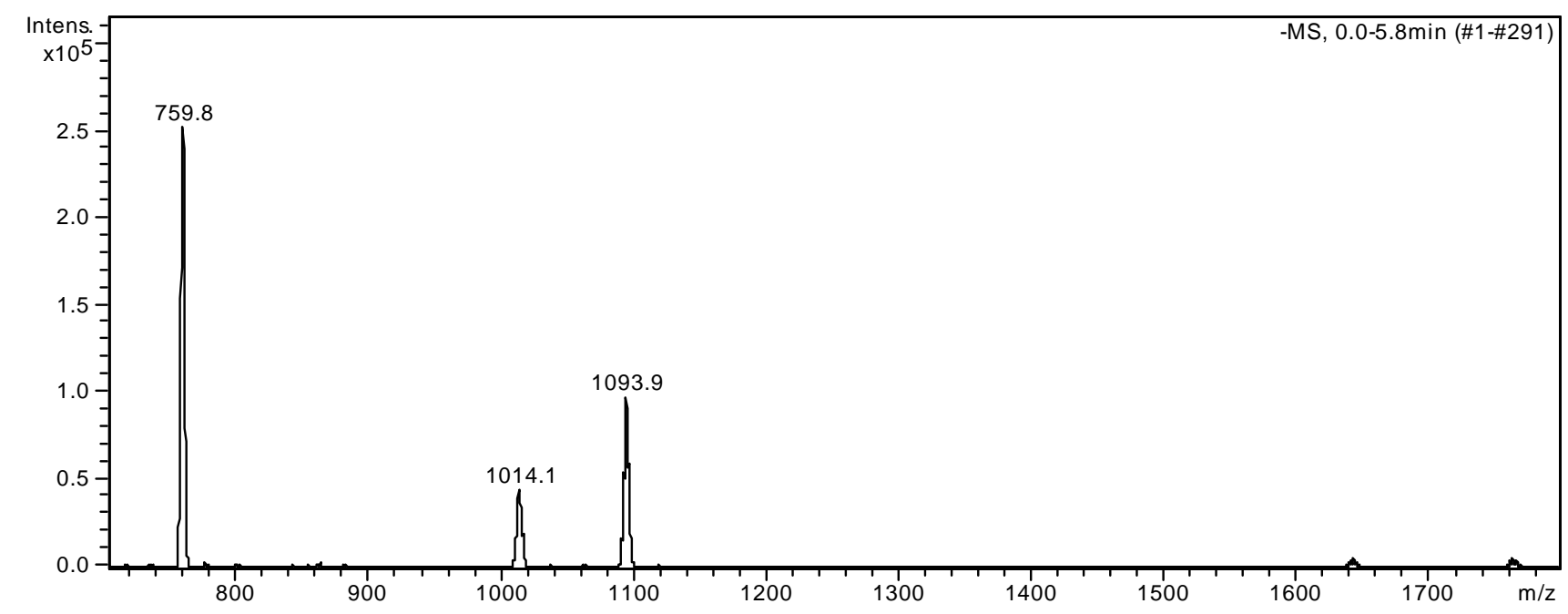

\begin{tabular}{cccccc}
\hline Entry & Charge & $\begin{array}{c}\text { Simulated } \\
\mathrm{m} / \mathrm{z}\end{array}$ & $\begin{array}{c}\text { Observed } \\
\mathrm{m} / \mathrm{z}\end{array}$ & $\begin{array}{c}\text { Relative } \\
\text { intensity }\end{array}$ & Composition \\
\hline 1 & $2-$ & 1761.9 & 1760.9 & 1.8 & $\mathrm{TBA}_{2}\left[(\mathrm{~K}) \mathrm{Sn}\left(\mathrm{CH}_{2}\right)_{2} \mathrm{CONHCH}_{2}\left(\mathrm{C}_{2} \mathrm{HN}_{3}\right) \mathrm{Bn}\right]$ \\
2 & $2-$ & 1641.3 & 1641.2 & 1.9 & $\mathrm{TBAH}\left[(\mathrm{K}) \operatorname{Sn}\left(\mathrm{CH}_{2}\right)_{2} \mathrm{CONHCH}_{2}\left(\mathrm{C}_{2} \mathrm{HN}_{3}\right) \mathrm{Bn}\right]$ \\
3 & $3-$ & 1093.8 & 1093.9 & 38.5 & $\mathrm{TBA}\left[(\mathrm{K}) \mathrm{Sn}\left(\mathrm{CH}_{2}\right)_{2} \mathrm{CONHCH}_{2}\left(\mathrm{C}_{2} \mathrm{HN}_{3}\right) \mathrm{Bn}\right]$ \\
4 & $3-$ & 1013.4 & 1013.2 & 17.3 & $\mathrm{H}\left[(\mathrm{K}) \mathrm{Sn}\left(\mathrm{CH}_{2}\right)_{2} \mathrm{CONHCH}_{2}\left(\mathrm{C}_{2} \mathrm{HN}_{3}\right) \mathrm{Bn}\right]$ \\
5 & $4-$ & 759.8 & 759.8 & 100 & {$\left[(\mathrm{~K}) \mathrm{Sn}\left(\mathrm{CH}_{2}\right)_{2} \mathrm{CONHCH}_{2}\left(\mathrm{C}_{2} \mathrm{HN}_{3}\right) \mathrm{Bn}\right]$} \\
\hline
\end{tabular}




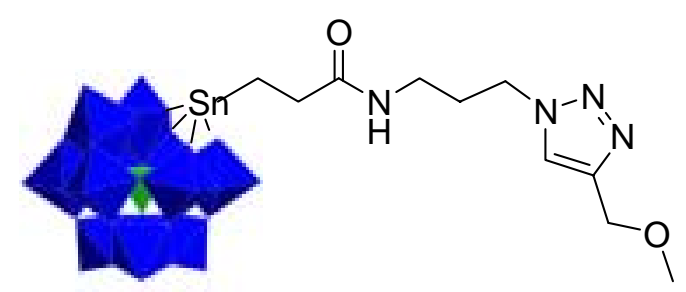

20: $\mathbf{T B A}_{4}\left[(\mathrm{~K}) \mathrm{Sn}\left(\mathrm{CH}_{2}\right)_{2} \mathbf{C O N H}\left(\mathrm{CH}_{2}\right)_{3}\left(\mathbf{N}_{3} \mathbf{C}_{2} \mathbf{H}\right) \mathrm{CH}_{2} \mathrm{OMe}\right]$. Following GPB, 20 was isolated as a white powder (100 mg, $63 \%)$. IR: $\tilde{\boldsymbol{V}}=2961(\mathrm{~m}), 2934(\mathrm{w}), 2874(\mathrm{~m}), 1663(\mathrm{w}), 1483(\mathrm{~m}), 1380(\mathrm{w}), 1066$ (s), 960 (s), 883 (s), 800 (vs) cm ${ }^{-1}$; ${ }^{1} \mathrm{H}$ NMR (400 MHz, CD ${ }_{3} \mathrm{CN}$ ): $\delta 0.98$ (t, $J=7.1 \mathrm{~Hz}, 48 \mathrm{H}$, $\left.\mathrm{N}\left(\mathrm{CH}_{2} \mathrm{CH}_{2} \mathrm{CH}_{2} \mathrm{Me}\right)_{4}\right)$, 1.38-1.44 (m, $\left.34 \mathrm{H}, \mathrm{N}\left(\mathrm{CH}_{2} \mathrm{CH}_{2} \mathrm{CH}_{2} \mathrm{Me}\right)_{4}+\mathrm{SnCH}_{2}\right), 1.55-1.70$ (m, $32 \mathrm{H}$, $\left.\mathrm{N}\left(\mathrm{CH}_{2} \mathrm{CH}_{2} \mathrm{CH}_{2} \mathrm{Me}\right)_{4}\right), 2.08-2.12\left(\mathrm{~m}, 2 \mathrm{H}, \mathrm{CH}_{2} \mathrm{CH}_{2} \mathrm{CH}_{2}\right), 2.52$ (t, $\left.J=7.6 \mathrm{~Hz}, 2 \mathrm{H}, \mathrm{CH}_{2} \mathrm{C}=\mathrm{O}\right), 3.08-3.25$ $\left(\mathrm{m}, 34 \mathrm{H}, \mathrm{N}\left(\mathrm{CH}_{2} \mathrm{CH}_{2} \mathrm{CH}_{2} \mathrm{Me}\right)_{4}+\mathrm{NHCH}_{2}\right), 3.34(\mathrm{~s}, 3 \mathrm{H}, \mathrm{OMe}), 4.42\left(\mathrm{t}, J=6.8 \mathrm{~Hz}, 2 \mathrm{H}, \mathrm{CH}_{2} \mathrm{~N}\right), 4.47$ $\left(\mathrm{s}, 2 \mathrm{H}, \mathrm{OCH}_{2}\right), 6.75(\mathrm{t}, J=5.2 \mathrm{~Hz}, 1 \mathrm{H}, \mathrm{NH}), 7.92(\mathrm{~s}, 1 \mathrm{H}, \mathrm{C}=\mathrm{CHN}) ;{ }^{13} \mathrm{C} \mathrm{NMR}\left(100 \mathrm{MHz}, \mathrm{CD}_{3} \mathrm{CN}\right)$ :

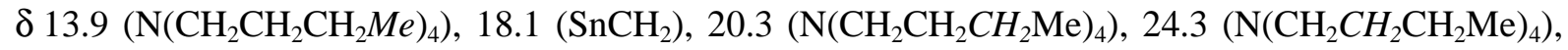
$30.9 \quad\left(\mathrm{CH}_{2} \mathrm{CH}_{2} \mathrm{CH}_{2}\right), \quad 32.0 \quad\left(\mathrm{CH}_{2} \mathrm{C}=\mathrm{O}\right), \quad 36.9 \quad\left(\mathrm{NHCH}_{2}\right), \quad 48.4 \quad\left(\mathrm{CH}_{2} \mathrm{~N}\right), \quad 58.0 \quad(\mathrm{MeO}), \quad 59.1$ $\left(\mathrm{N}\left(\mathrm{CH}_{2} \mathrm{CH}_{2} \mathrm{CH}_{2} \mathrm{Me}\right)_{4}\right), 66.1\left(\mathrm{OCH}_{2}\right), 125.1(\mathrm{C}=\mathrm{CHN}), 145.1(\mathrm{C}=\mathrm{CHN}), 174.2(\mathrm{C}=\mathrm{O}) ;{ }^{31} \mathrm{P} \mathrm{NMR}$ $\left(162 \mathrm{MHz}, \quad \mathrm{CD}_{3} \mathrm{CN}\right): \quad \delta-10.9 \quad\left(\mathrm{~s}+\mathrm{d}, \quad 1 \mathrm{P}, \quad J_{S n P}=17.8 \mathrm{~Hz}\right) ; \quad$ elemental analysis $(\%)$ for $\mathrm{C}_{74} \mathrm{H}_{161} \mathrm{~N}_{8} \mathrm{O}_{41} \mathrm{PSnW}{ }_{11}\left(3991.15 \mathrm{~g} \cdot \mathrm{mol}^{-1}\right)$ : calc. C 22.27, H 4.07, N 2.81; found C 22.53, H 3.91, N 2.45.

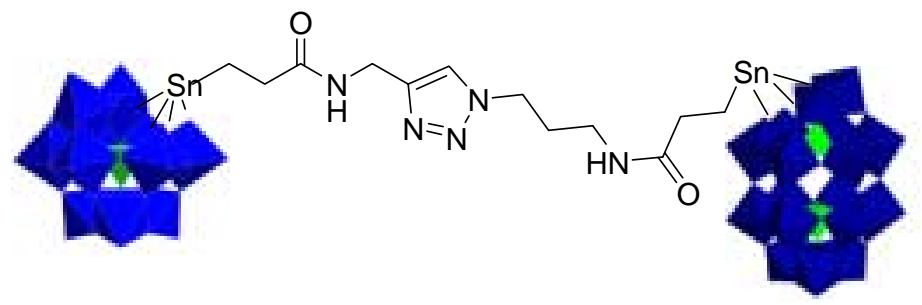

21: $\mathrm{TBA}_{10.5} \mathrm{H}_{0.5}\left[(\mathrm{~K}) \mathrm{Sn}\left(\mathrm{CH}_{2}\right)_{2} \mathrm{CONHCH}_{2}\left(\mathrm{C}_{2} \mathrm{HN}_{3}\right)\left(\mathrm{CH}_{2}\right)_{3} \mathrm{NHCO}\left(\mathrm{CH}_{2}\right)_{2} \mathrm{Sn}\left(\boldsymbol{\alpha}_{2}\right)\right]$. To a solution of $\mathrm{TBA}_{5}\left[\mathrm{Et}_{3} \mathrm{NH}^{+}\right]_{2}\left[\mathrm{P}_{2} \mathrm{~W}_{17} \mathrm{O}_{61}\left\{\mathrm{SnCH}_{2} \mathrm{CH}_{2} \mathrm{C}(=\mathrm{O}) \mathrm{NH}\left(\mathrm{CH}_{2}\right)_{3} \mathrm{~N}_{3}\right\}\right] \quad(0.026 \mathrm{mmol}, 150 \mathrm{mg})$ and $\mathrm{TBA}_{3}\left[\mathrm{Et}_{3} \mathrm{NH}^{+}\right]\left[\mathrm{PW}_{11} \mathrm{O}_{39}\left\{\mathrm{SnCH}_{2} \mathrm{CH}_{2} \mathrm{C}(=\mathrm{O}) \mathrm{NHCH}_{2} \mathrm{CCH}\right\}\right]$ (0.039 mmol, $147 \mathrm{mg}, 1.5$ equiv.) in acetonitrile $(1 \mathrm{~mL})$ were added a solution of $\mathrm{CuSO}_{4} \cdot 5 \mathrm{H}_{2} \mathrm{O}(0.13 \mathrm{mmol}, 5$ equiv., $33 \mathrm{mg})$ in water $(1 \mathrm{~mL})$ and a solution of sodium ascorbate $(1.3 \mathrm{mmol}, 50$ equiv., $257 \mathrm{mg})$ in water $(1 \mathrm{~mL})$. The mixture was left at room temperature for 4 days. Cation-exchange resin beads (Amberlyst, 16-50 mesh, $\mathrm{TBA}^{+}$form) were then added and the reaction mixture was stirred for 1 hour. Acetone $(10 \mathrm{~mL})$ was then added. If no precipitate appeared, the resin could be filtered off and the filtrate was concentrated in vacuo. If a precipitate appeared, the mixture was left stirring until its disappeareance. Dichloromethane $(30 \mathrm{~mL})$ and water $(30 \mathrm{~mL})$ were added to the residue. The insoluble excess Keggin reagent was filtered off. After a first extraction, the organic phase was washed twice with water $(30 \mathrm{~mL})$, then concentrated in vасио. The white solid which was obtained was dissolved in acetone $(2 \mathrm{~mL})$ and precipitated in $\mathrm{EtOH} / \mathrm{Et}_{2} \mathrm{O}(2 \mathrm{~mL} / 30 \mathrm{~mL})$. The solid was isolated by centrifugation, washed with $\mathrm{Et}_{2} \mathrm{O}$ and dried in vacuo to afford 21 as a white powder (150 mg, $59 \%)$.

IR: $\tilde{\boldsymbol{v}}=2960(\mathrm{~m}), 2934(\mathrm{w}), 2872(\mathrm{~m}), 1655(\mathrm{w}), 1483(\mathrm{~m}), 1379(\mathrm{w}), 1089(\mathrm{~s}), 1066(\mathrm{~s}), 953(\mathrm{~s}), 885$ (s), 780 (vs) $\mathrm{cm}^{-1} ;{ }^{1} \mathrm{H}$ NMR $\left(400 \mathrm{MHz}, \mathrm{CD}_{3} \mathrm{CN}+10 \% \mathrm{D}_{2} \mathrm{O}\right): \delta 0.98(\mathrm{t}, J=7.1 \mathrm{~Hz}, 134 \mathrm{H}$, $\mathrm{N}\left(\mathrm{CH}_{2} \mathrm{CH}_{2} \mathrm{CH}_{2} \mathrm{Me}\right)_{4}[\mathrm{D}+\mathrm{K}]+\mathrm{SnCH}_{2}$ [Dawson]), 1.38-1.44 (m, $90 \mathrm{H}, \mathrm{N}\left(\mathrm{CH}_{2} \mathrm{CH}_{2} \mathrm{CH}_{2} \mathrm{Me}\right)_{4}[\mathrm{D}+\mathrm{K}]+$ $\mathrm{SnCH}_{2}$ [Keggin]), 1.55-1.70 (m, $\left.88 \mathrm{H}, \mathrm{N}\left(\mathrm{CH}_{2} \mathrm{CH}_{2} \mathrm{CH}_{2} \mathrm{Me}\right)_{4}[\mathrm{D}+\mathrm{K}]\right)$, 2.04-2.08 (m, $2 \mathrm{H}, \mathrm{CH}_{2} \mathrm{CH}_{2} \mathrm{CH}_{2}$ ), $2.50\left(\mathrm{t}, J=8.6 \mathrm{~Hz}, 2 \mathrm{H}\right.$, one $\left.\mathrm{CH}_{2} \mathrm{C}=\mathrm{O}\right), 2.52\left(\mathrm{t}, J=8.6 \mathrm{~Hz}, 2 \mathrm{H}\right.$, one $\left.\mathrm{CH}_{2} \mathrm{C}=\mathrm{O}\right), 3.08-3.25(\mathrm{~m}, 90 \mathrm{H}$, $\left.\mathrm{N}\left(\mathrm{CH}_{2} \mathrm{Pr}\right)_{4}[\mathrm{D}+\mathrm{K}]+\mathrm{CH}_{2} \mathrm{CH}_{2} \mathrm{NH}\right), 4.38\left(\mathrm{~m}, 4 \mathrm{H}, \mathrm{ArCH}_{2}+\mathrm{NCH}_{2}\right), 7.82(\mathrm{~s}, 1 \mathrm{H}, \mathrm{C}=\mathrm{CHN}) ;{ }^{13} \mathrm{C} \mathrm{NMR}$

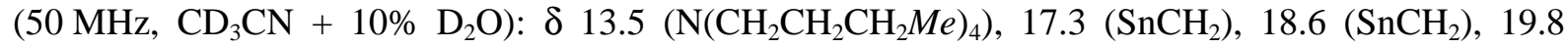
$\left(\mathrm{N}\left(\mathrm{CH}_{2} \mathrm{CH}_{2} \mathrm{CH}_{2} \mathrm{Me}\right)_{4}\right), 23.8 \quad\left(\mathrm{~N}\left(\mathrm{CH}_{2} \mathrm{CH}_{2} \mathrm{CH}_{2} \mathrm{Me}\right)_{4}\right), 30.0 \quad\left(\mathrm{CH}_{2} \mathrm{CH}_{2} \mathrm{CH}_{2}\right), \quad 31.0 \quad\left(\mathrm{CH}_{2} \mathrm{C}=\mathrm{O}\right), \quad 31.5$ 
$\left(\mathrm{CH}_{2} \mathrm{C}=\mathrm{O}\right), 35.0\left(\mathrm{NHCH}_{2}\right), 36.6\left(\mathrm{NHCH}_{2}\right), 48.2\left(\mathrm{NCH}_{2}\right), 58.6\left(\mathrm{~N}\left(\mathrm{CH}_{2} \mathrm{CH}_{2} \mathrm{CH}_{2} \mathrm{Me}\right)_{4}\right), 124.0(\mathrm{C}=\mathrm{CHN})$, 145.2 ( $C=\mathrm{CHN}), 174.7(\mathrm{C}=\mathrm{O}), 176.3(\mathrm{C}=\mathrm{O}) ;{ }^{31} \mathrm{P} \mathrm{NMR}\left(162 \mathrm{MHz}, \mathrm{CD}_{3} \mathrm{CN}+10 \% \mathrm{D}_{2} \mathrm{O}, \mathrm{D}_{1}=40 \mathrm{~s}\right): \delta-$ $9.4\left(\mathrm{~s}+\mathrm{d}, 1 \mathrm{P}, J_{S n P}=27.5 \mathrm{~Hz}, \mathrm{D}\right),-10.9\left(\mathrm{~s}+\mathrm{d}, 1 \mathrm{P}, J_{S n P}=17.8 \mathrm{~Hz}, \mathrm{~K}\right),-12.4(\mathrm{~s}, 1 \mathrm{P}, \mathrm{D})$; elemental analysis (\%) for $\mathrm{C}_{180} \mathrm{H}_{397.5} \mathrm{~N}_{15.5} \mathrm{O}_{102} \mathrm{P}_{3} \mathrm{Sn}_{2} \mathrm{~W}_{28}\left(9889.8 \mathrm{~g} \cdot \mathrm{mol}^{-1}\right)$ : calc. C 21.86, H 4.05, N 2.20, P 0.94, W 52.05, Sn 2.40; found C 21.90, H 3.80, N 2.28, P 0.92, W 48.89, Sn 2.26; ESI/MS: see below for full details.

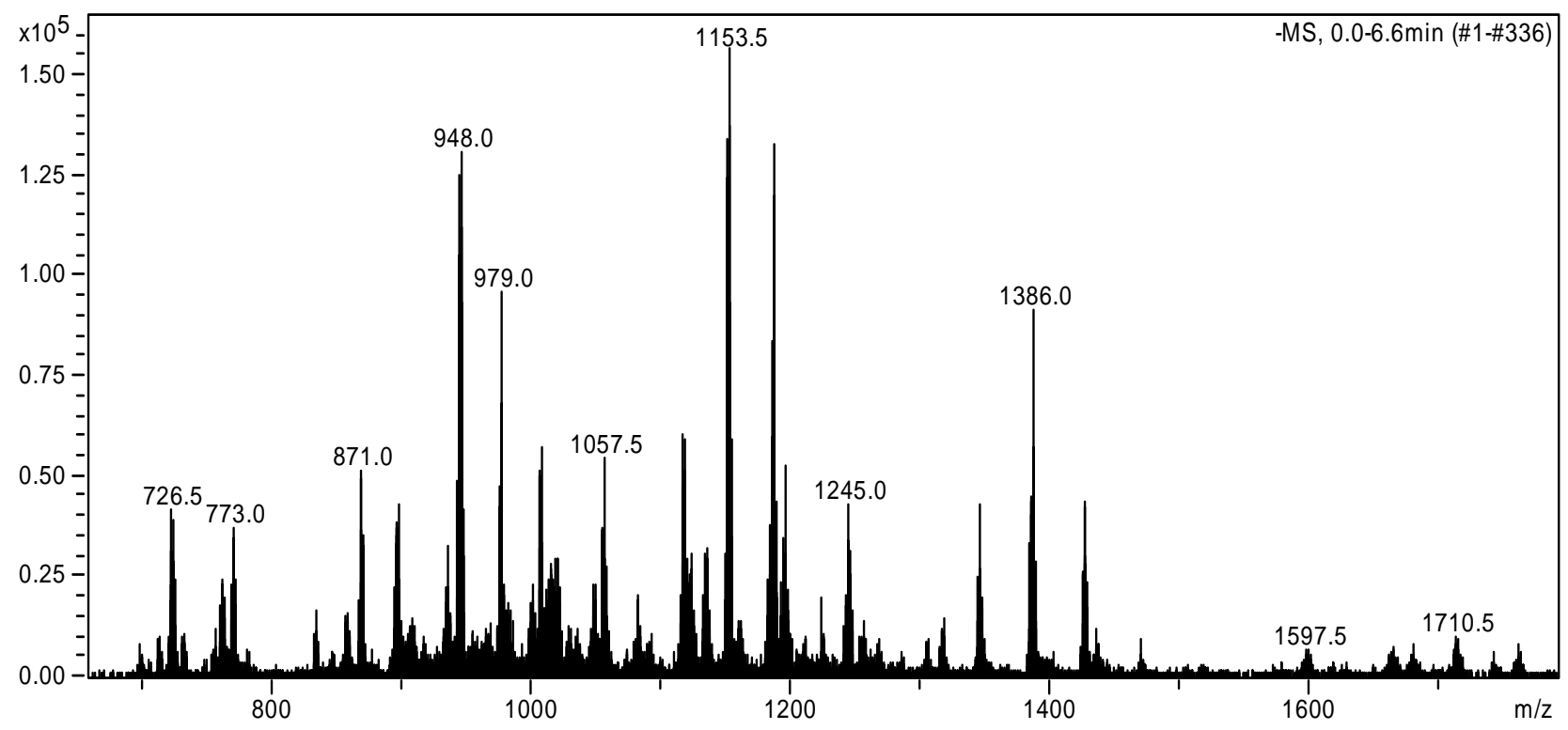

\begin{tabular}{|c|c|c|c|c|c|}
\hline Entry & Charge & $\begin{array}{c}\text { Simulated } \\
\mathrm{m} / \mathrm{z}\end{array}$ & $\begin{array}{c}\text { Observed } \\
\mathrm{m} / \mathrm{z}\end{array}$ & $\begin{array}{l}\text { Relative } \\
\text { intensity }\end{array}$ & Composition \\
\hline 1 & $5-$ & 1759.7 & 1758.9 & 4.2 & $\mathrm{TBA}_{6}\left[(\mathrm{~K}) \mathrm{Sn}----\operatorname{Sn}\left(\alpha_{2}\right)\right]$ \\
\hline 2 & $5-$ & 1711.3 & 1710.5 & 6.3 & $\mathrm{TBA}_{5} \mathrm{H}\left[(\mathrm{K}) \mathrm{Sn}----\mathrm{Sn}\left(\alpha_{2}\right)\right]$ \\
\hline 3 & $5-$ & 1663.0 & 1663.1 & 4.0 & $\mathrm{TBA}_{4} \mathrm{H}_{2}\left[(\mathrm{~K}) \mathrm{Sn}----\mathrm{Sn}\left(\alpha_{2}\right)\right]$ \\
\hline 4 & $6-$ & 1425.9 & 1425.3 & 24.7 & $\mathrm{TBA}_{5}\left[(\mathrm{~K}) \mathrm{Sn}----\operatorname{Sn}\left(\alpha_{2}\right)\right]$ \\
\hline 5 & $6-$ & 1385.7 & 1386.0 & 47.6 & $\mathrm{TBA}_{4} \mathrm{H}\left[(\mathrm{K}) \mathrm{Sn}----\mathrm{Sn}\left(\alpha_{2}\right)\right]$ \\
\hline 6 & $6-$ & 1345.4 & 1345.5 & 22.0 & $\mathrm{TBA}_{3} \mathrm{H}_{2}\left[(\mathrm{~K}) \mathrm{Sn}----\mathrm{Sn}\left(\alpha_{2}\right)\right]$ \\
\hline 7 & 7- & 1187.6 & 1187.5 & 68.7 & $\mathrm{TBA}_{4}\left[(\mathrm{~K}) \mathrm{Sn}----\operatorname{Sn}\left(\alpha_{2}\right)\right]$ \\
\hline 8 & $7-$ & 1153.1 & 1153.5 & 100 & $\mathrm{TBA}_{3} \mathrm{H}\left[(\mathrm{K}) \mathrm{Sn}----\mathrm{Sn}\left(\alpha_{2}\right)\right]$ \\
\hline 9 & 7- & 1118.5 & 1118.6 & 34.6 & $\mathrm{TBA}_{2} \mathrm{H}_{2}\left[(\mathrm{~K}) \mathrm{Sn}----\mathrm{Sn}\left(\alpha_{2}\right)\right]$ \\
\hline 10 & $8-$ & 1008.9 & 1009.3 & 32.6 & $\mathrm{TBA}_{3}\left[(\mathrm{~K}) \mathrm{Sn}----\mathrm{Sn}\left(\alpha_{2}\right)\right]$ \\
\hline 11 & $8-$ & 978.6 & 979.0 & 46.7 & $\mathrm{TBA}_{2} \mathrm{H}\left[(\mathrm{K}) \mathrm{Sn}----\mathrm{Sn}\left(\alpha_{2}\right)\right]$ \\
\hline 12 & $8-$ & 948.5 & 948.0 & 81.0 & $\mathrm{TBAH}_{2}\left[(\mathrm{~K}) \mathrm{Sn}----\mathrm{Sn}\left(\alpha_{2}\right)\right]$ \\
\hline 13 & 9- & 869.8 & 871.0 & 32.5 & $\mathrm{TBA}_{2}\left[(\mathrm{~K}) \mathrm{Sn}----\operatorname{Sn}\left(\alpha_{2}\right)\right]$ \\
\hline
\end{tabular}




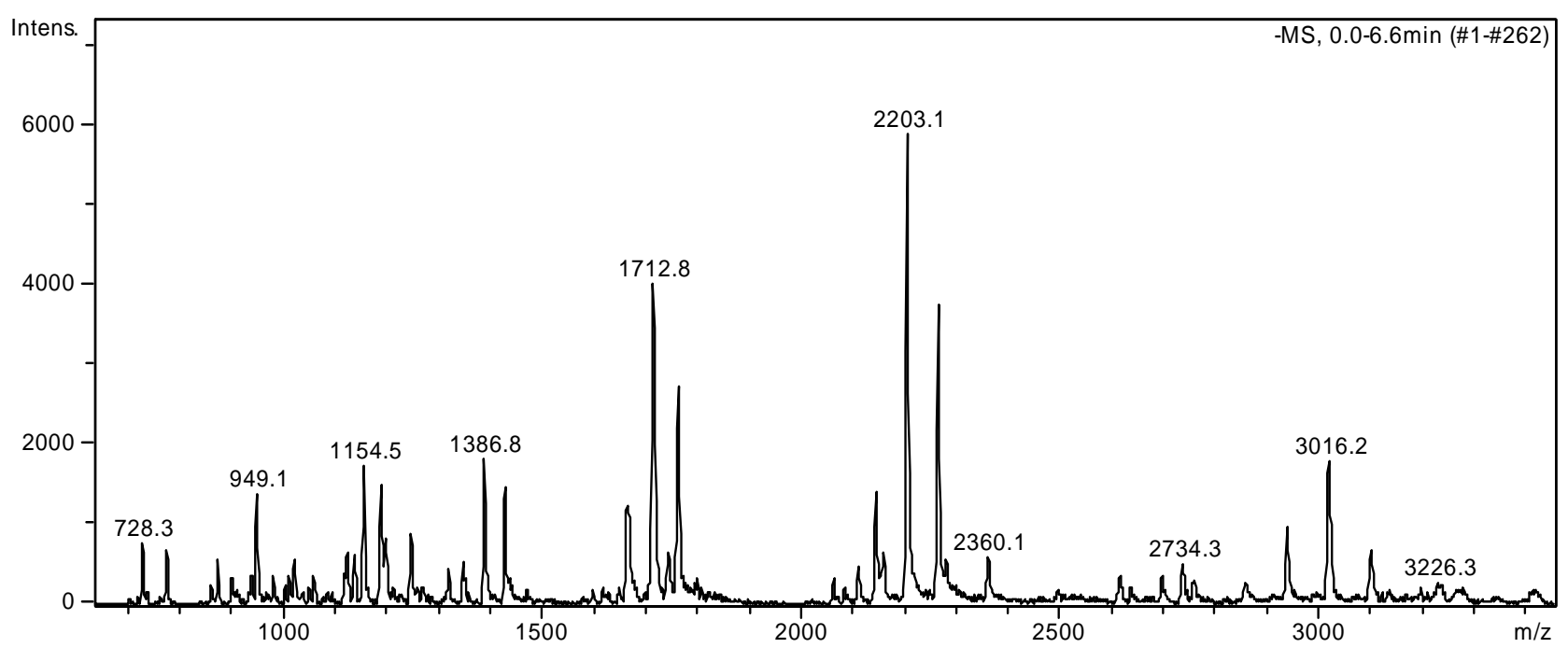

\begin{tabular}{|c|c|c|c|c|c|}
\hline Entry & Charge & $\begin{array}{c}\text { Simulated } \\
\mathrm{m} / \mathrm{z}\end{array}$ & $\begin{array}{c}\text { Observed } \\
\mathrm{m} / \mathrm{z}\end{array}$ & $\begin{array}{l}\text { Relative } \\
\text { intensity }\end{array}$ & Composition \\
\hline 1 & $3-$ & 3094.5 & 3097.7 & 11.9 & $\mathrm{TBA}_{8}\left[(\mathrm{~K}) \mathrm{Sn}----\operatorname{Sn}\left(\alpha_{2}\right)\right]$ \\
\hline 2 & $3-$ & 3013.6 & 3016.2 & 30.4 & $\mathrm{TBA}_{7} \mathrm{H}\left[(\mathrm{K}) \mathrm{Sn}----\operatorname{Sn}\left(\alpha_{2}\right)\right]$ \\
\hline 3 & $3-$ & 2933.2 & 2935.7 & 16.8 & $\mathrm{TBA}_{6} \mathrm{H}_{2}\left[(\mathrm{~K}) \mathrm{Sn}\right.$----Sn($\left.\left(\alpha_{2}\right)\right]$ \\
\hline 4 & $4-$ & 2260.1 & 2263.2 & 63.8 & $\mathrm{TBA}_{7}\left[(\mathrm{~K}) \mathrm{Sn}----\operatorname{Sn}\left(\alpha_{2}\right)\right]$ \\
\hline 5 & $4-$ & 2199.7 & 2203.1 & 100 & $\mathrm{TBA}_{6} \mathrm{H}\left[(\mathrm{K}) \mathrm{Sn}----\mathrm{Sn}\left(\alpha_{2}\right)\right]$ \\
\hline 6 & $4-$ & 2139.4 & 2143.1 & 24.0 & $\mathrm{TBA}_{5} \mathrm{H}_{2}\left[(\mathrm{~K}) \mathrm{Sn}----\mathrm{Sn}\left(\alpha_{2}\right)\right]$ \\
\hline 7 & $5-$ & 1759.7 & 1762.0 & 46.5 & $\operatorname{TBA}_{6}\left[(\mathrm{~K}) \mathrm{Sn}----\operatorname{Sn}\left(\alpha_{2}\right)\right]$ \\
\hline 8 & $5-$ & 1711.3 & 1712.8 & 68.5 & $\mathrm{TBA}_{5} \mathrm{H}\left[(\mathrm{K}) \mathrm{Sn}----\mathrm{Sn}\left(\alpha_{2}\right)\right]$ \\
\hline 9 & $5-$ & 1663.0 & 1663.0 & 20.9 & $\mathrm{TBA}_{4} \mathrm{H}_{2}\left[(\mathrm{~K}) \mathrm{Sn}----\mathrm{Sn}\left(\alpha_{2}\right)\right]$ \\
\hline 10 & $6-$ & 1425.9 & 1427.0 & 25.0 & $\mathrm{TBA}_{5}\left[(\mathrm{~K}) \mathrm{Sn}----\mathrm{Sn}\left(\alpha_{2}\right)\right]$ \\
\hline 11 & $6-$ & 1385.7 & 1386.8 & 30.9 & $\mathrm{TBA}_{4} \mathrm{H}\left[(\mathrm{K}) \mathrm{Sn}----\mathrm{Sn}\left(\alpha_{2}\right)\right]$ \\
\hline 12 & $6-$ & 1345.4 & 1346.0 & 8.9 & $\mathrm{TBA}_{3} \mathrm{H}_{2}\left[(\mathrm{~K}) \mathrm{Sn}----\mathrm{Sn}\left(\alpha_{2}\right)\right]$ \\
\hline 13 & $7-$ & 1187.6 & 1189.0 & 25.8 & $\mathrm{TBA}_{4}\left[(\mathrm{~K}) \mathrm{Sn}----\operatorname{Sn}\left(\alpha_{2}\right)\right]$ \\
\hline 14 & 7- & 1153.1 & 1154.5 & 29.6 & $\mathrm{TBA}_{3} \mathrm{H}\left[(\mathrm{K}) \mathrm{Sn}----\mathrm{Sn}\left(\alpha_{2}\right)\right]$ \\
\hline 16 & $8-$ & 948.5 & 949.1 & 23.4 & $\mathrm{TBAH}_{2}\left[(\mathrm{~K}) \mathrm{Sn}----\operatorname{Sn}\left(\alpha_{2}\right)\right]$ \\
\hline
\end{tabular}




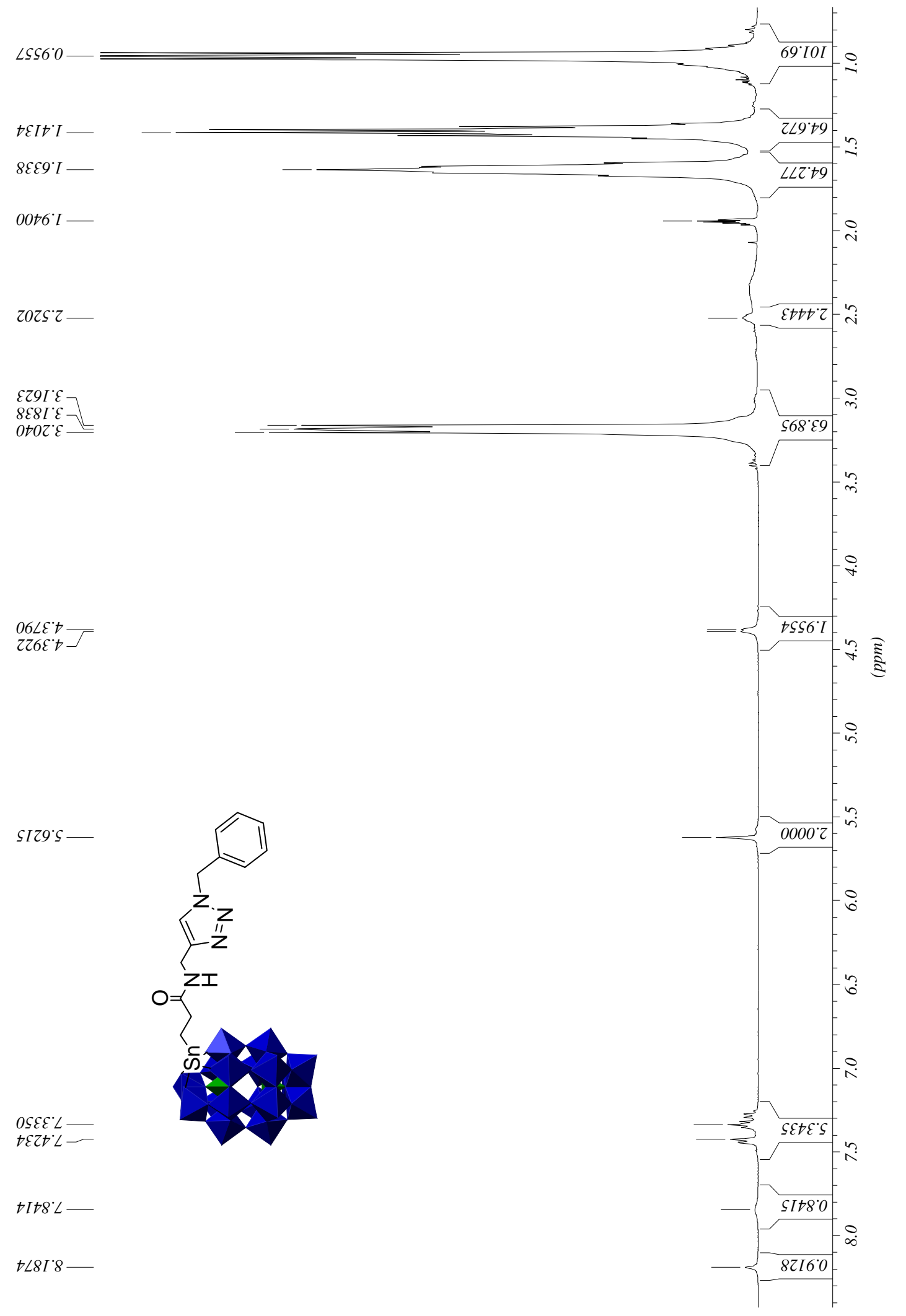




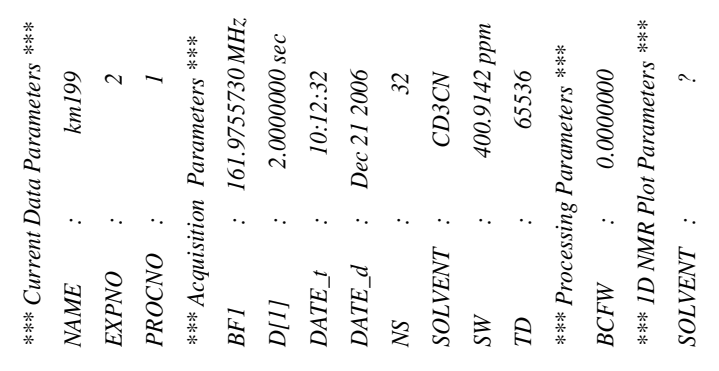

$\frac{\infty}{\infty}$

$\left.\begin{array}{l}\operatorname{sos} 6^{-} 6^{-} \\ 6 t t t^{\circ} 6^{-} \\ \varepsilon 6 s \varepsilon^{-} 6^{-}\end{array}\right]$

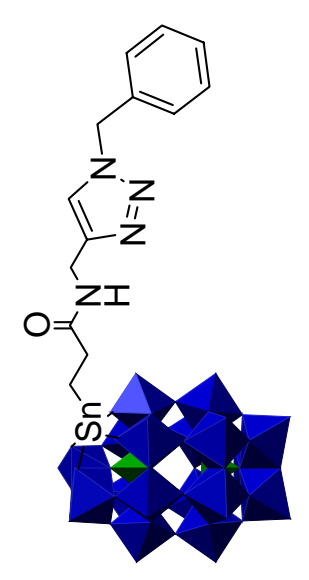




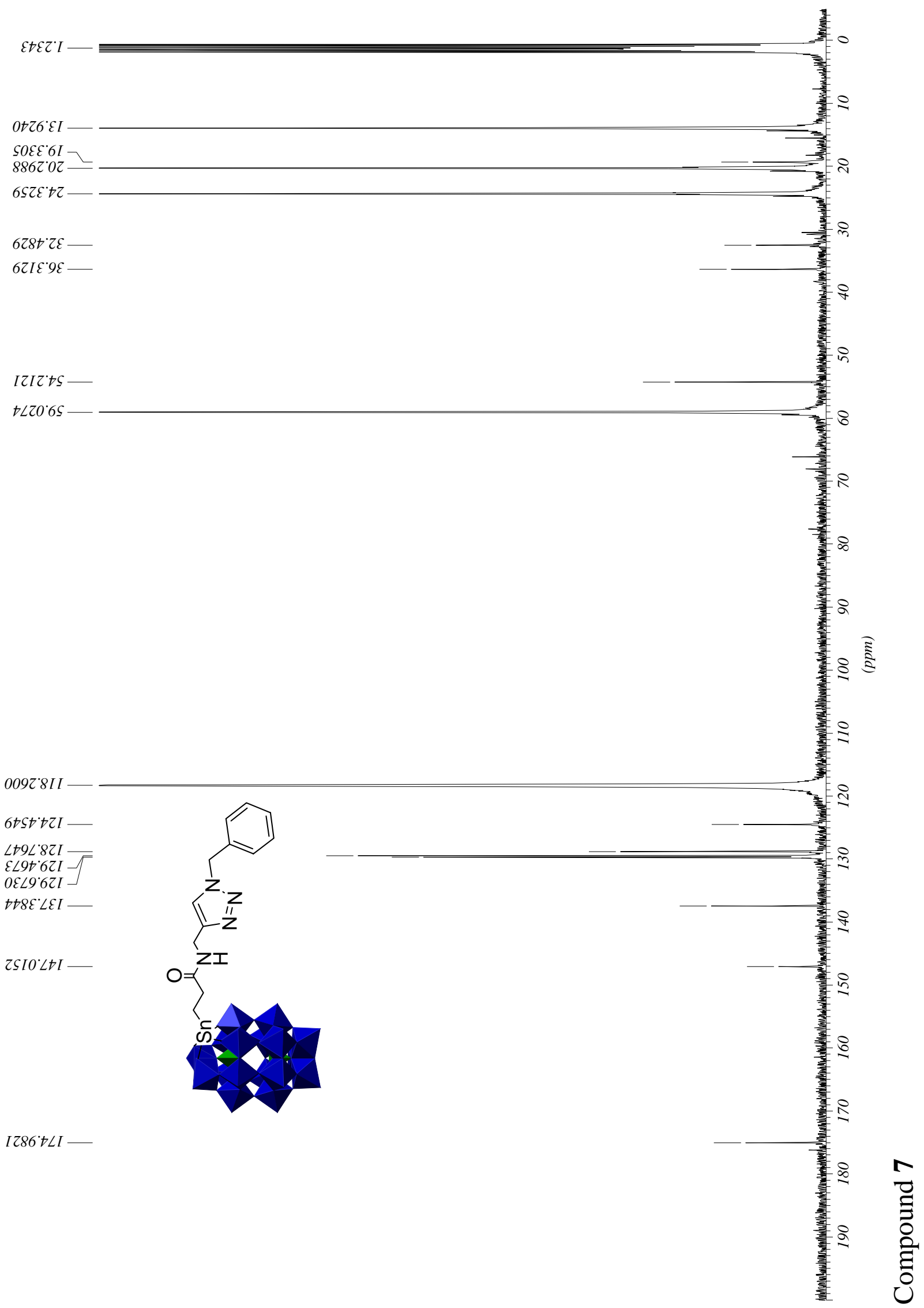




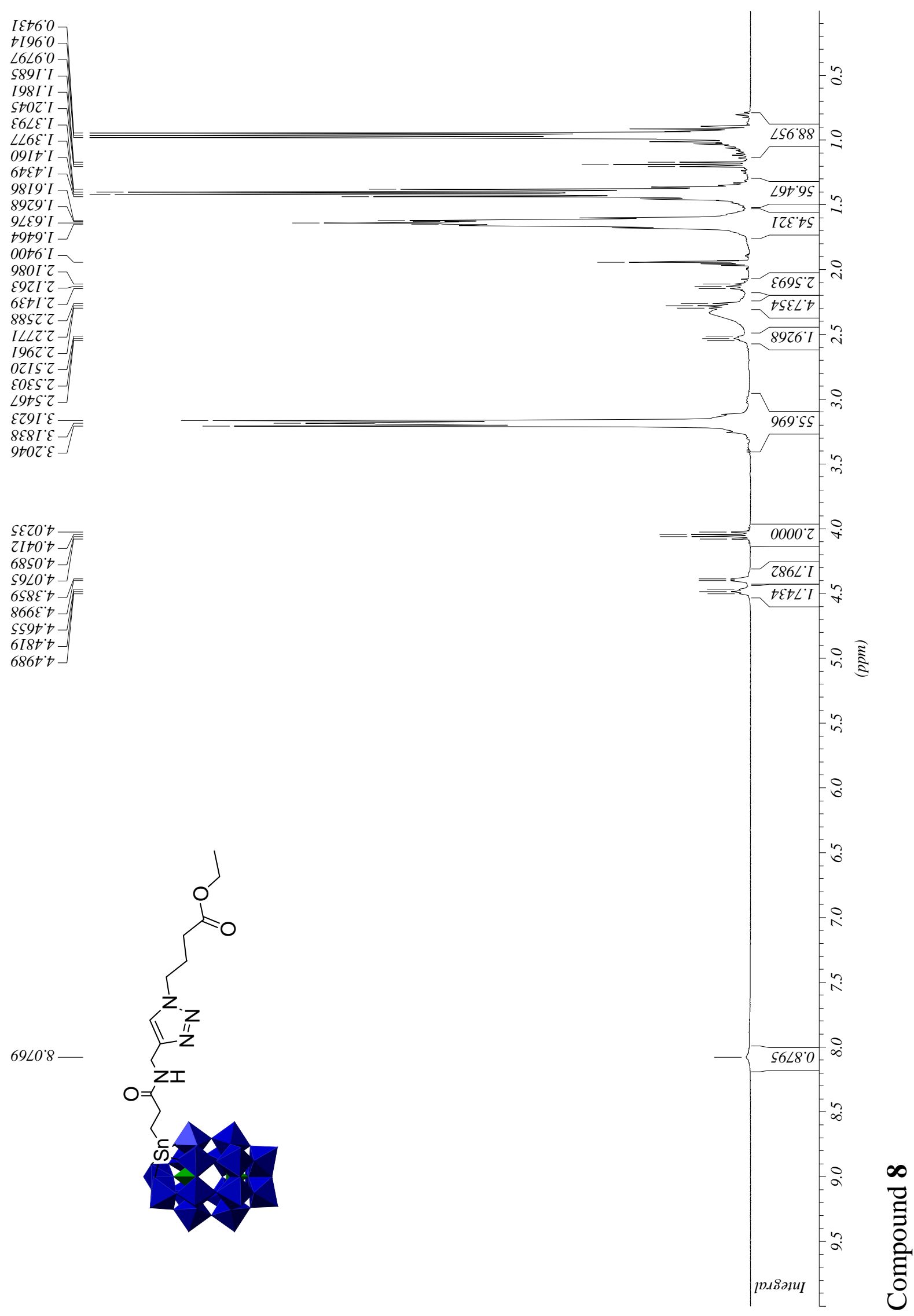




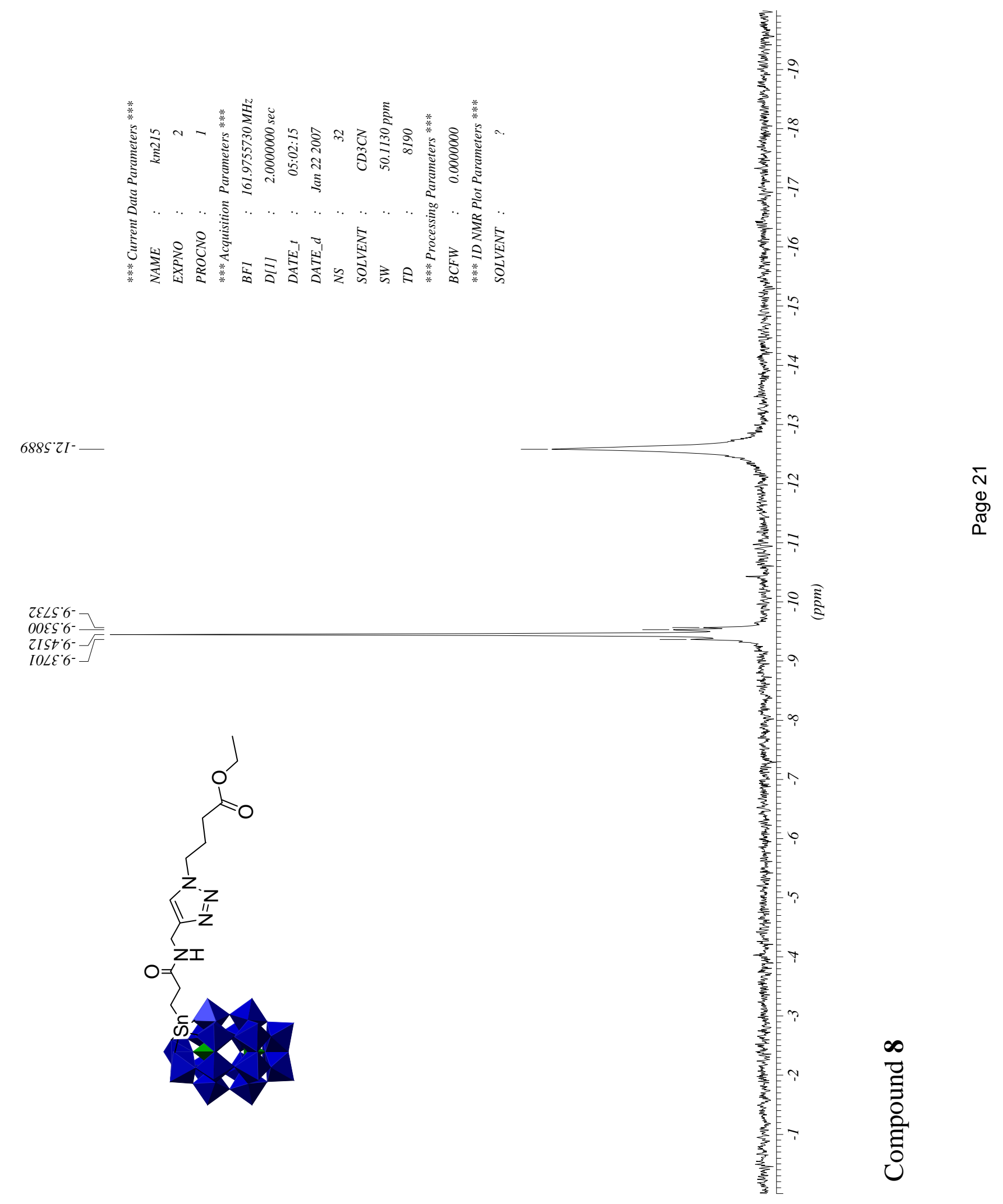




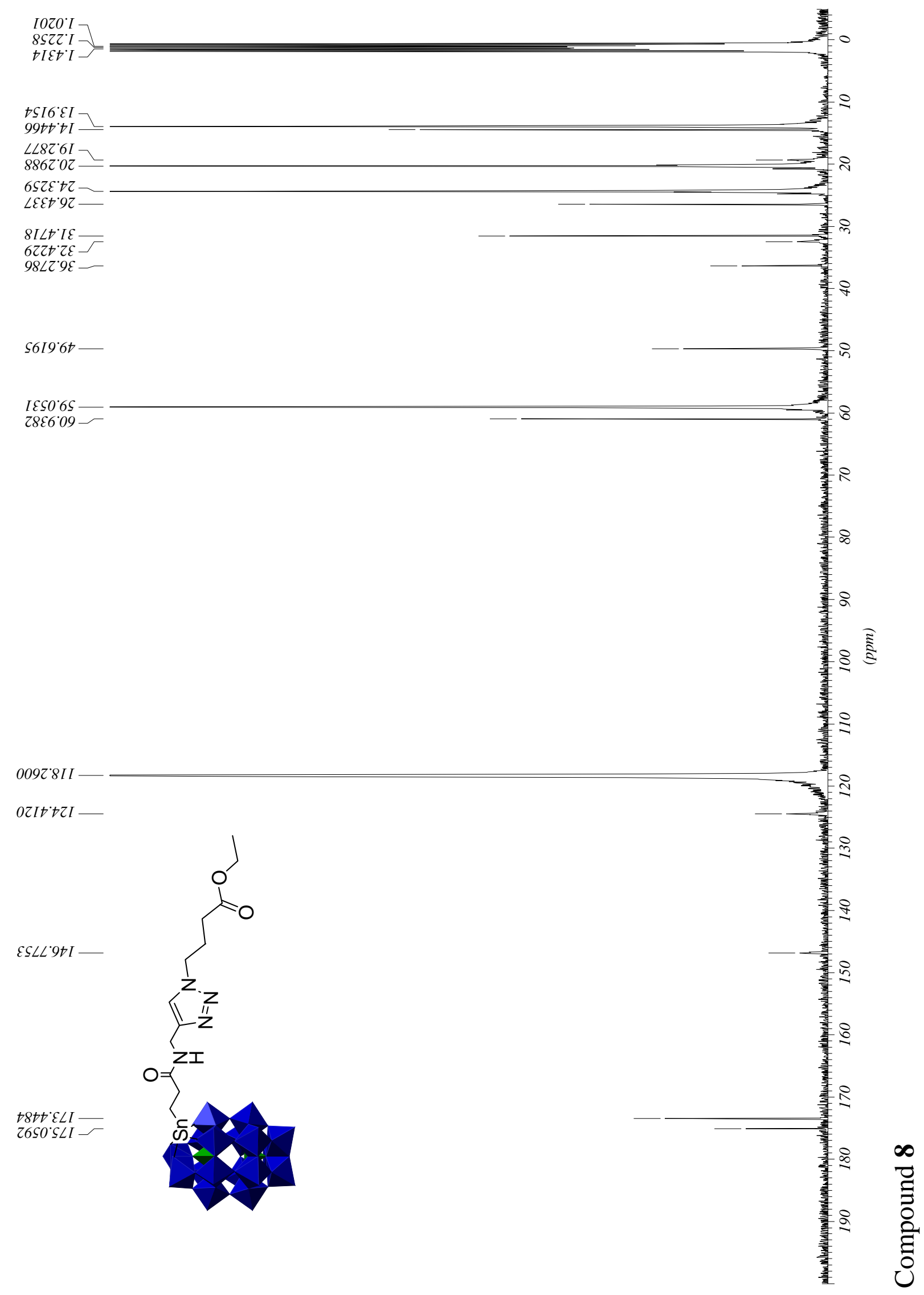




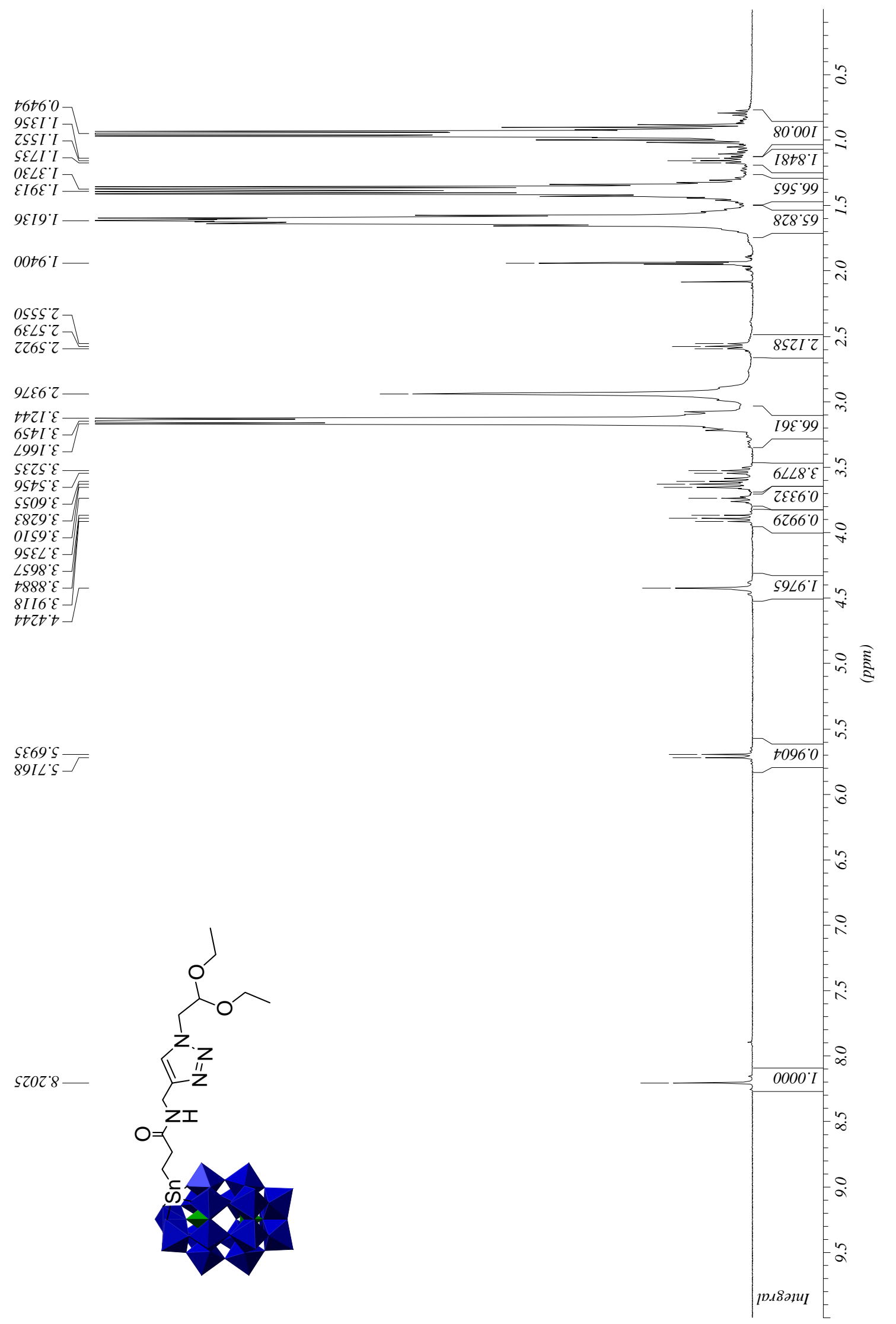



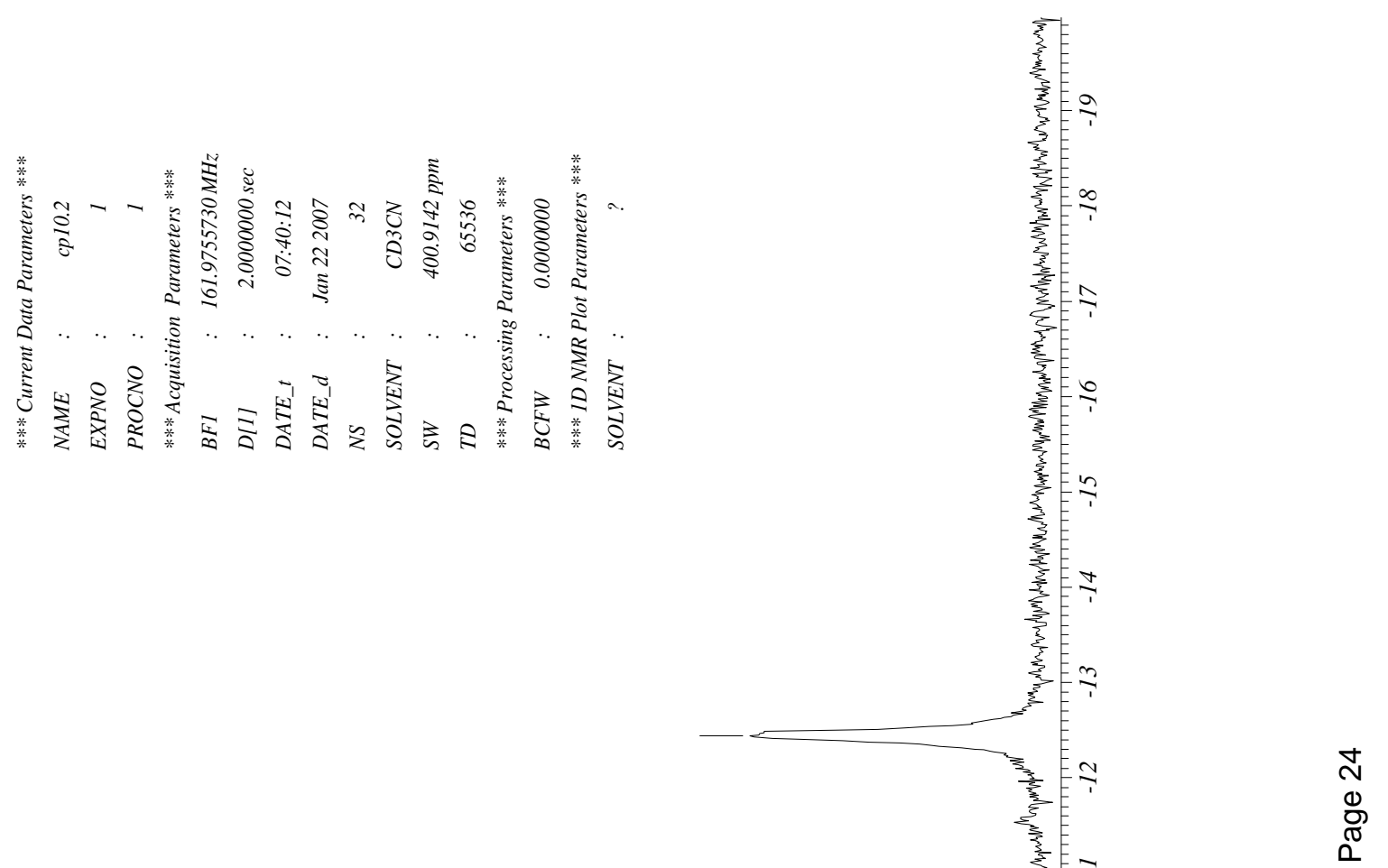

$S 6 \angle S^{\circ} 6^{-}$

옹

$8 \varepsilon 60^{\circ} 6^{-}-$

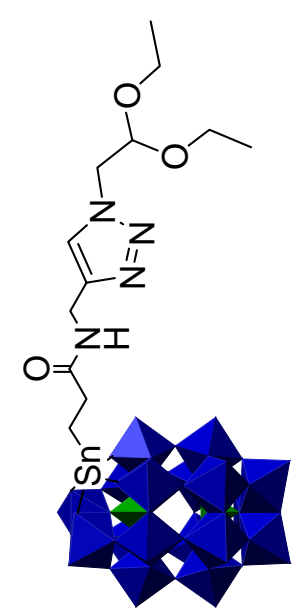

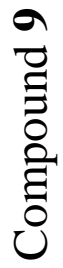




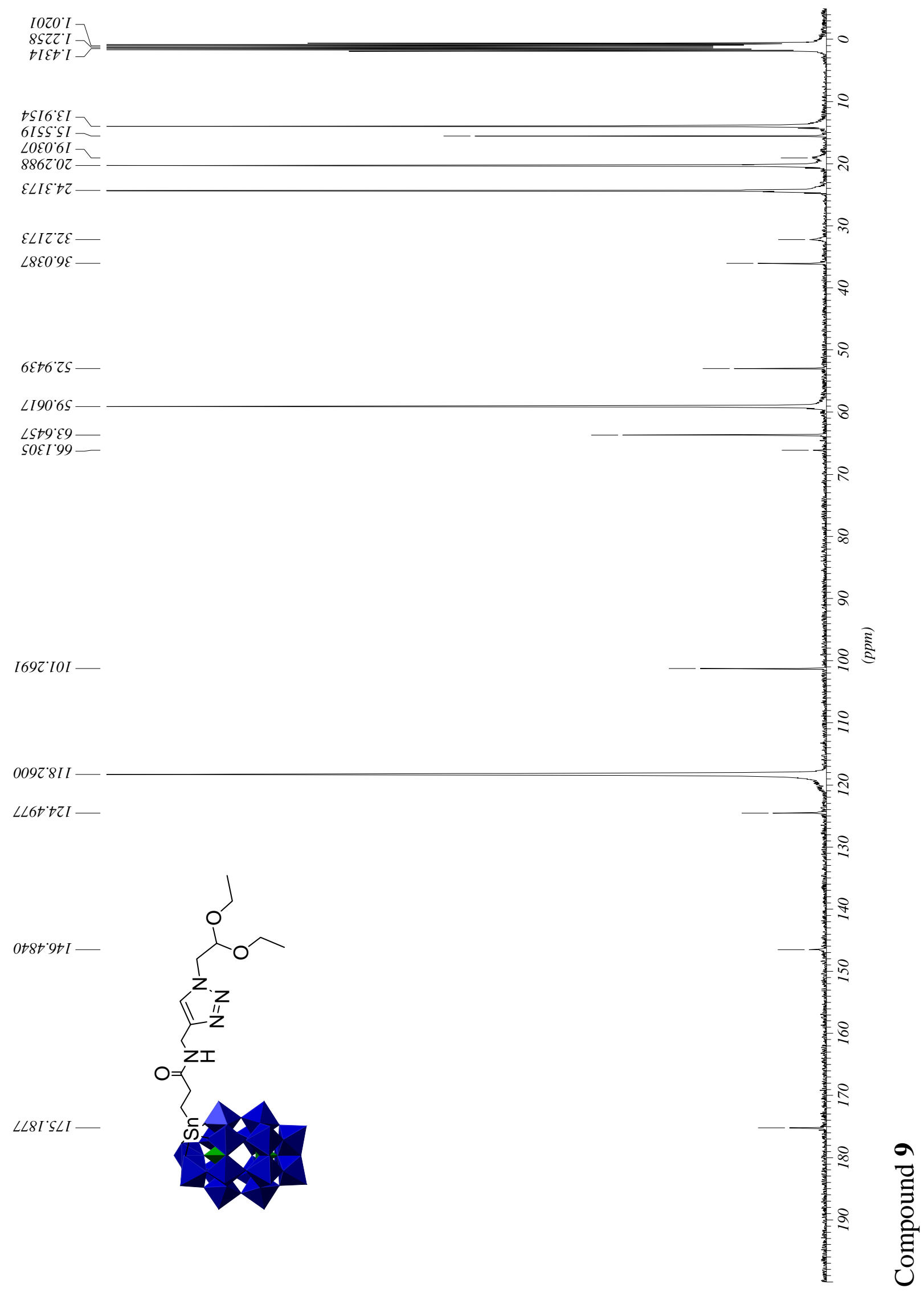




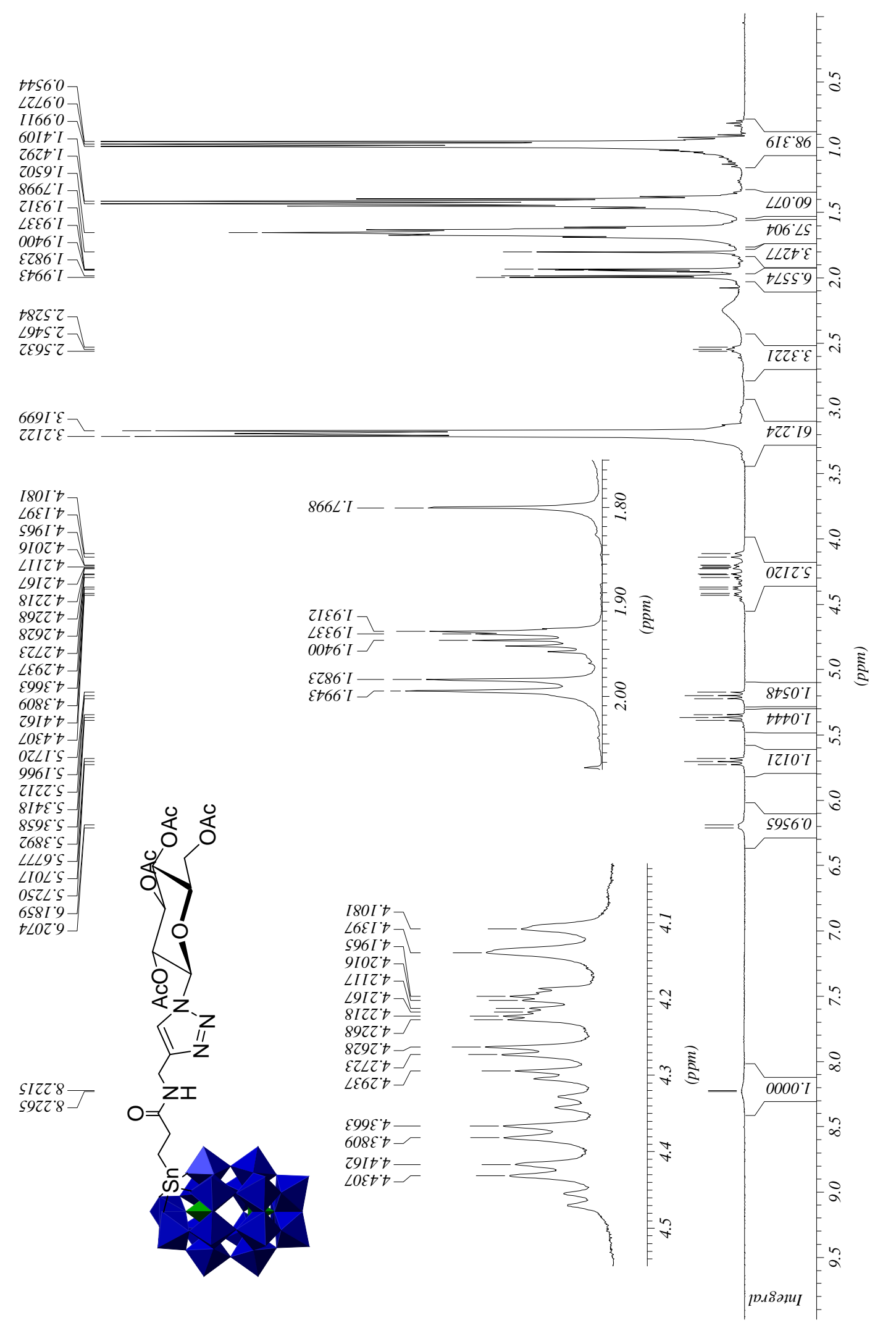




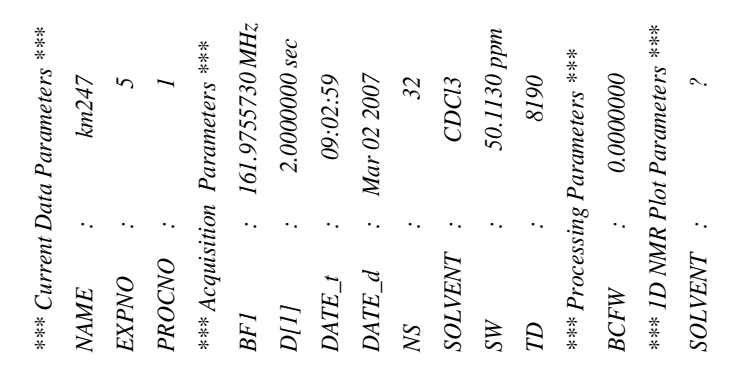

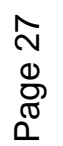

$\angle I t t^{\circ} Z I^{-}$

6ESt' 6 -

$+\angle L E 6^{-}$
ZOOE $6^{-}$

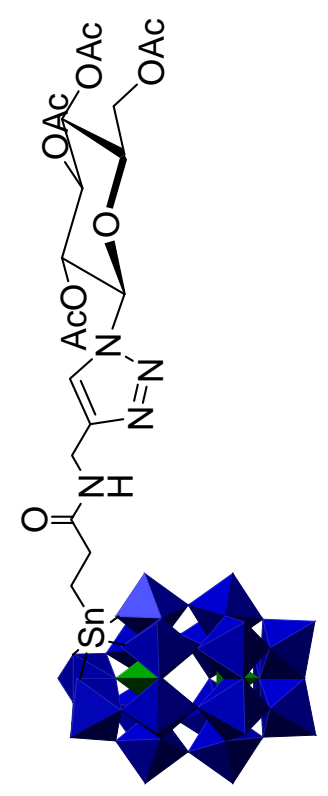

0
$\overline{0}$
0
0
0 


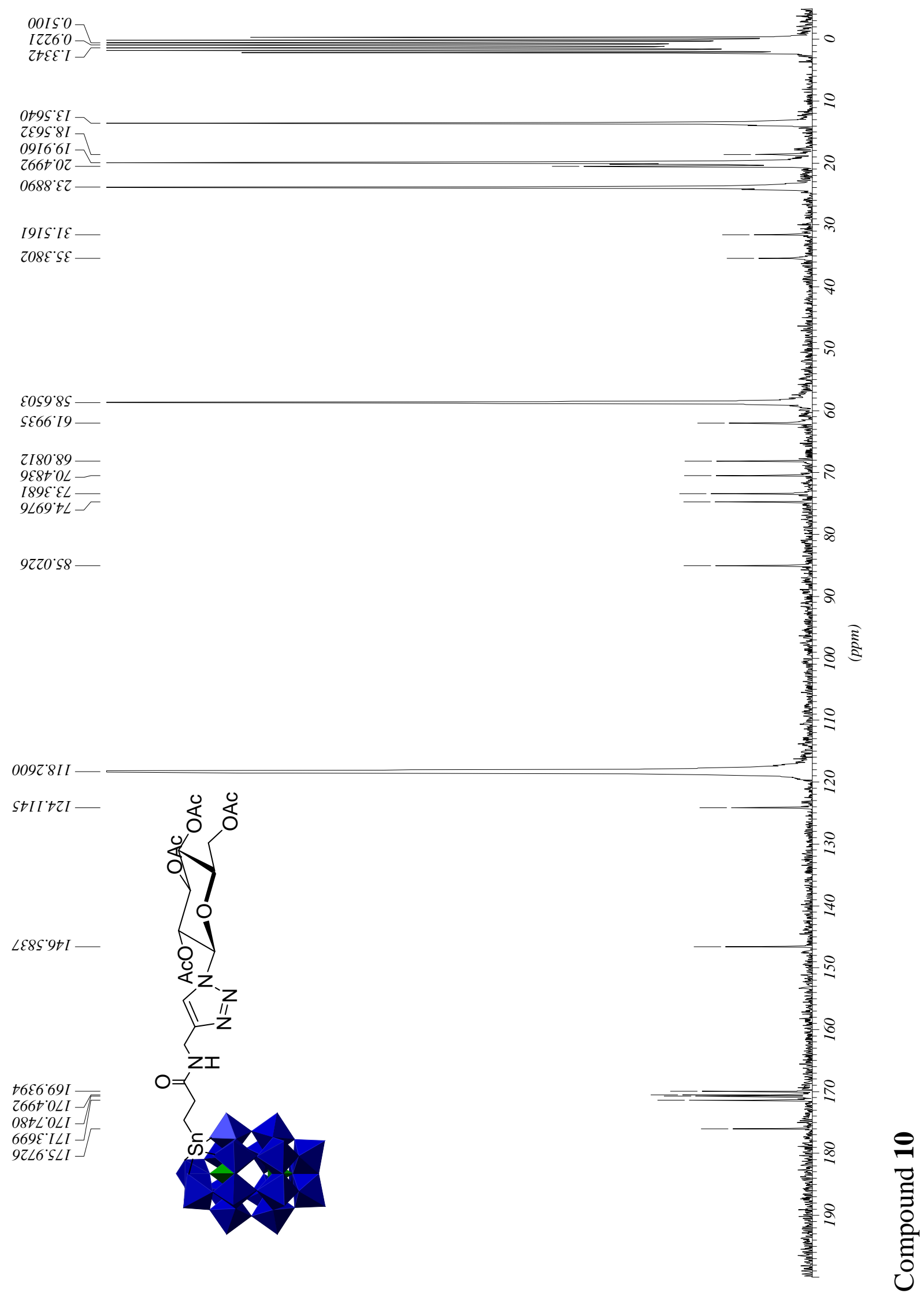




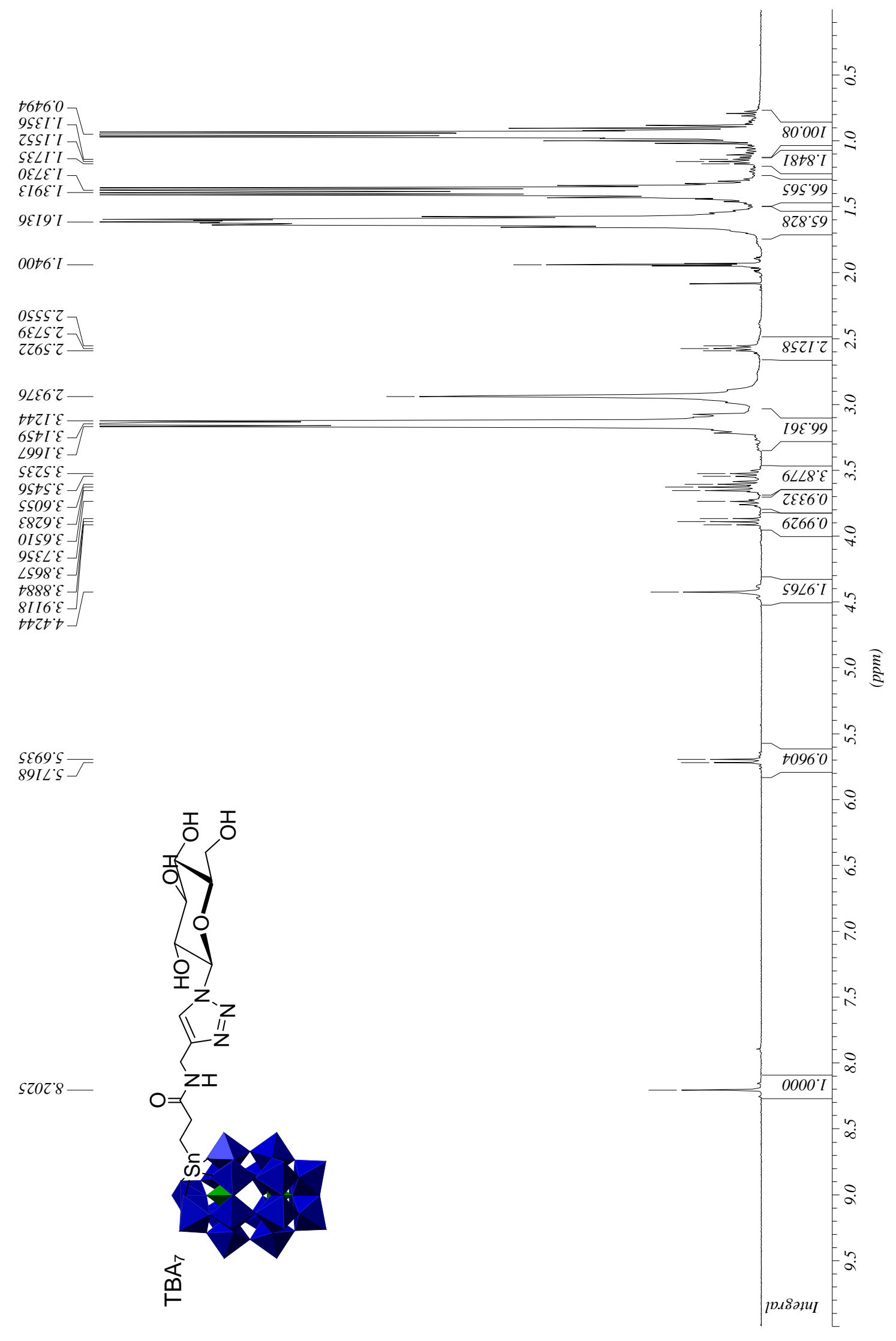



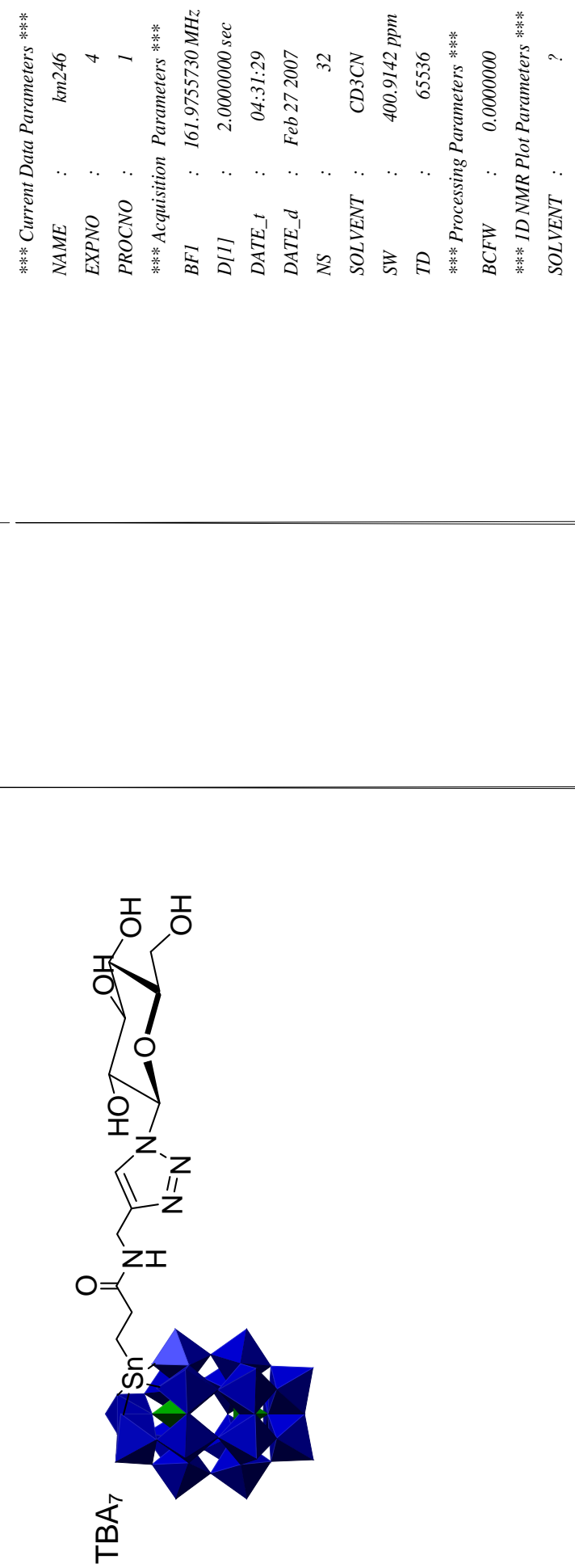


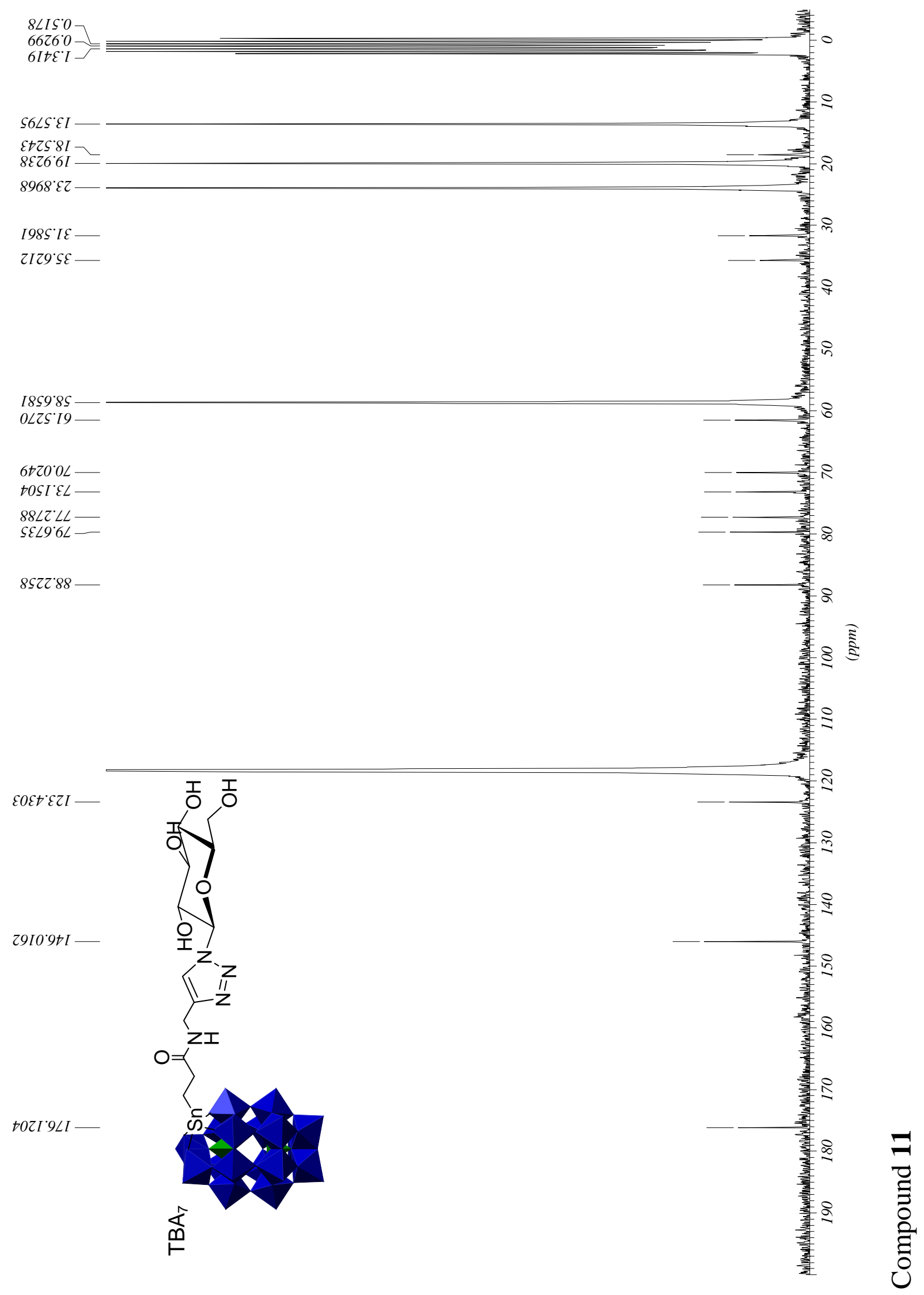




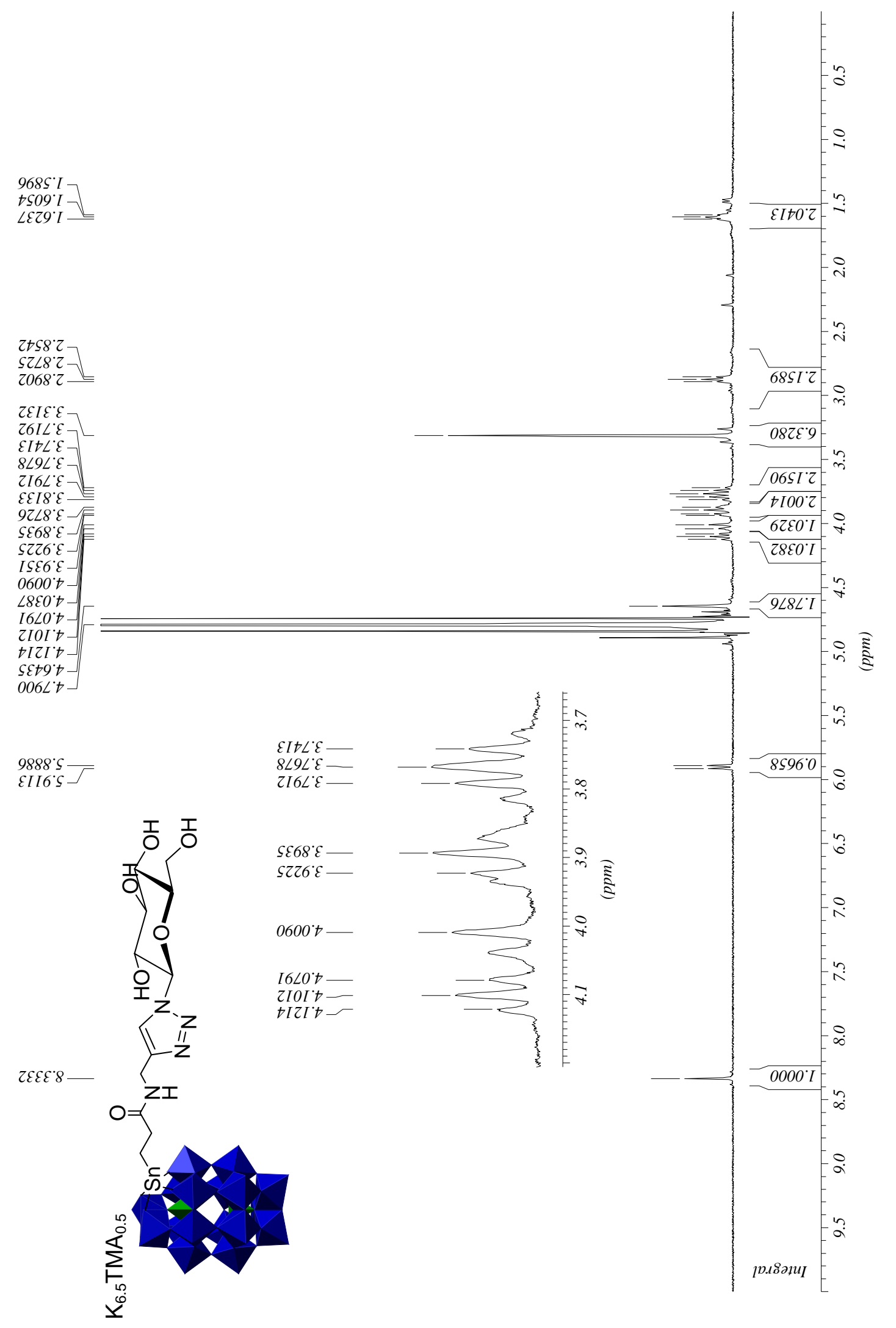



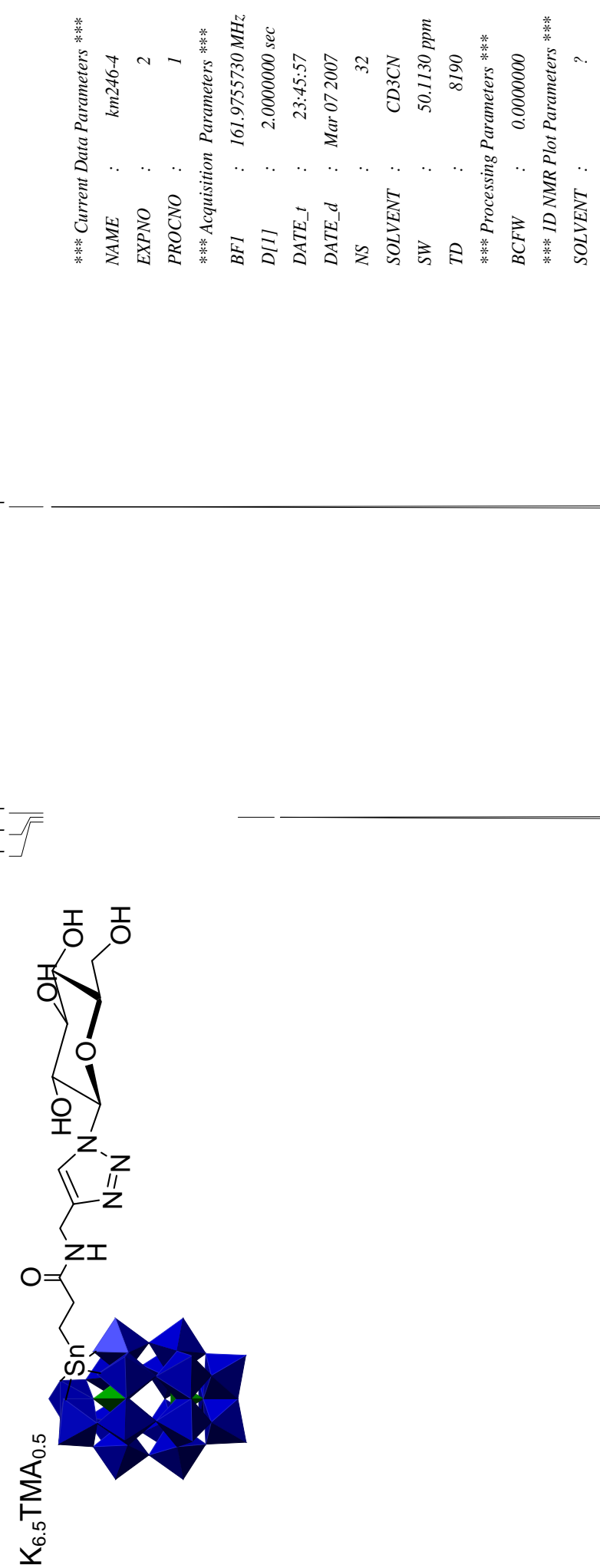


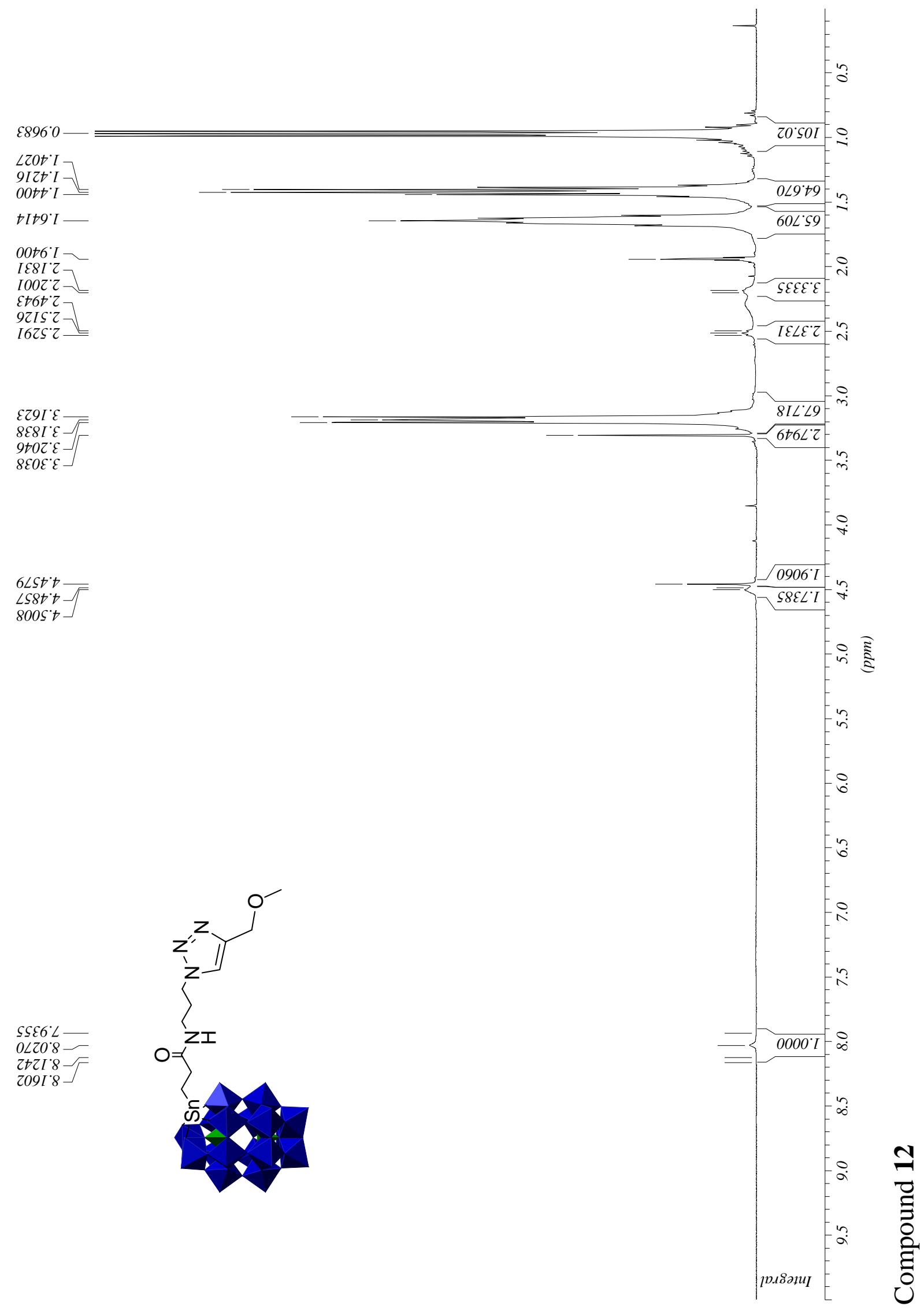




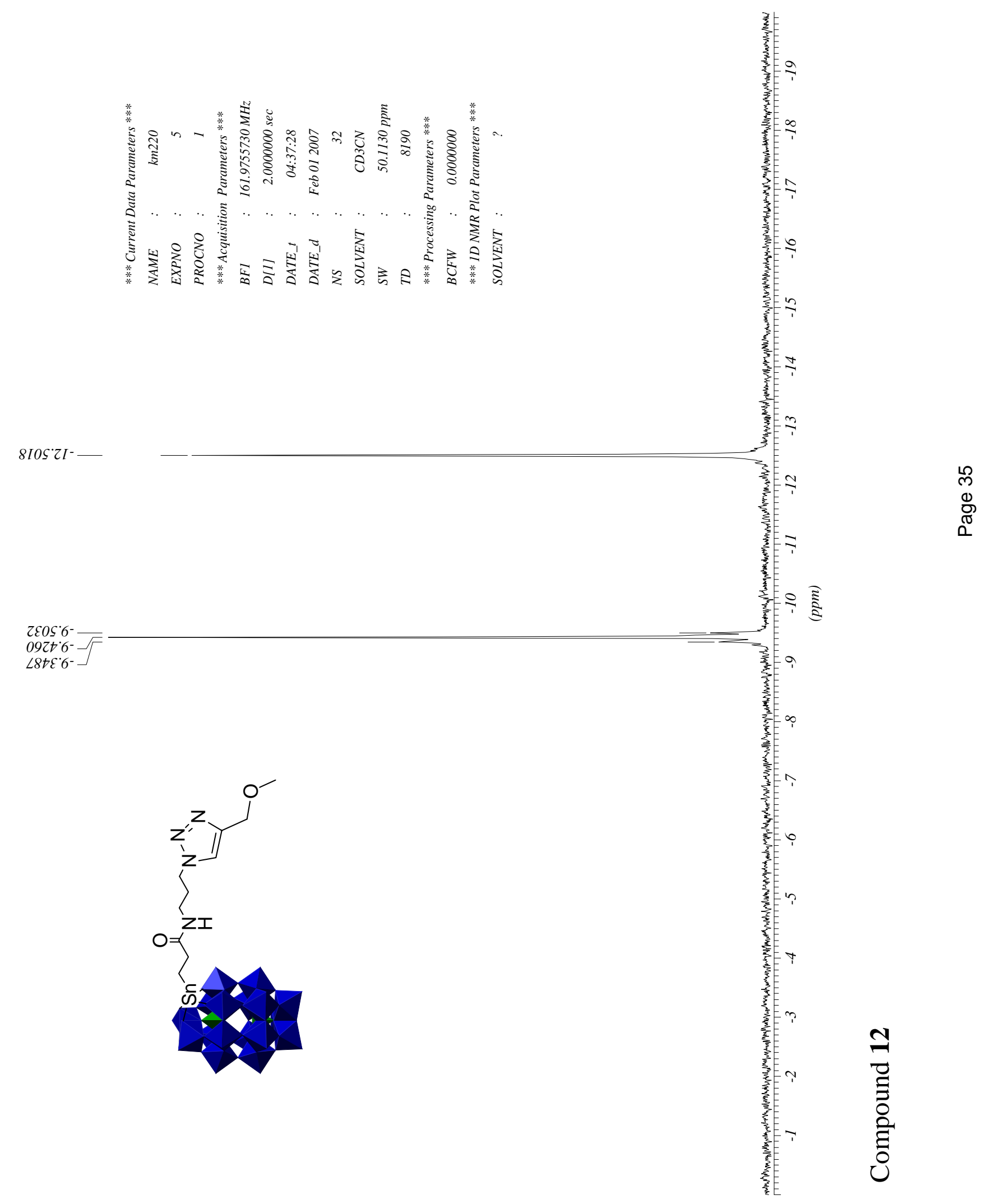




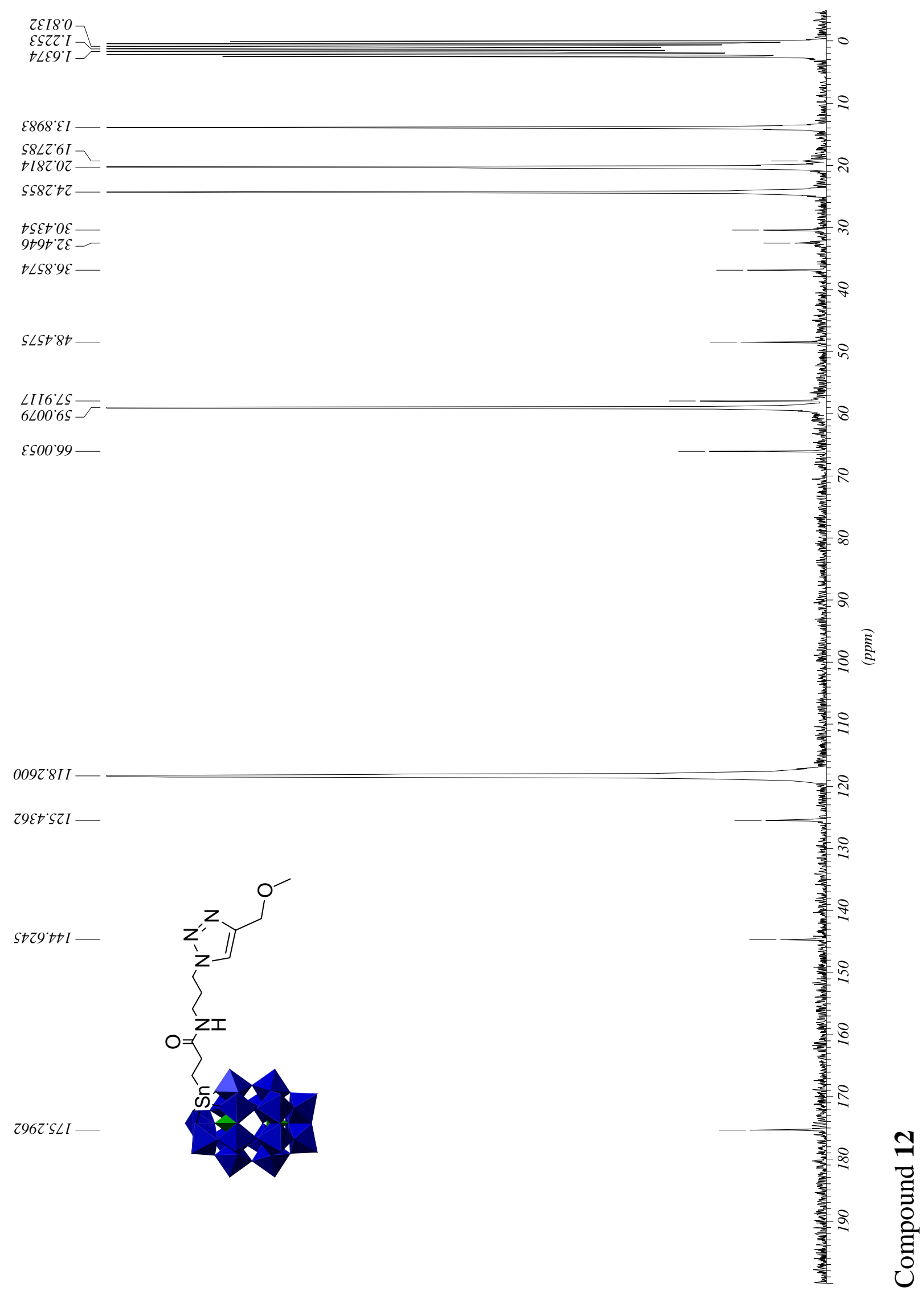




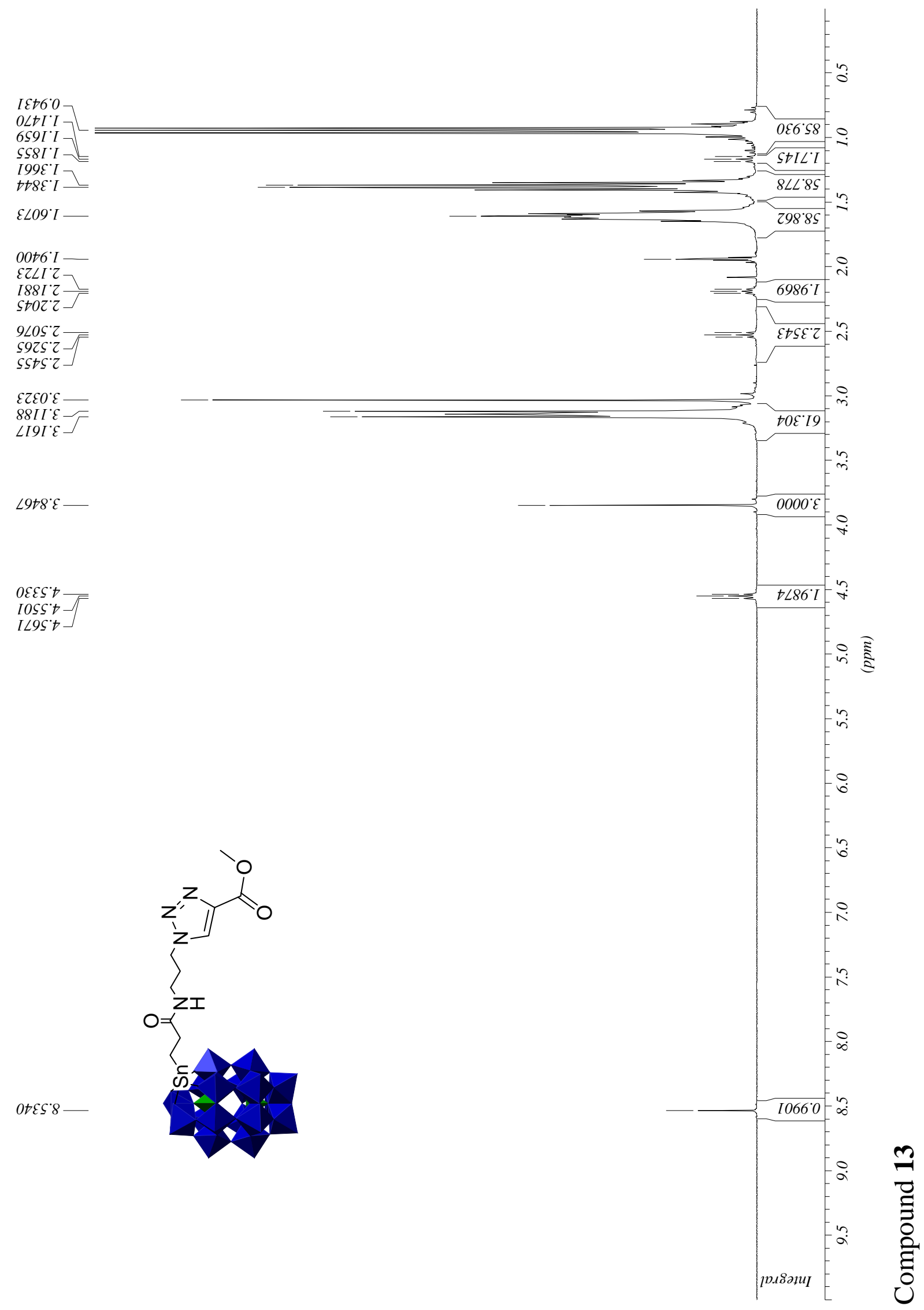



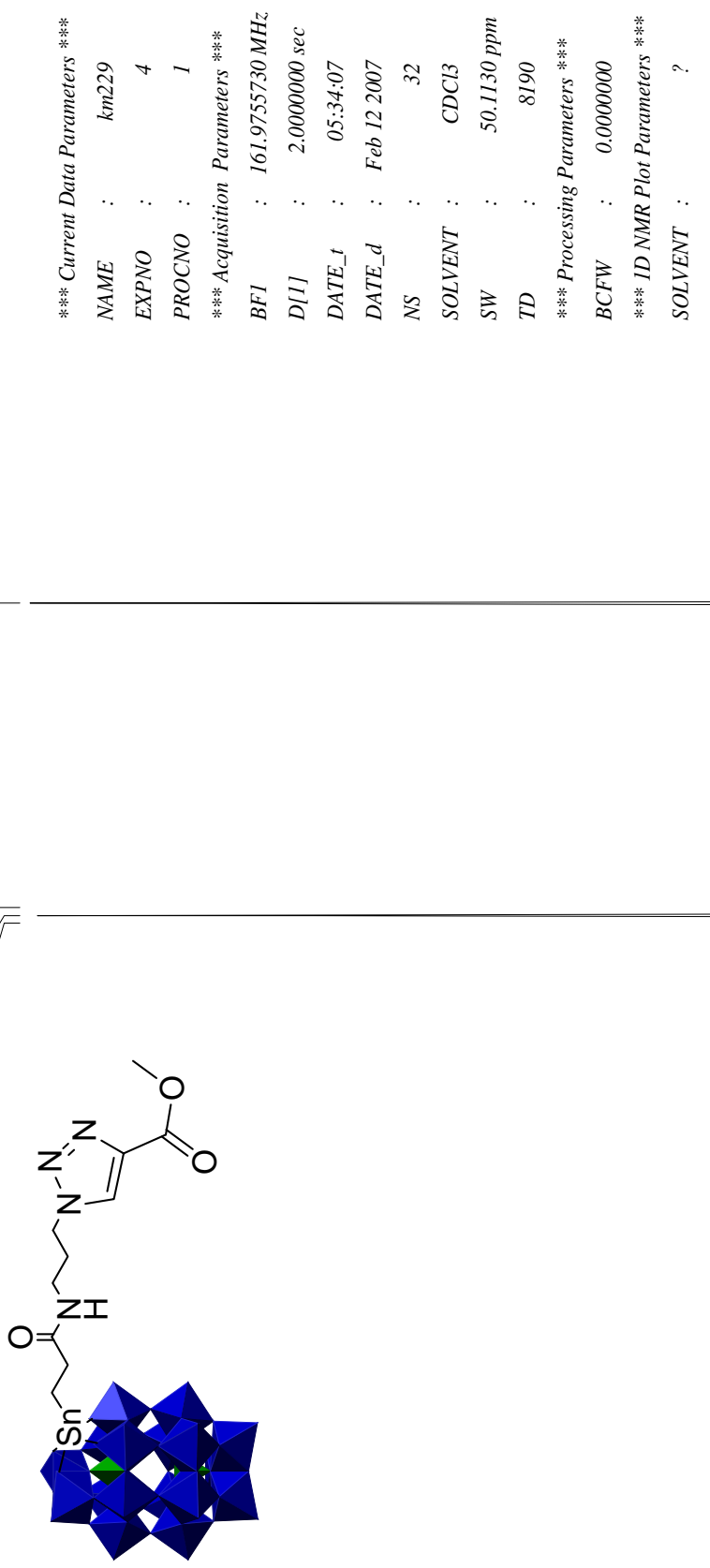


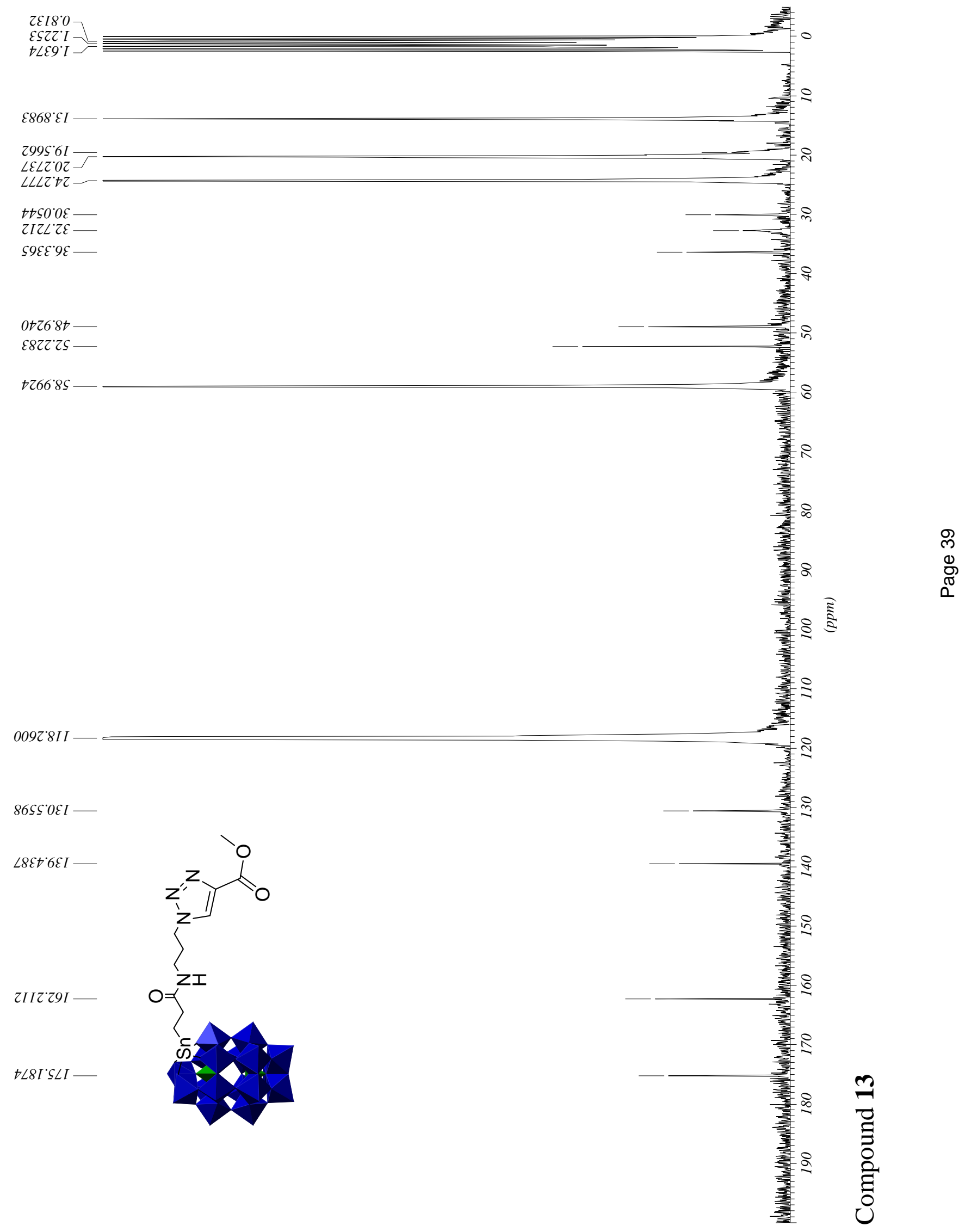




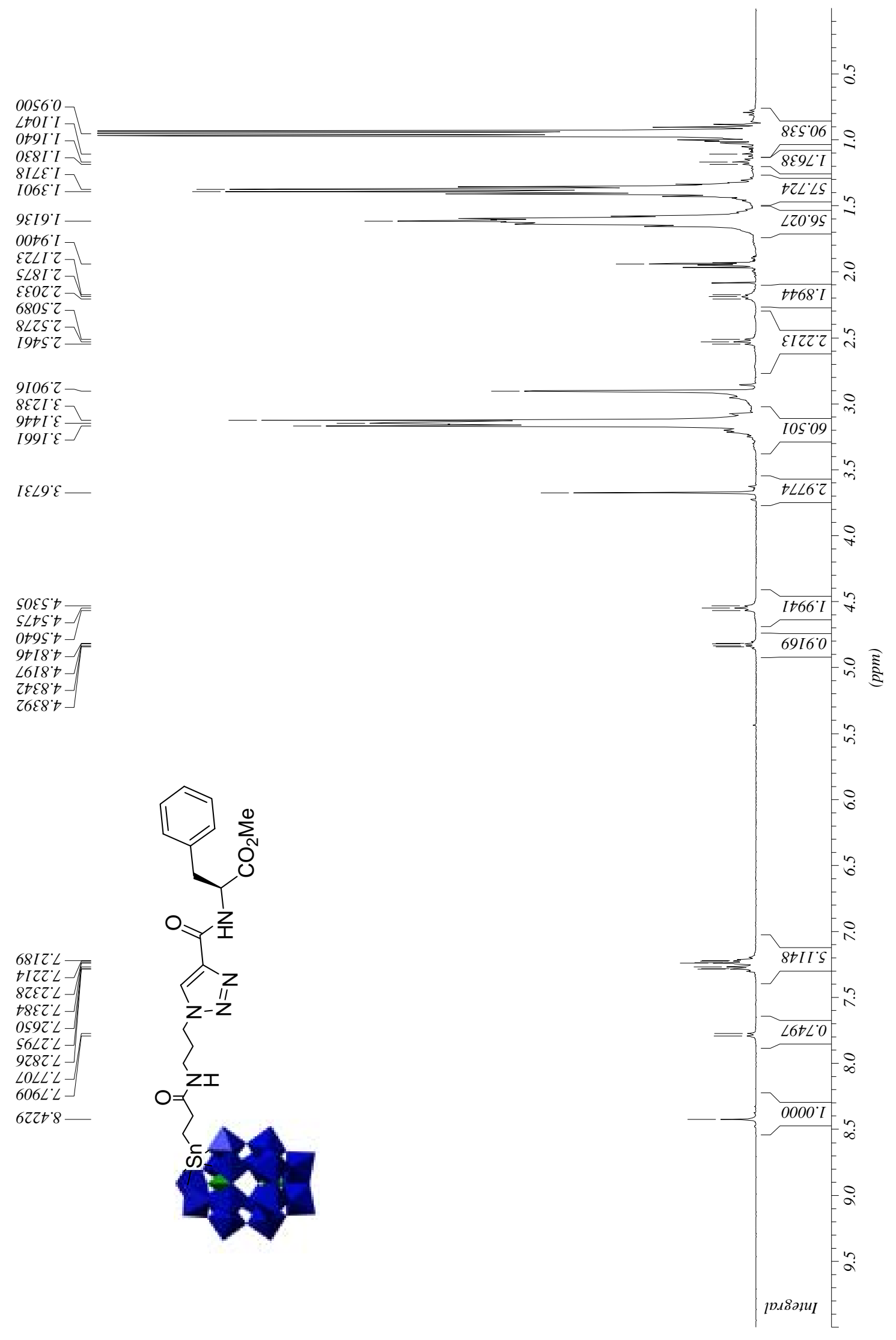




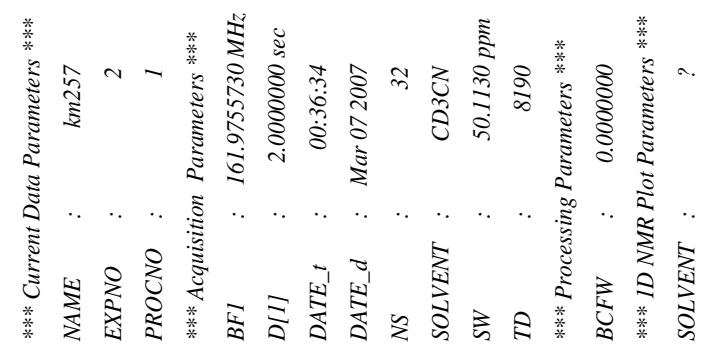

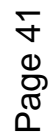

$\angle L E S^{\circ} Z I^{-}$

9EEt $6^{-}$

9SSE $6^{-}$

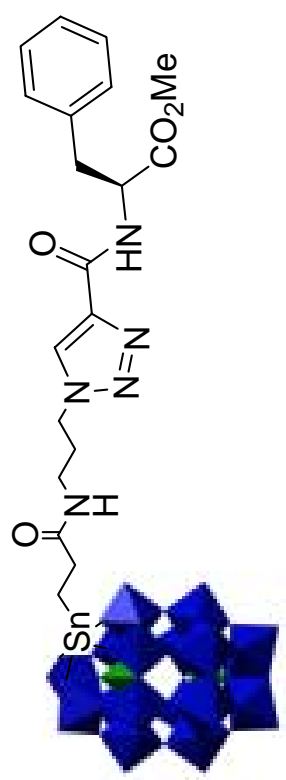




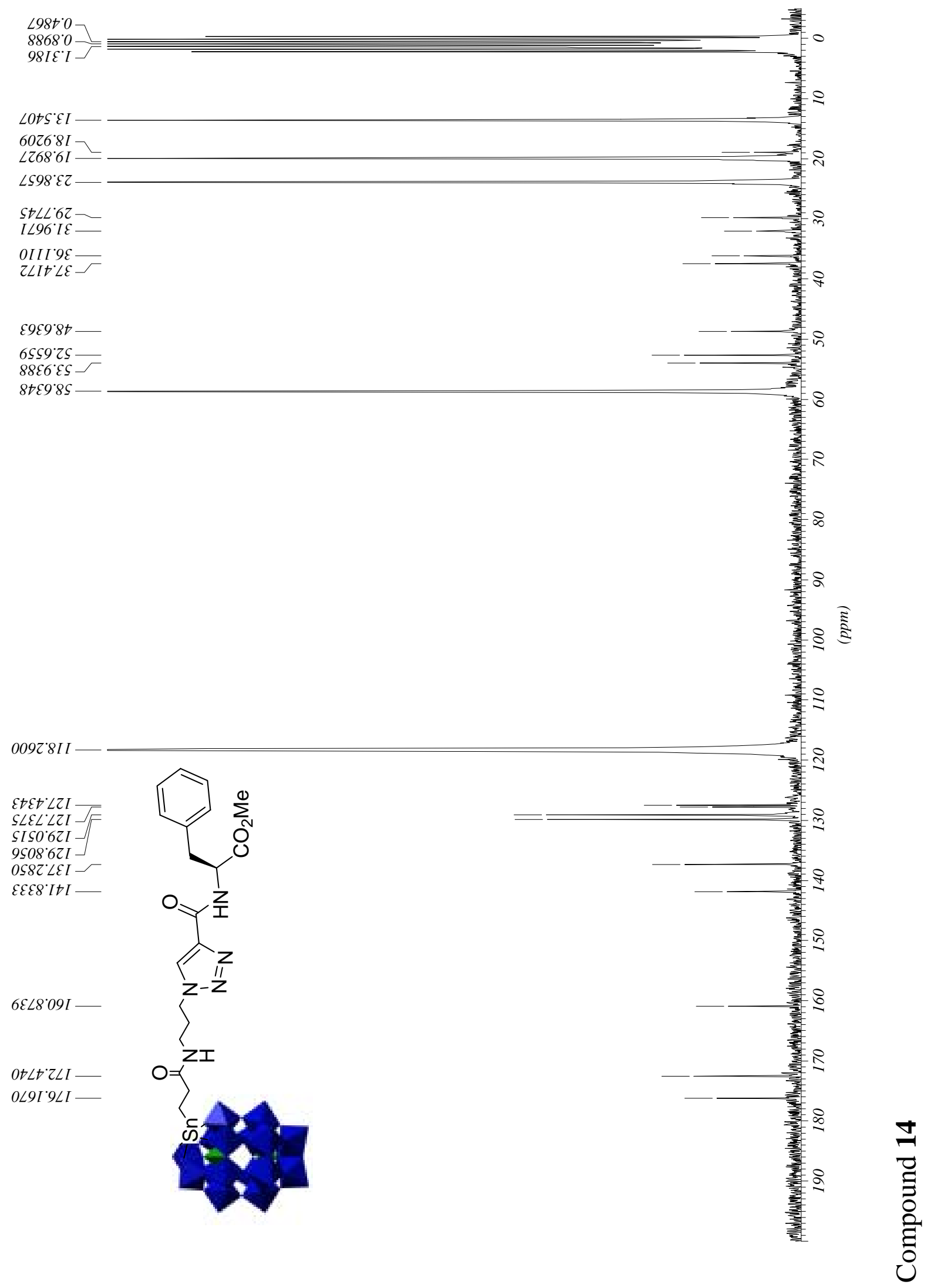




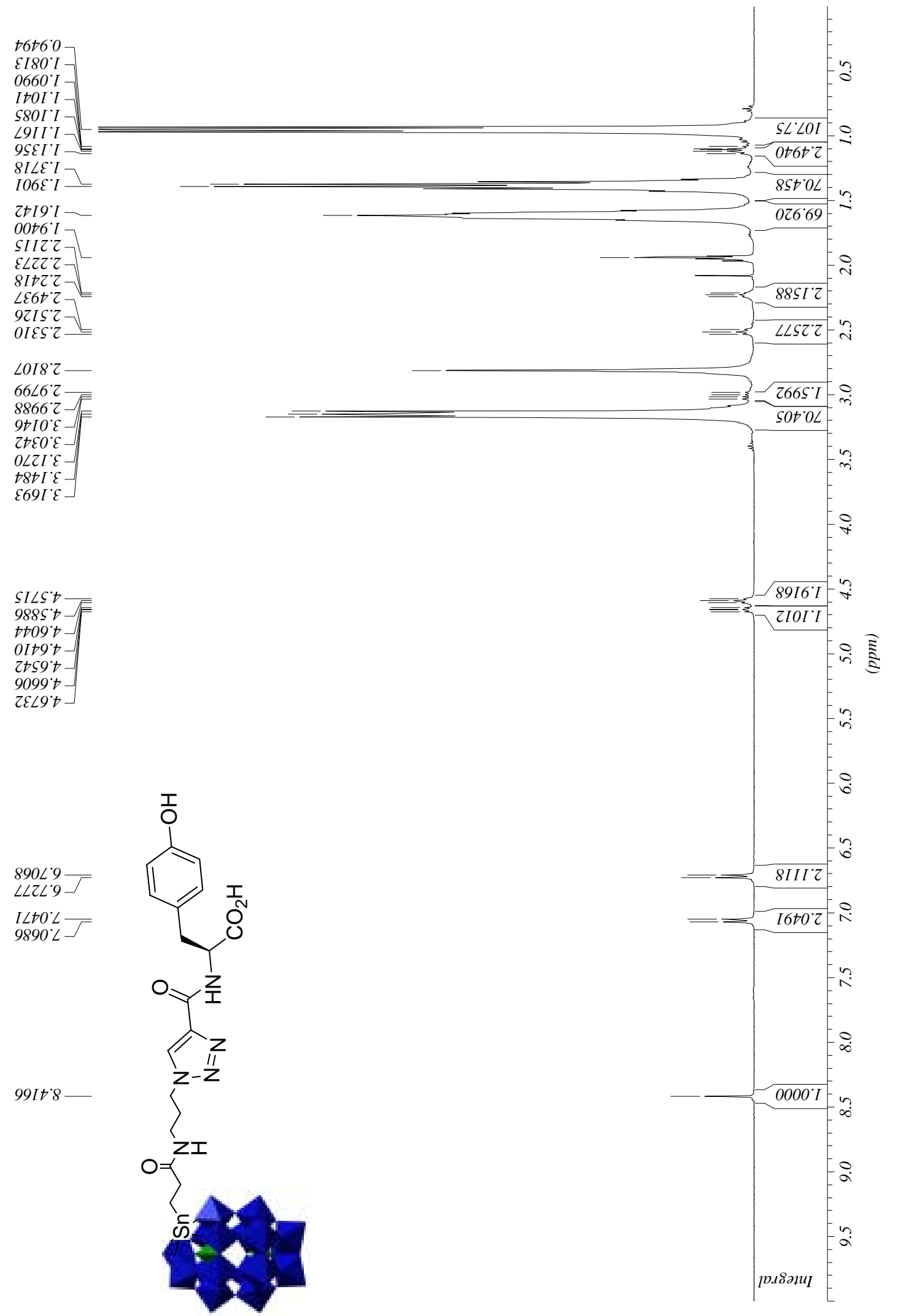




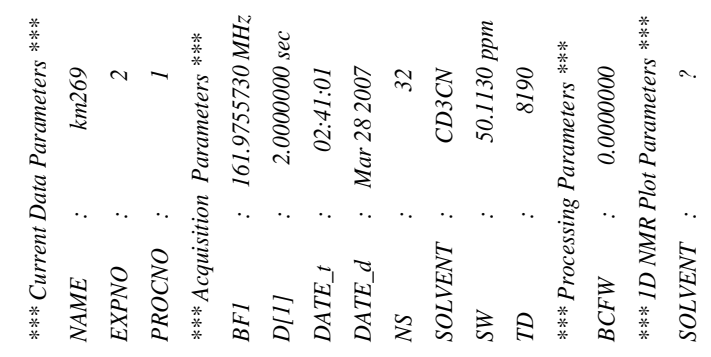

ํㅜㄴ

0

I689' ZI- $^{-}$

EOLt' $6^{-}-$
$968 \mathcal{C}^{\circ} 6^{-}-$

$\angle 60 \mathcal{E}^{\circ} 6^{-}$

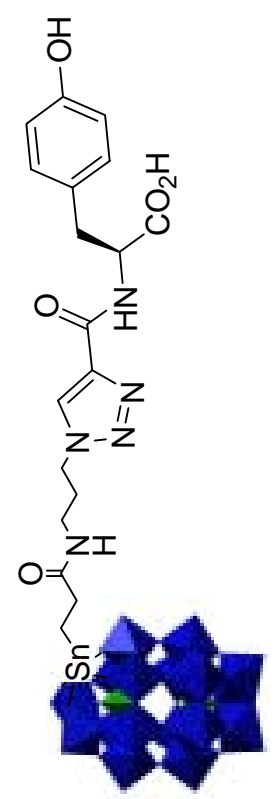




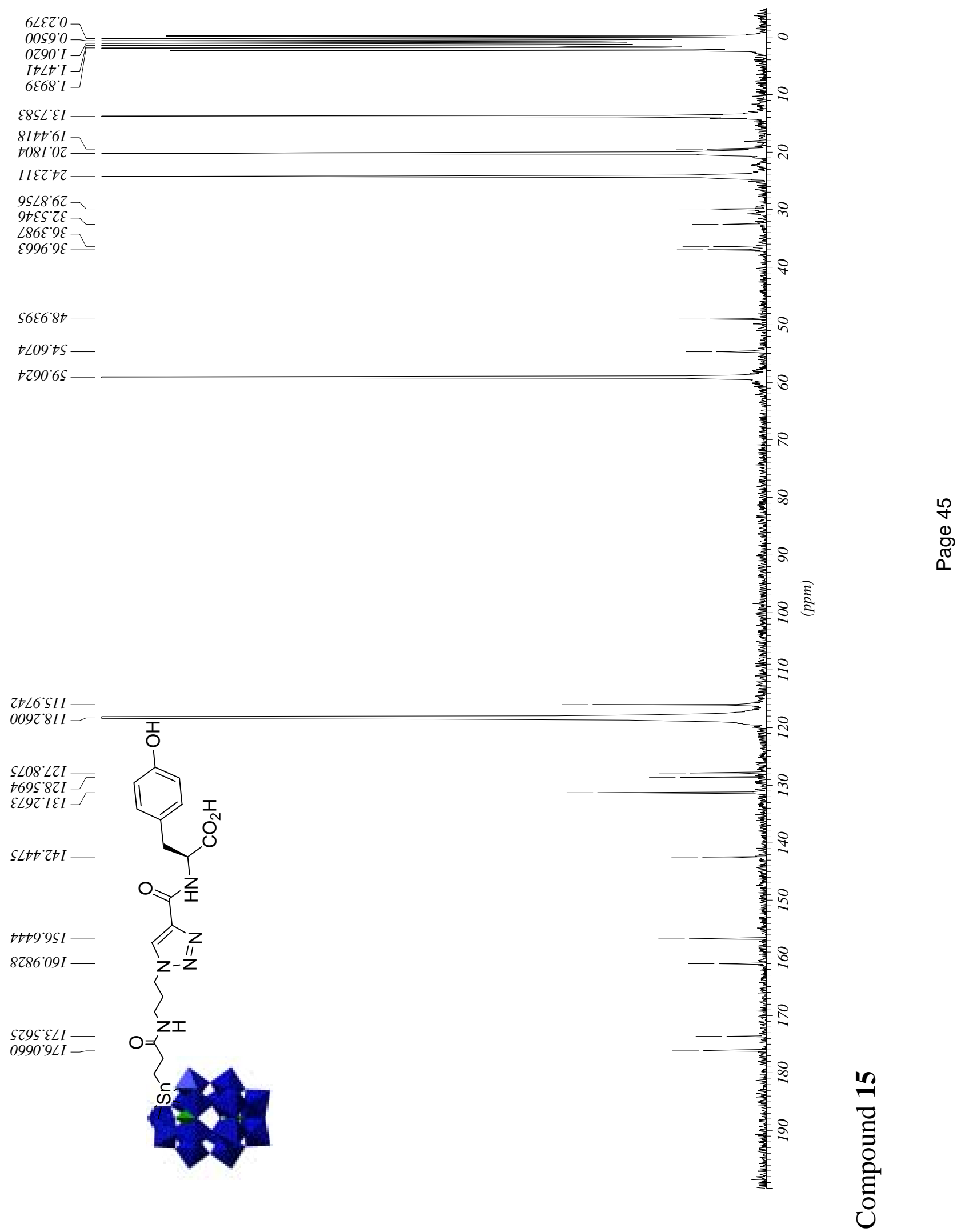




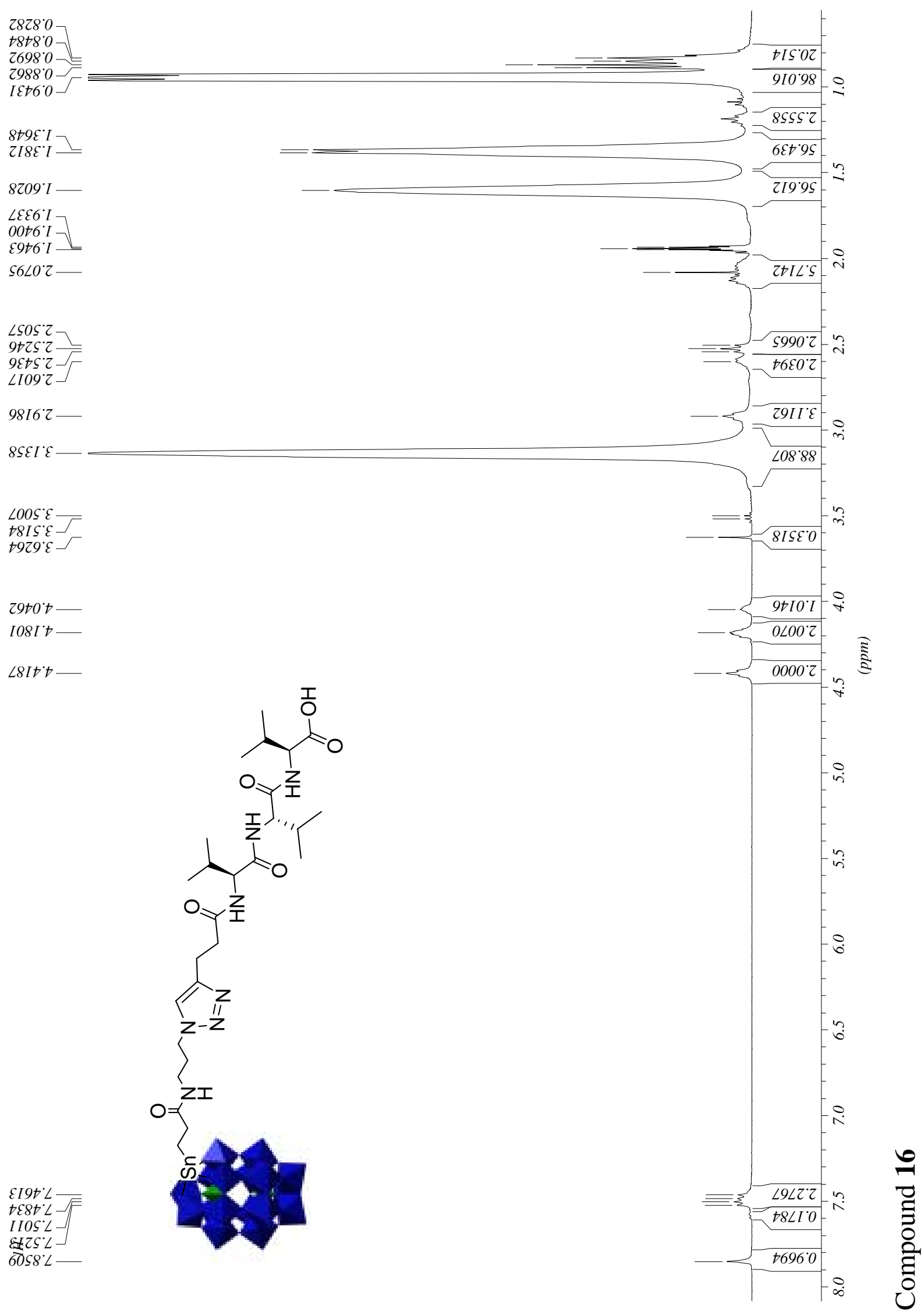



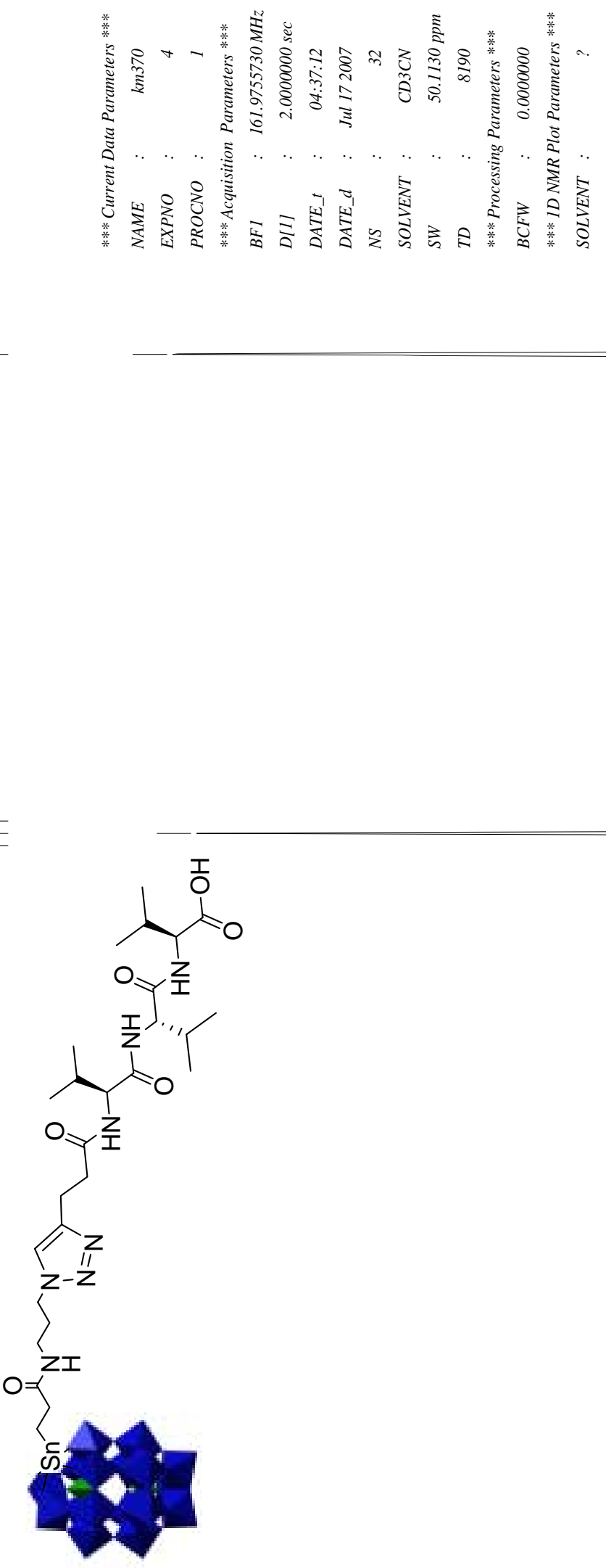


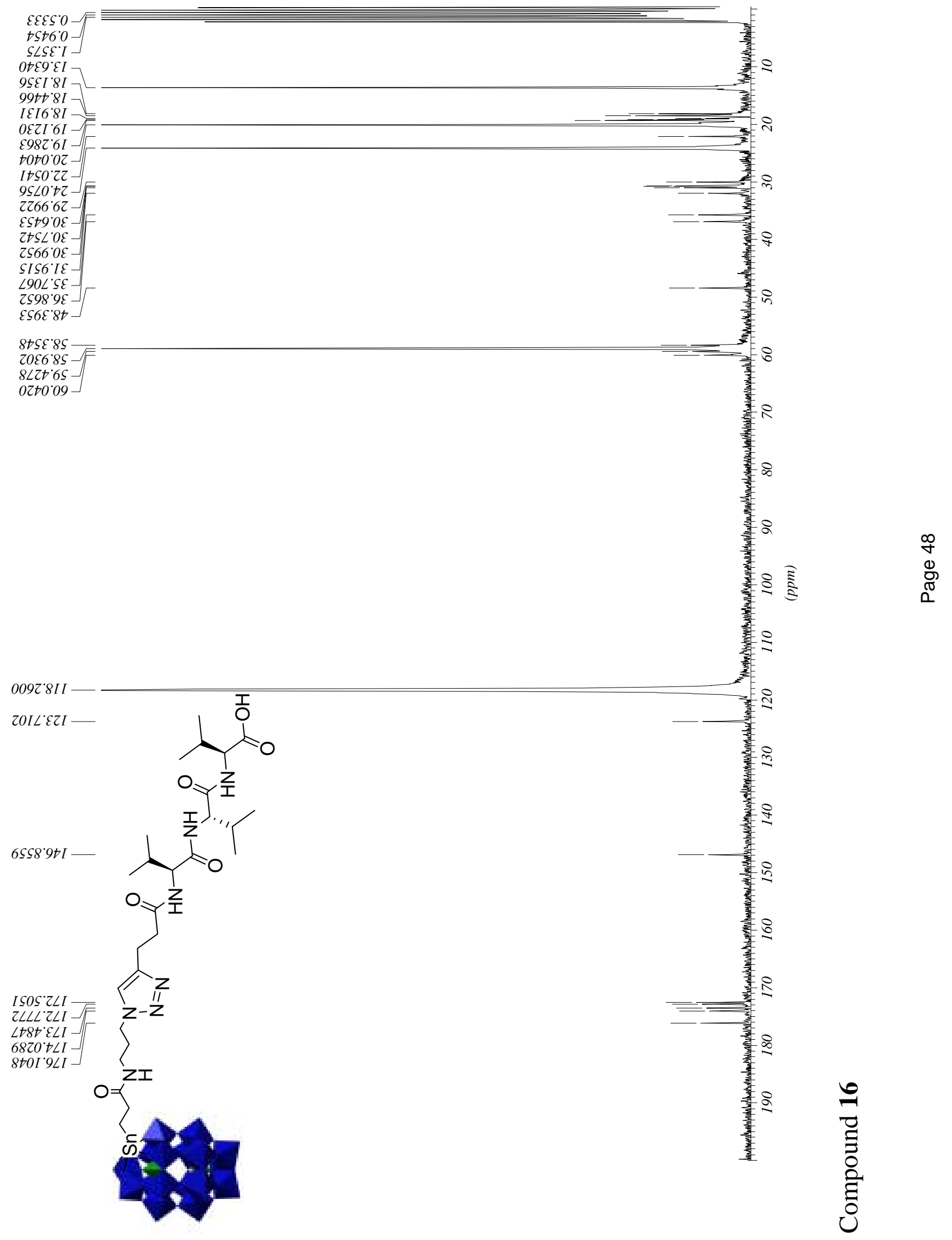




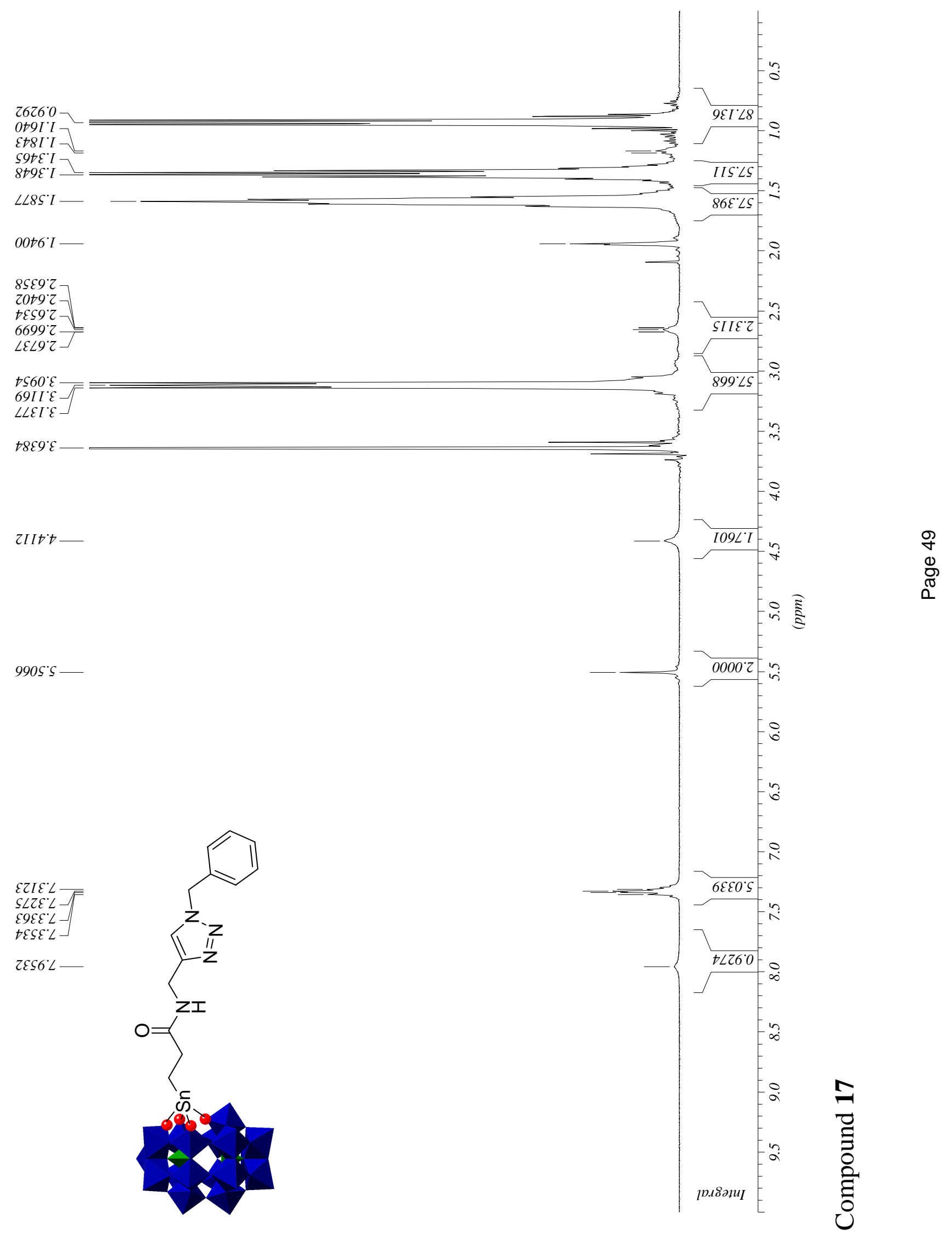



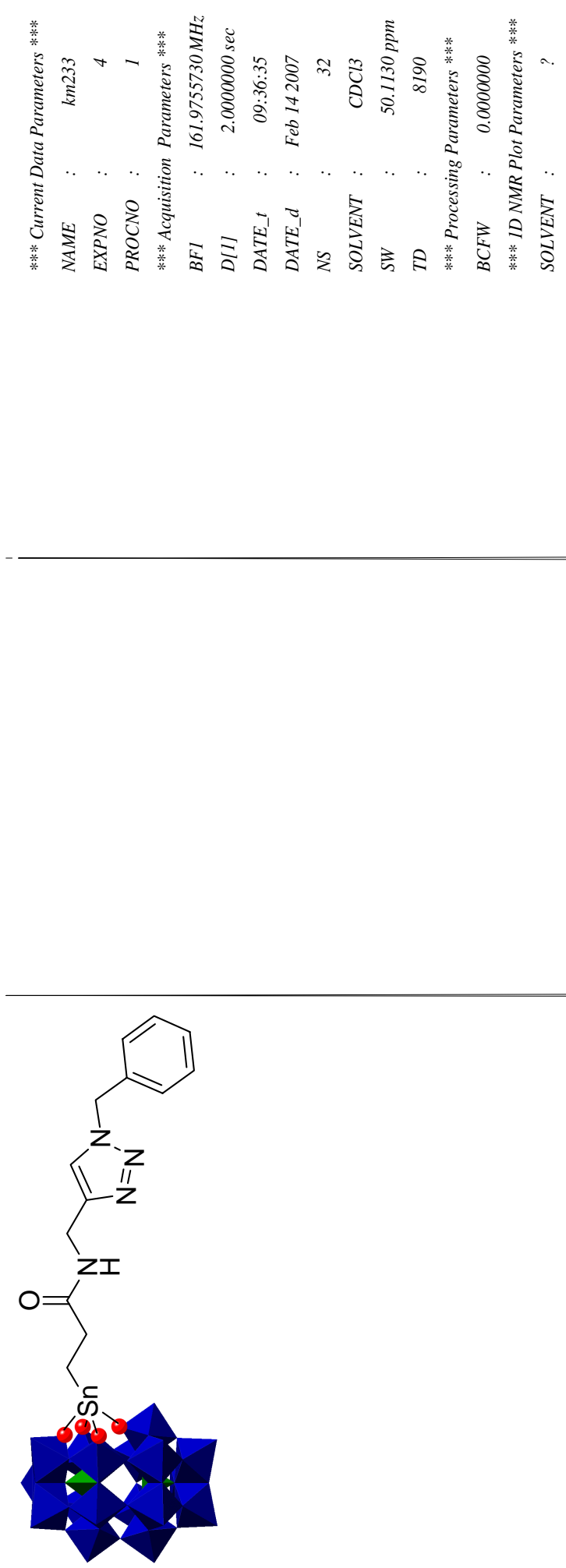


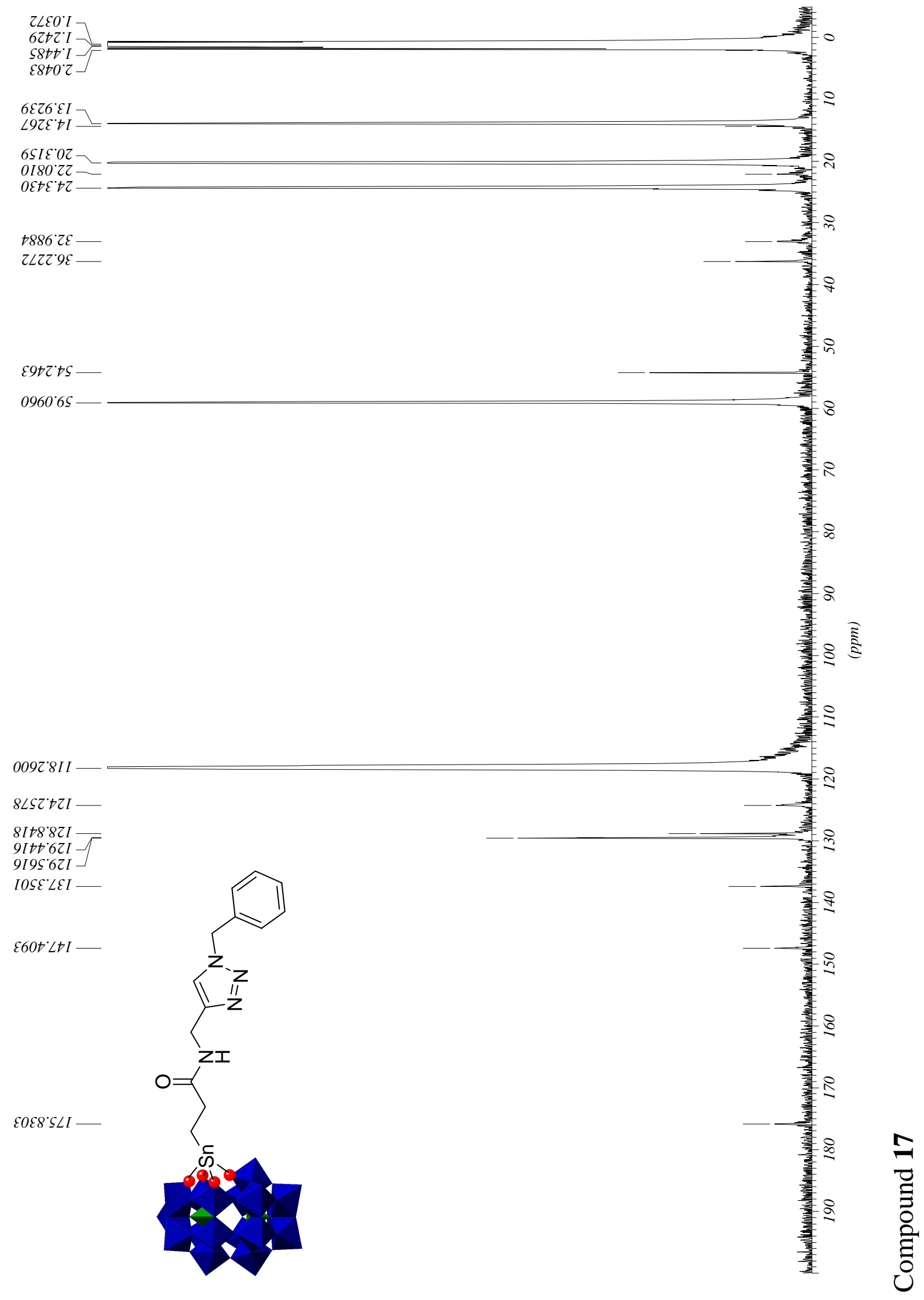




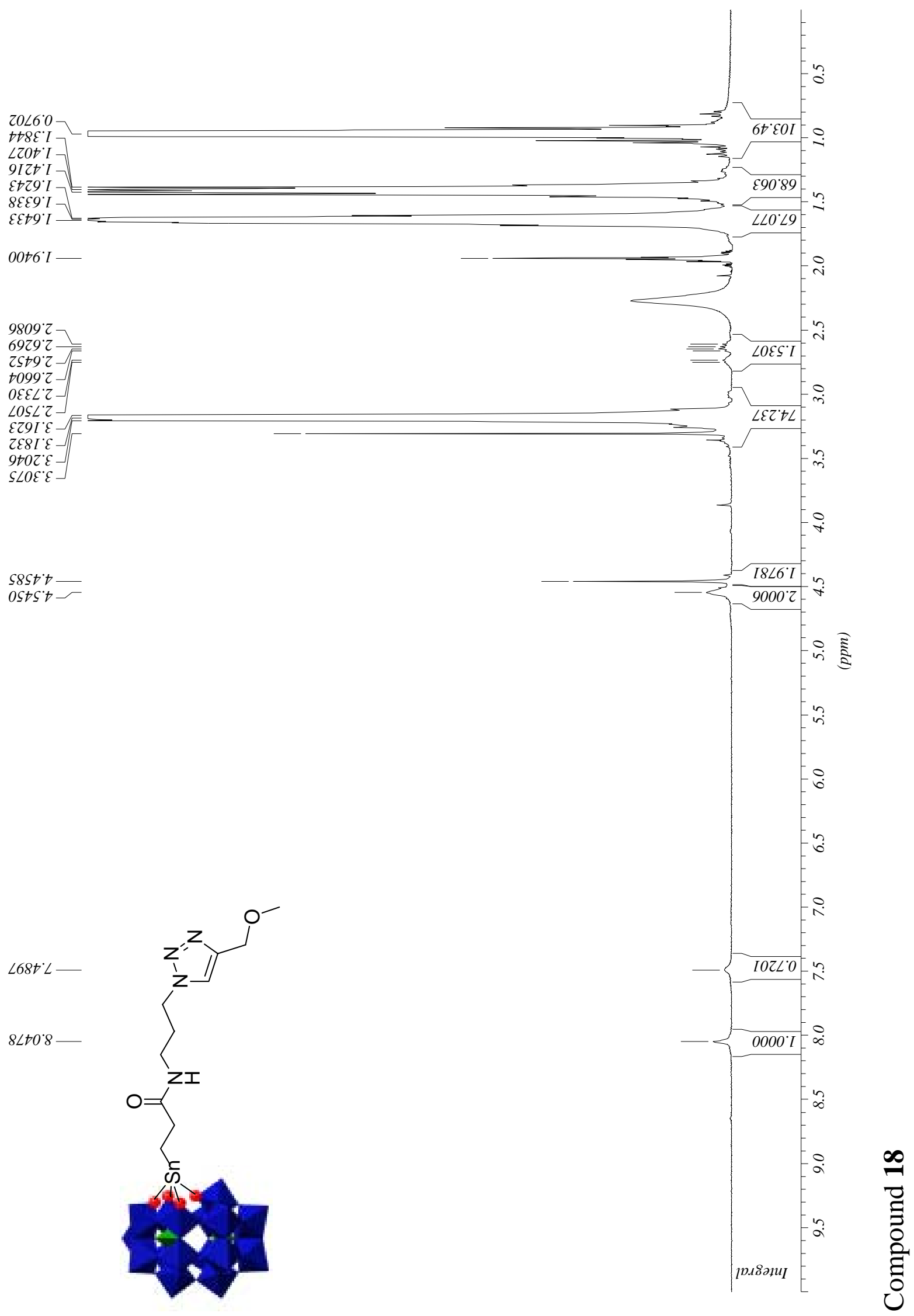




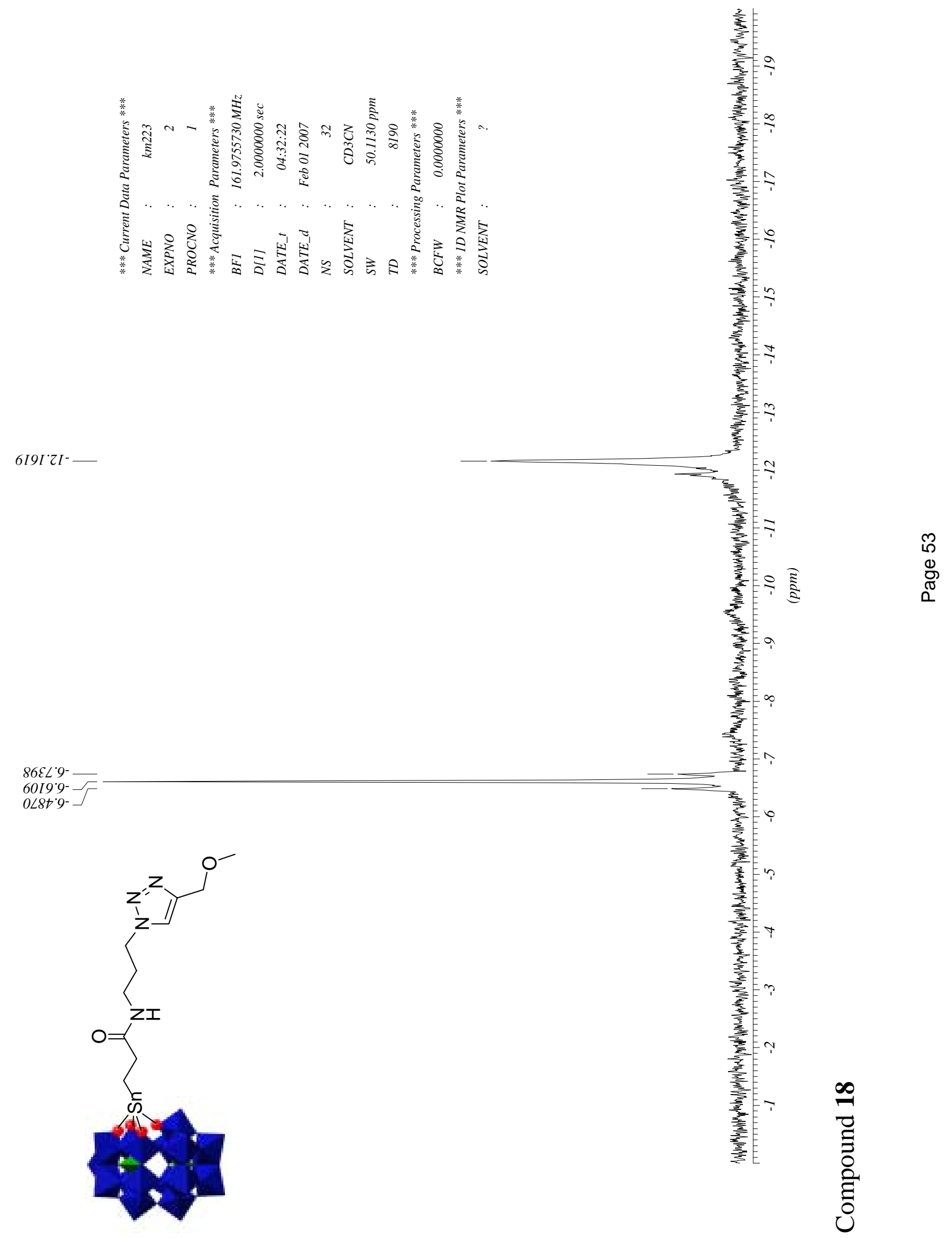




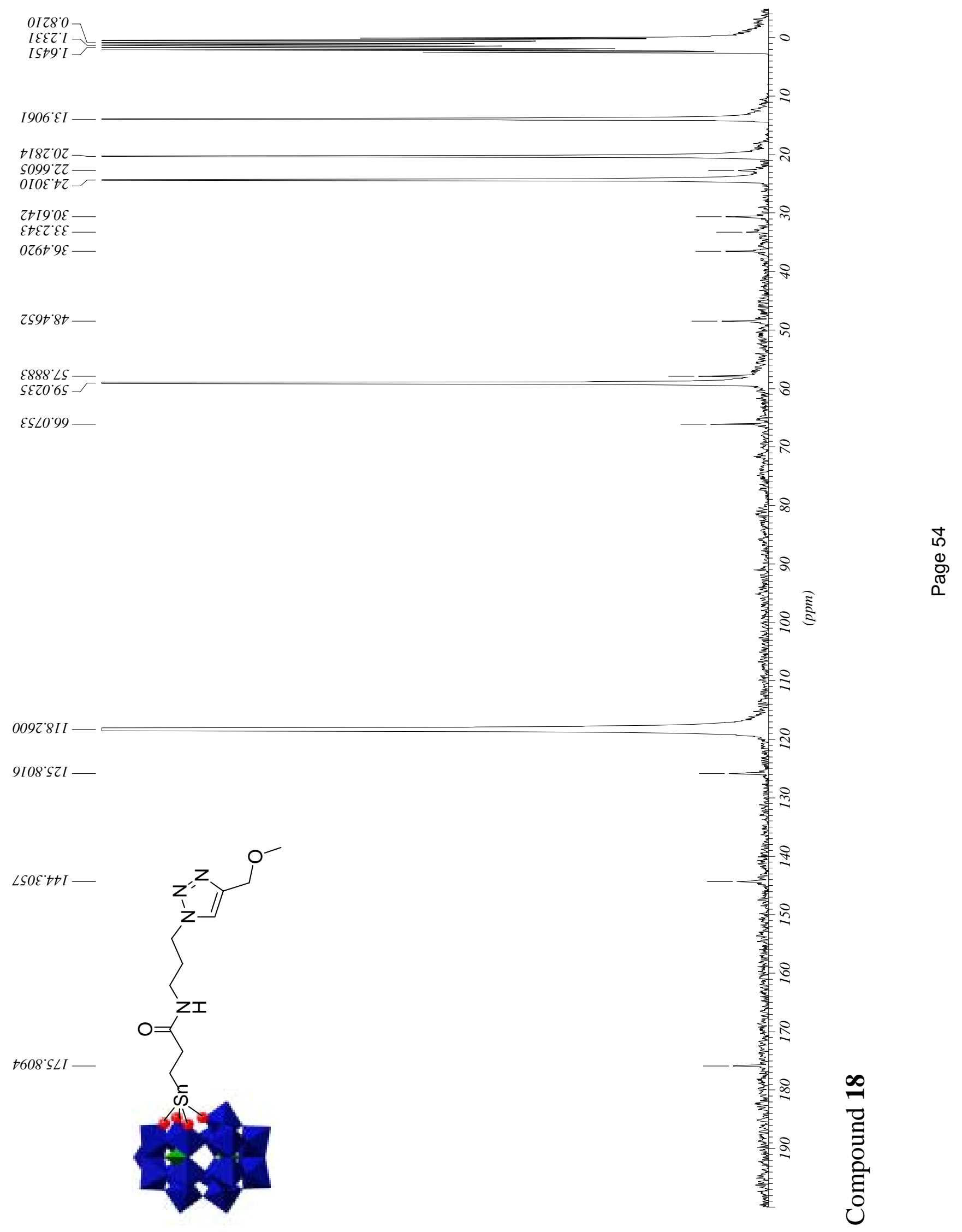




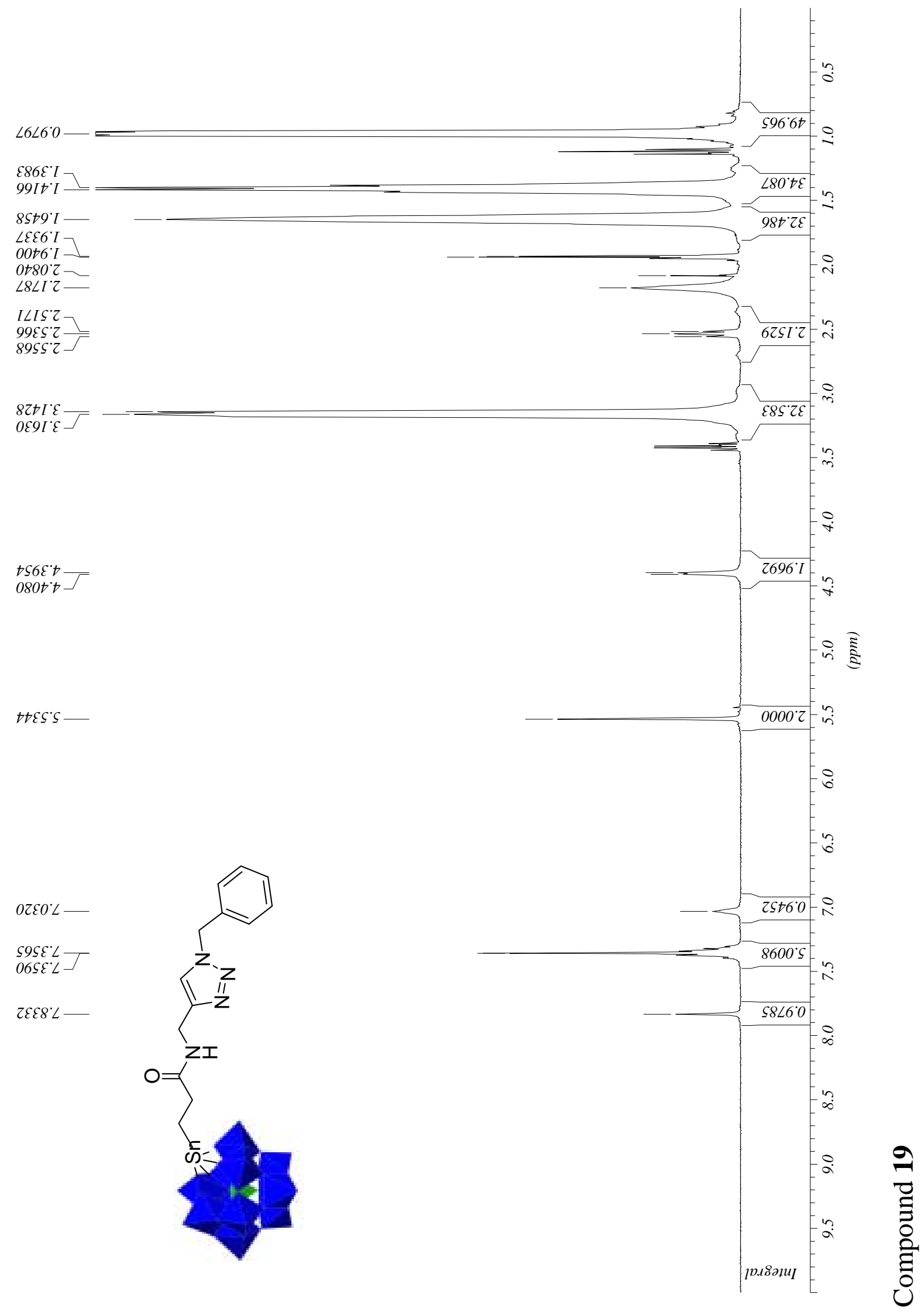




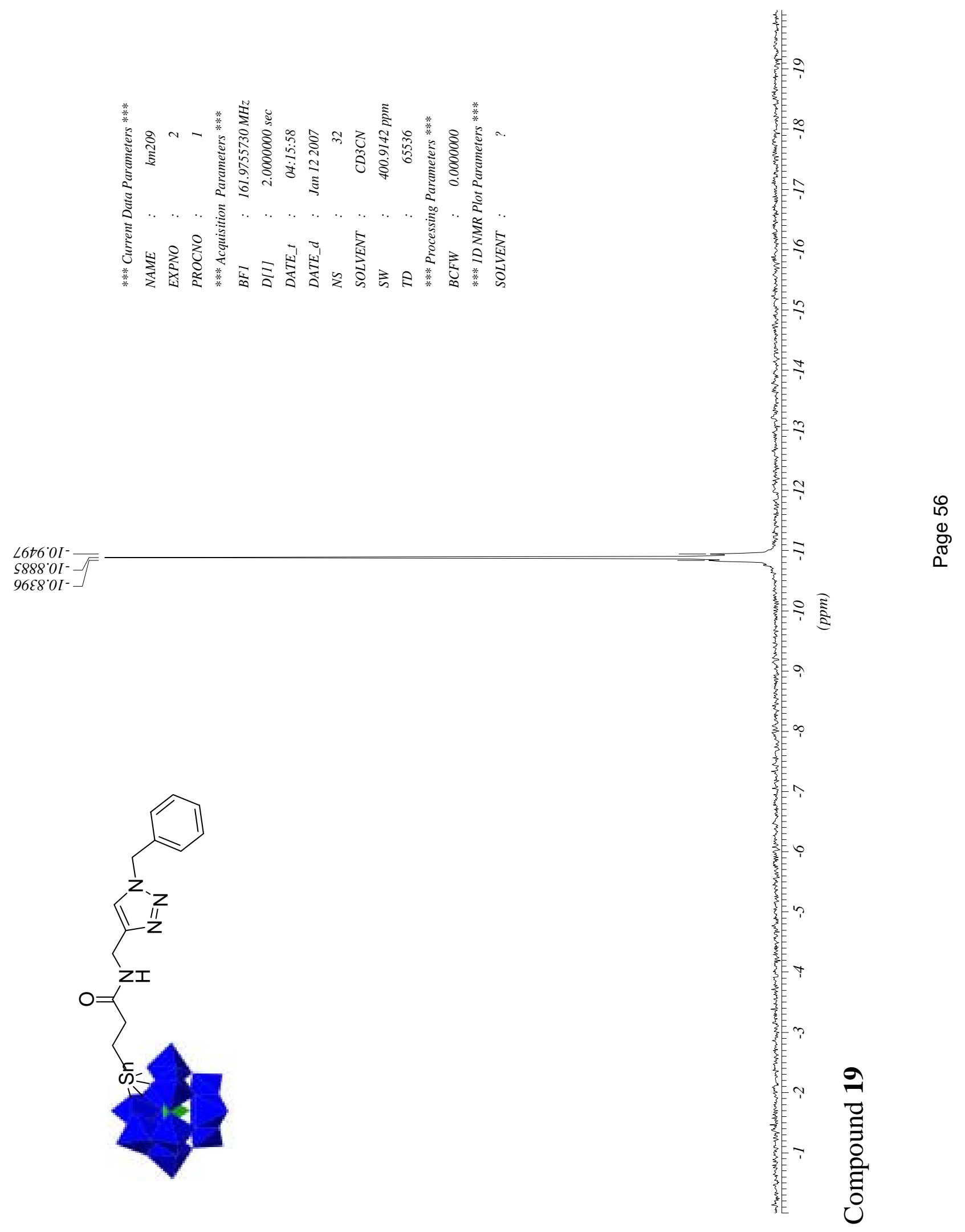




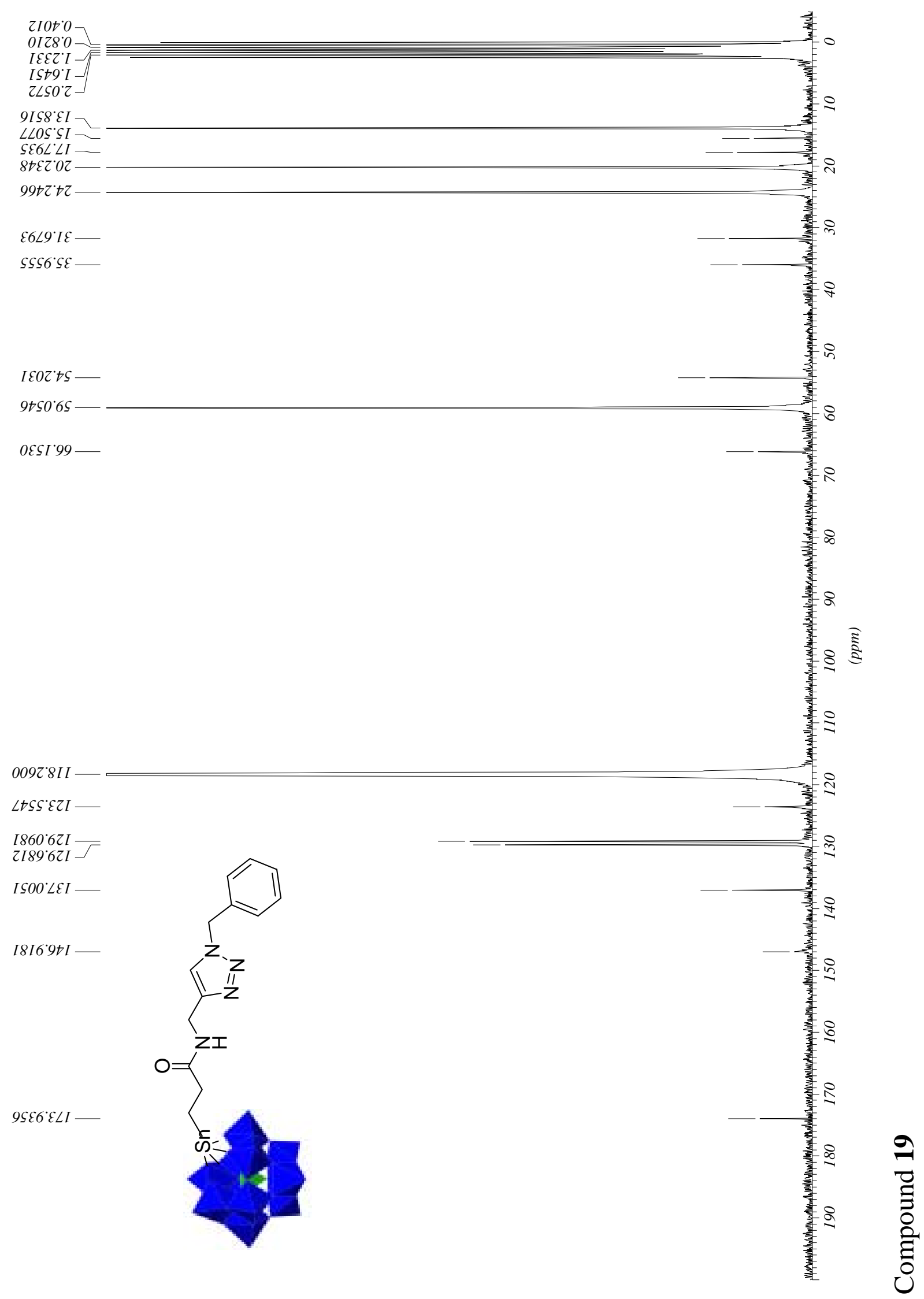




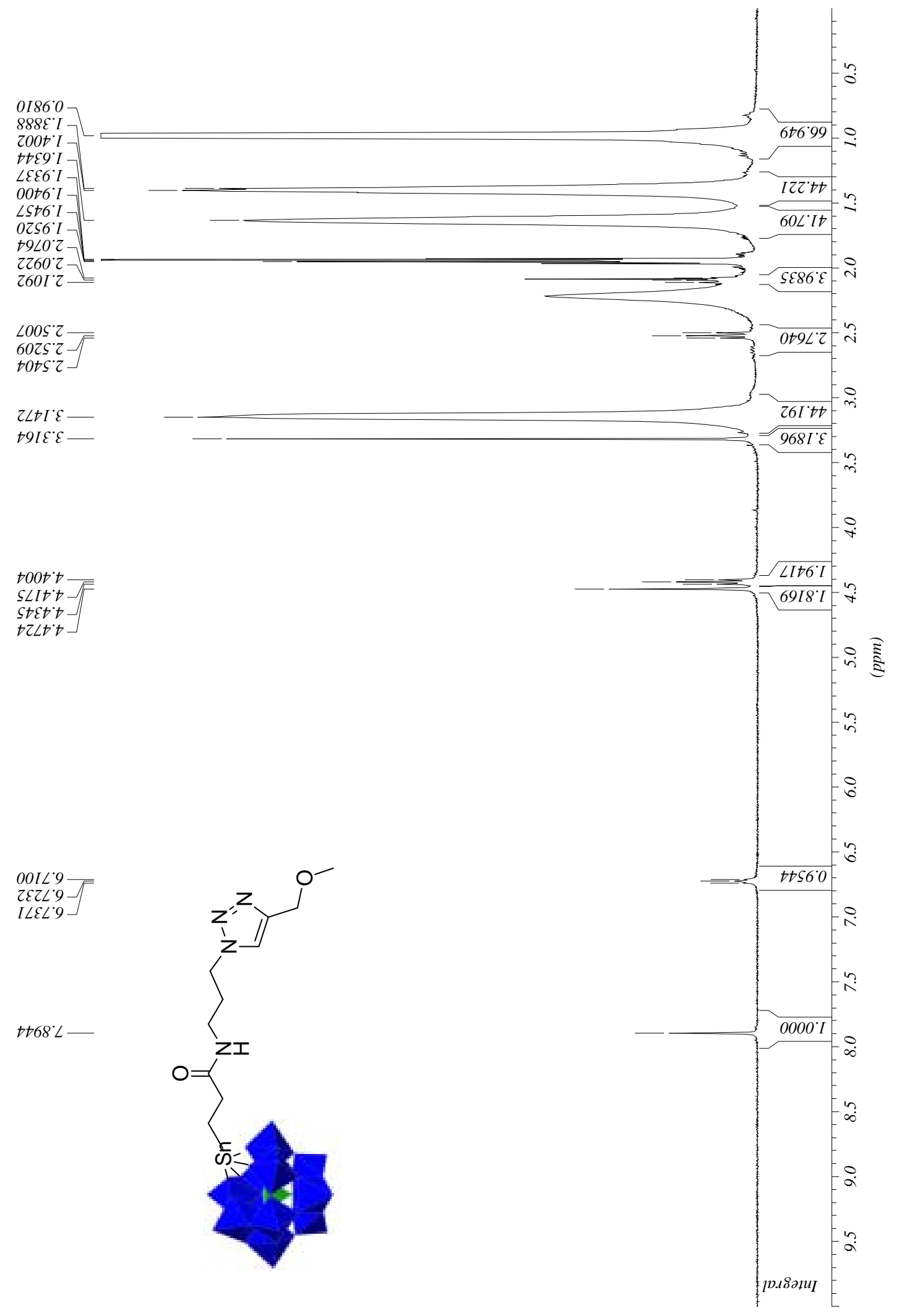




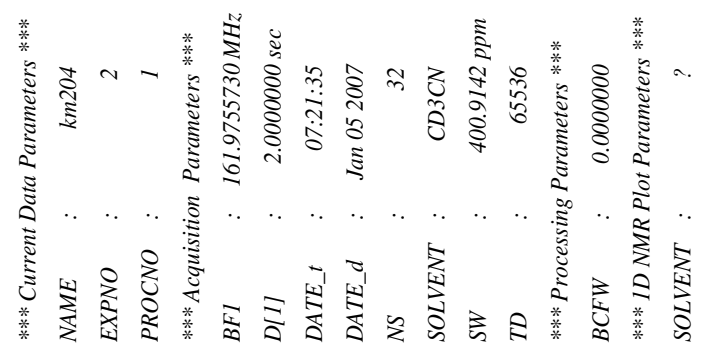

i
i
0
0
0

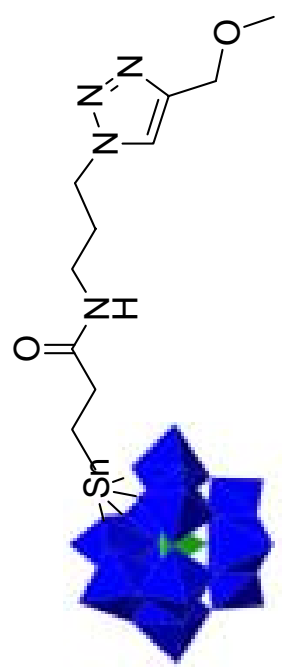

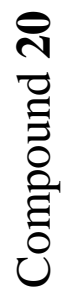




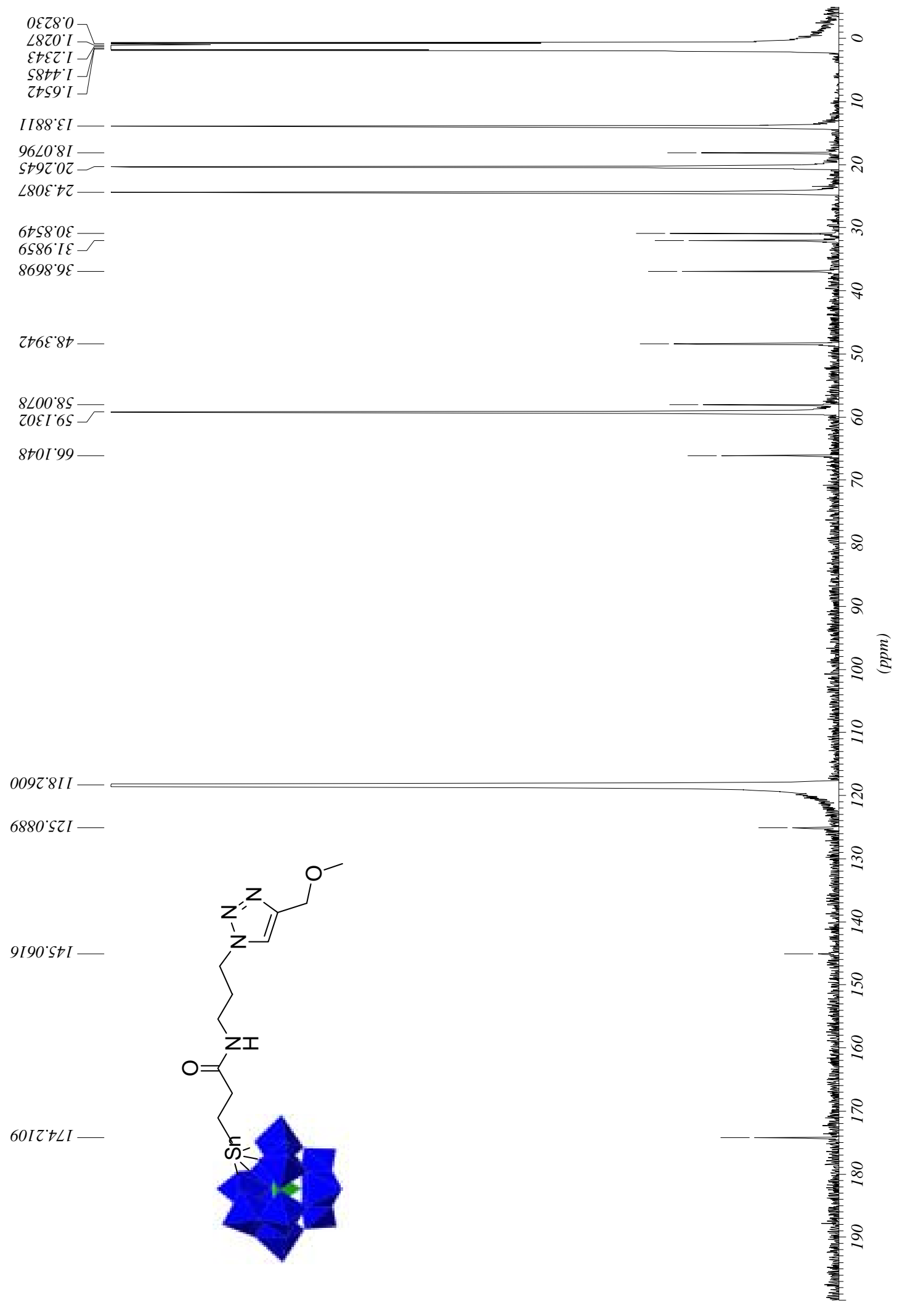




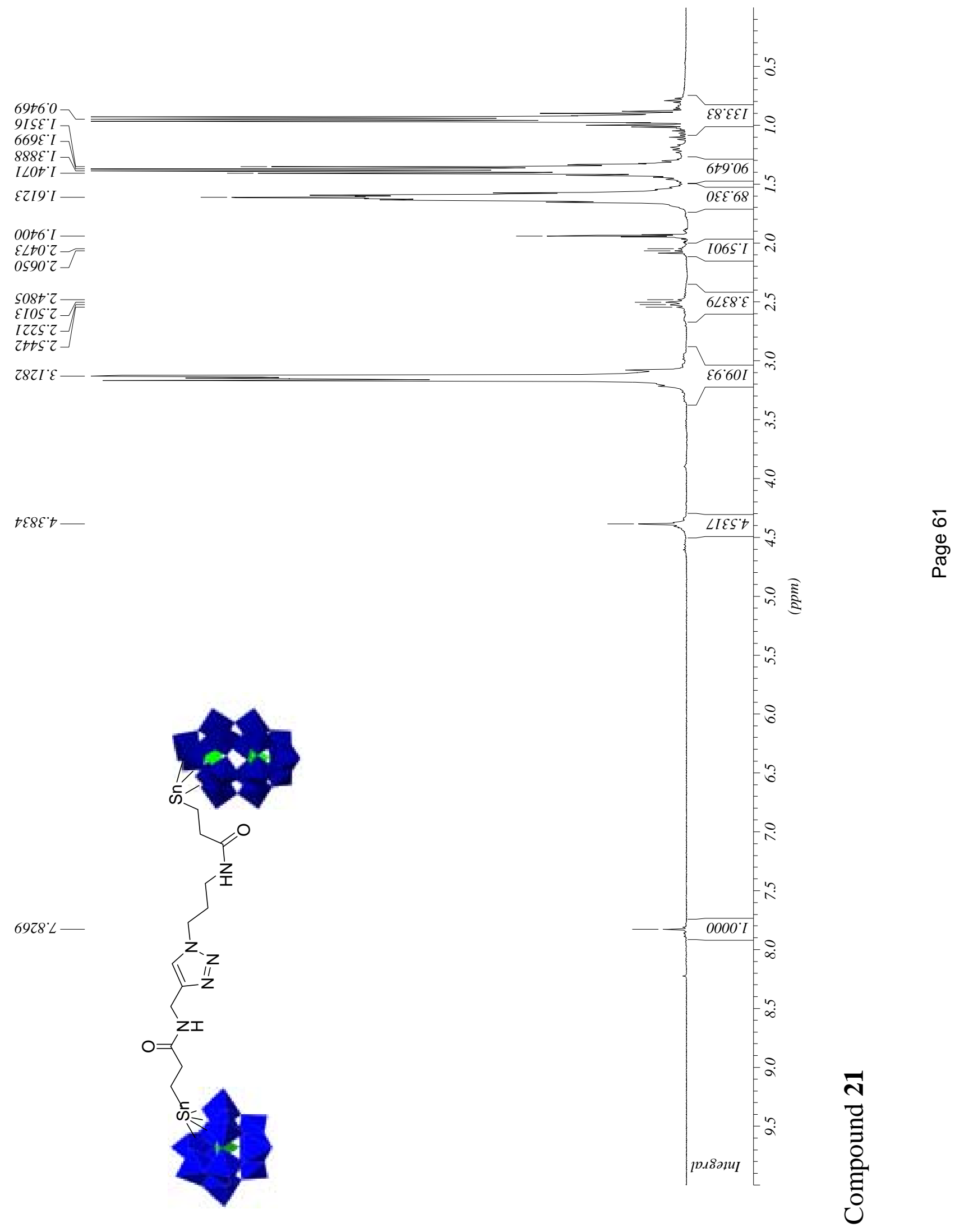




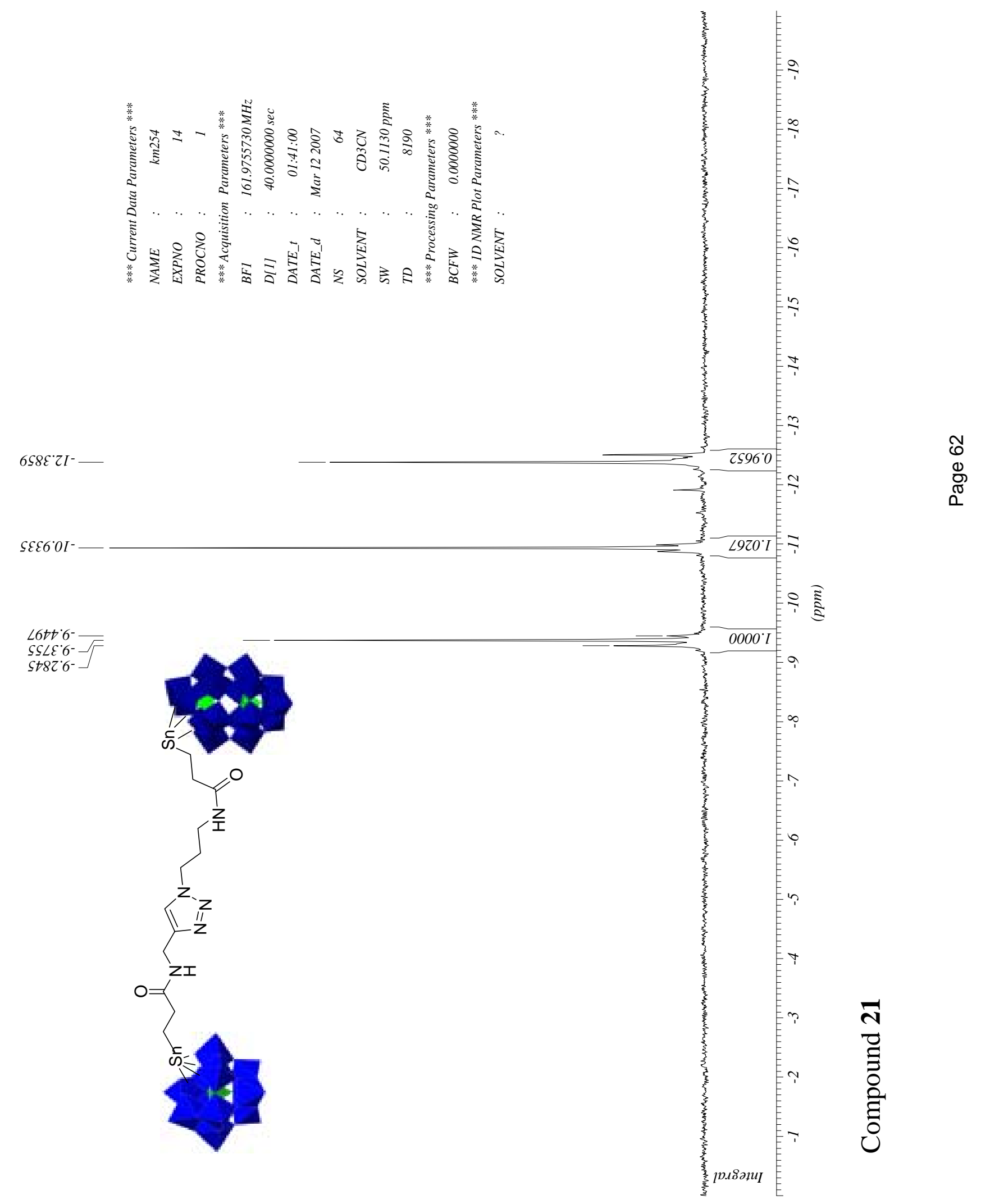




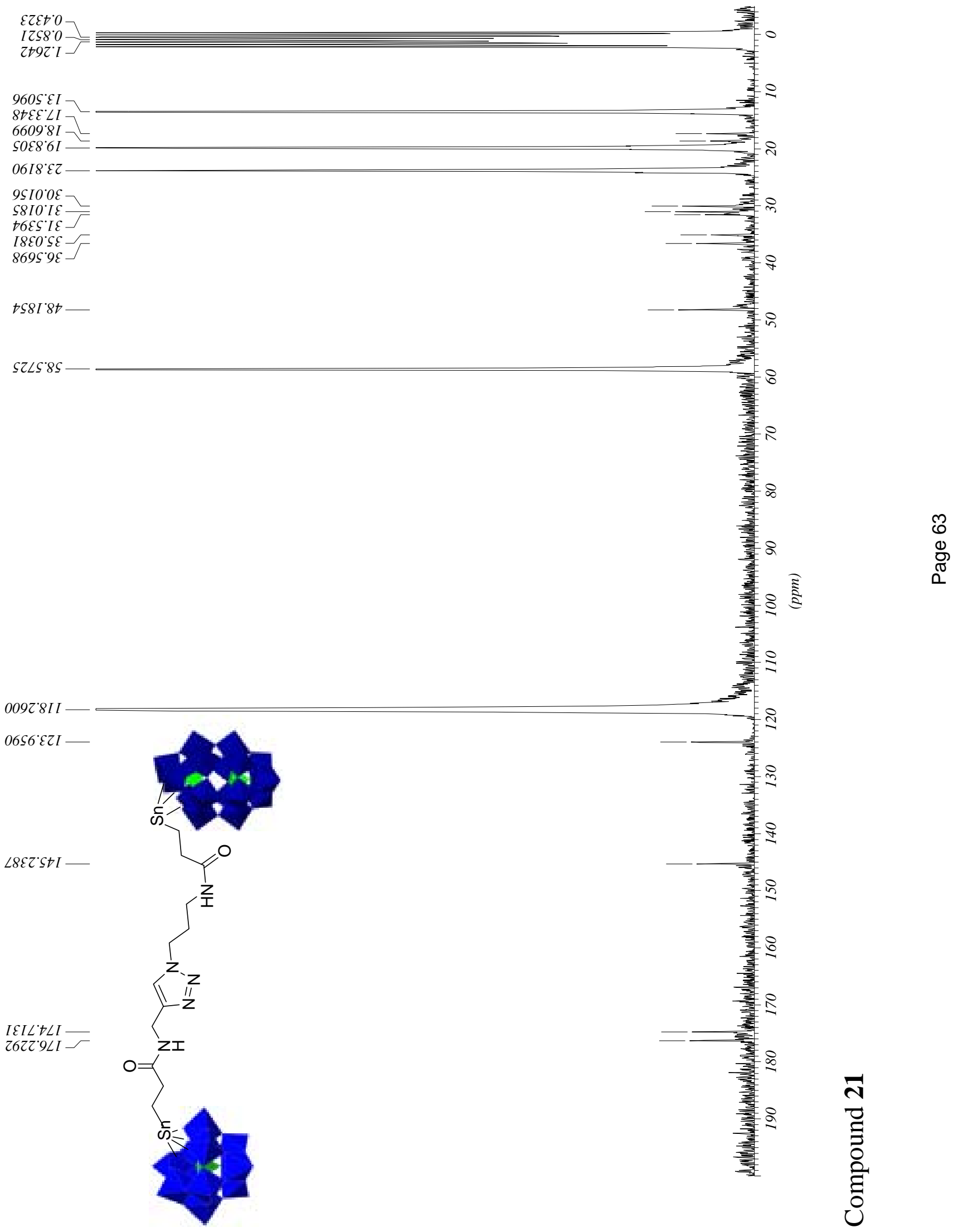

